$$
\begin{gathered}
\text { LN... } \\
\text { IN }-R
\end{gathered}
$$

\title{
An Artificial Compression Method For ENO Schemes

\author{
The Slope Modification Method
}

\author{
Huanan Yang* \\ Department of Mathematics, UCLA \\ Los Angeles, California 90024 \\ 8 september 1988
}

\begin{abstract}
A simple and efficient method of artificial compression is introduced. This method is based on a modification of the slopes of the ENO reconstruction and, with the help of suitably chosen parameters, greatly improves the resolution of the contact discontinuities. Numerical examples are provided to test the performance of the method and to give some suggestions as to the choice of the parameters.

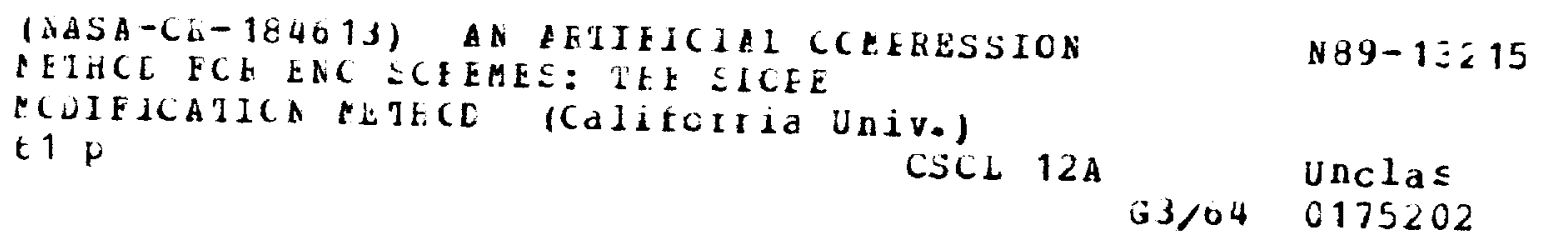

"Research partially supported by ONR Grant No. N00014- 86-K-0691, NSF Grant No. DMS 85-03294, NASA Langley Grant No. NAG1-270 and AFOSR Grant No. F49620-86-C-0115 


\title{
An Artificial Compression Method \\ For ENO Schemes
}

\section{The Slope Modification Method}

\author{
Huanan Yang \\ Department of Mathematics, UCLA \\ Los Angeles, California 90024
}

\section{$\S 1$ Introduction}

In this paper, we introduce a simple yet effective method to improve the performance of the ENO schemes for hyperbolic conservation laws.

Consider the following initial value problem:

$$
\left\{\begin{array}{l}
u_{t}+f(u)_{x}=0 \\
u(x, 0)=u_{0}(x)
\end{array}\right.
$$

where

$$
\begin{gathered}
u: \quad \mathbf{R}^{2} \rightarrow \mathbf{R}^{d} \\
f: \quad \mathbf{R}^{d} \rightarrow \mathbf{R}^{d}
\end{gathered}
$$

Assume that the system (1.1) is strictly hyperbolic in the sense that the Jacobian

$$
A=\frac{\partial f}{\partial u}
$$

has only real eigenvalues with a complete set of eigenvectors.

It is well known that the solution of (1.1) may develop discontinuities in finite time even though the initial value $u_{0}(x)$ is very smooth, say, a $C^{\infty}$ function. These discontinuities include shocks, contact discontinuities and the wave fronts of rarefaction waves.

It is natural that efforts in numerically simulating the solution of (1.1) with these structures mainly focus on designing numerical schemes with the following properties: 
i) achieving high accuracy in smooth regions of the solutions,

ii) producing sharp profiles for the shocks and the contact discontinuities,

iii) avoiding superfluous oscillations in front of or behind the shocks and the contact discontinuities,

iv) getting correct positions and speed of the discontinuities, and

v) avoiding non-physical discontinuities (e.g., expansion shocks).

The Lax-Wendroff theorem (see [13]) says that, for a convergent conservative scheme consistent with (1.1), the limit function of the numerical solution as the mesh size tends to zero is a weak solution of (1.1). Thus, conservative schemes automatically guarantees iv). Throughout this paper, we shall only consider schemes in conservation form.

To enforce v), one has to consider the so called entropy conditions which distinguish the physical solution from others. However, we are not going to discuss this problem here.

Recently, Harten, Osher, Engquist and Chakravarthy (see [2],[3] and [4]) introduced a class of essentially non-oscillatory (ENO) schemes which are of globally high order of accuracy where the solution is smooth. Although a complete theoretical analysis of these schemes has not been done, extensive numerical examples show that these schemes are nonlinearly stable. Hence, these schemes excellently meet the requirements i) and iii). For ii), the ENO schemes produce extremely sharp shock profiles. However, they smear the contact discontinuities at a rate which appears to be of order $O\left(n^{\frac{1}{r+1}}\right)$, where $r$ is the order of accuracy, and $n$ is the number of the time steps. In order to overcome this difficulty, Harten introduced the concept of subcell resolution. This led to excellent results in $1-d$ computation. He is currently considering 2- $d$ extensions. Another method which can be used to sharpen the contact discontinuity is Mao's method introduced in [11]. Merging his ideas with subcell resolution, Mao's method, hopefully, could be used with any known scheme. Some remarkable results have been obtained. However, much work needs to be done in order to make it practical in the cases of systems and of multi-dimensions.

In this paper we will introduce a procedure which is different from Harten's and Mao's and which greatly improves the performance of the ENO schemes at the contact discontinuities. This is going to be achieved by combining the ENO schemes with a $\operatorname{ACM}$ (artificial 
compression method, see Harten's pioneering work [1]) type technique. In this way, we can effectively prevent the contact discontinuities from being smeared and, in most cases, limit the transition of a numerically computed contact discontinuity to about 2 cells while essentially keeping the order of accuracy in the smooth regions of the solutions.

This procedure was originally invented for the cell average framework of the ENO schemes. Since then, Shu and Osher have transformed it to their pointwise ENO schemes. They have obtained surprisingly good results in 2-d computations. See [7] for the details.

In the next section, we will give a brief review of the ENO schemes. $\$ 3$ describes in detail our ACM type technique - the slope modification method and proves some of its properties. $\S 4$ applies the method to the system of Euler equations for gas dynamics. In $\S 5$, we will present some numerical results showing the performance of our method as well as giving some suggestions as to the choice of the parameters concerned.

\section{\$2 Review of the ENO schemes}

We refer to [2], [3], [4], [5], [6], [7] and [8] for details of the cell average ENO schemes and their pointwise versions. Here we only give a very brief review of the schemes. This is necessary for us to describe our slope modifying method.

Originally, the ENO schemes were introduced for cell average values of the solutions as high order extensions of the Godunov scheme and the MUSCL scheme. Denote by $v$ the numerical solution approximating the sliding average $\bar{u}$ of the exact solution $u$ of (1.1), i.e.,

$$
\begin{aligned}
v(x, t) & \doteq \bar{u}(x, t) \\
& =\frac{1}{h} \int_{x-\frac{1}{2} h}^{x+\frac{1}{2} h} u(y, t) d y .
\end{aligned}
$$

Let $\left\{\left[x_{j-\frac{1}{2}}, x_{j+\frac{1}{2}}\right] \times\left[t_{n}, t_{n+1}\right]\right\}$ where $x_{\alpha}=\alpha h, t_{n}=n \tau, j=0, \pm 1, \pm 2, \cdots, n=0,1,2, \cdots$, be a partition of $\mathbf{R} \times \mathbf{R}^{+}$. Denote $u\left(x_{j}, t_{n}\right)$ by $u_{j}^{n}$ and $v\left(x_{j}, t_{n}\right)$ by $v_{j}^{n}$. Assume that $E(t)$ is the exact solution operator of (1.1). I.e., if $u(x, t)$ is the solution of $(1.1)$, then

$$
u(x, t)=E(t) u(x, 0) .
$$


The theoretical ENO scheme can be written as

$$
v_{j}^{n+1}=\frac{1}{h} \int_{x_{j-\frac{1}{2}}}^{x_{j+\frac{1}{2}}} E(\tau) R\left(x, v^{n}\right) d x
$$

which is said to be 'theoretical' since $E(t)$ is exact. In $(2.1), R\left(x, v^{n}\right)$ is a pointwise approximation of the solution $u$ at time $t^{n}$ derived from the cell average approximate solution $v^{n}$. It is a piecewise polynomial. In fact, it is a polynomial in each cell $\left(x_{j-\frac{1}{2}}, x_{j+\frac{1}{2}}\right)$. The procedure of computing $R\left(x, v^{n}\right)$ from $v^{n}$ is called reconstruction.

Given the cell averages $\left\{v_{j}\right\}_{j=-\infty}^{\infty}$ of a function $u(x)$, in [3] two ways to compute $R\left(x, v^{n}\right)$, reconstruction via primitive function $(R P)$ and reconstruction via deconvolution (RD) were described. We only review RP here since a knowledge of it is enough for us to introduce our slope modification method.

Notice that, for any fixed integer $i_{0},\left\{p_{i}\right\}_{i=i_{0}}^{\infty}=\left\{\sum_{j=i_{0}}^{i} v_{j} h\right\}_{i=i_{0}}^{\infty}$ is a sequence of pointwise values of the primitive function $p(x)=\int_{x_{i_{0}-\frac{1}{2}}}^{x} u(x) d x$ of $u(x)$ at $x_{i_{0}-\frac{1}{2}}, x_{i_{0}+\frac{1}{2}}, x_{i_{0}+\frac{3}{2}}, \ldots$. A natural way of getting a polynomial which approximates $u$ in the cell $\left(x_{j-\frac{1}{2}}, x_{j+\frac{1}{2}}\right)$ is, therefore, to interpolate $p(x)$ at $r+1$ consequent points $x_{i(j)-\frac{1}{2}}, x_{i(j)+\frac{1}{2}}, \ldots, x_{i(j)+r+\frac{1}{2}}$, including $x_{j-\frac{1}{2}}$ and $x_{j+\frac{1}{2}}$. The derivatives of the interpolating polynomial then give the reconstruction and its derivatives. One has $\mathrm{r}$ degrees of freedom of choosing $i(j)$, i.e., the stencil. It is the way of choosing the stencil that distinguishes the ENO schemes from others.

For simplicity, we only give the hierarchical algorithm for evaluating $i(j)$. The goal is to find the "smoothest" stencil which includes $x_{j-\frac{1}{2}}$ and $x_{j+\frac{1}{2}}$. The smoothness of the stencil is somehow measured by the absolute value of the divided differences $p\left[x_{j}, x_{j+1}, \cdots, x_{j+k}\right]$ which are defined inductively by

$$
p\left[x_{j}\right]=p\left(x_{j}\right) \quad j=-\infty, \cdots,+\infty,
$$

and

$$
\begin{aligned}
p\left[x_{j}, x_{j+1}, \cdots, x_{j+k}\right]= & \left(p\left[x_{j+1}, \cdots, x_{j+k}\right]-p\left[x_{j}, \cdots, x_{j+k-1}\right] /\left(x_{j+k}-x_{j}\right)\right. \\
k & =1,2, \cdots, r \\
i & =-\infty, \cdots, \infty
\end{aligned}
$$

We describe the algorithm evaluating $i(j)$ in the flavor of Fortran language as follows: 
1) $i(j)=j$.

2) For $k=1,2, \cdots, r$, if

$$
\left|p\left[x_{i(j)-1-\frac{1}{2}}, \cdots, x_{i(j)-1-\frac{1}{2}+k}\right]\right| \leq\left|p\left[x_{i(j)-\frac{1}{2}}, \cdots, x_{i(j)-\frac{1}{2}+k}\right]\right|
$$

then

$$
i(j)=i(j)-1
$$

Having computed $i(j)$, one then gets a polynomial $p_{j}(x, v)$ by Newton's form of interpolation. From this the reconstruction $R(x, v)$ and its derivatives at $x=x_{j}$ are given by

$$
\left.\frac{d^{l}}{d x^{l}} R(x, v)\right|_{x=x_{j}}=\left.\frac{d^{l+1}}{d x^{l+1}} p_{j}(x, v)\right|_{x=x_{j}}, \quad l=0,1, \cdots, r-1 .
$$

\section{§3 The Slope Modification Method for Scalar Conservation Laws}

Denote by $\delta_{j-\frac{1}{2}}$ the jump of $R(x, v)$ at the cell interface $x_{j-\frac{1}{2}}$, i.e.,

$$
\delta_{j-\frac{1}{2}}=R\left(x_{j-\frac{1}{2}}+0, v\right)-R\left(x_{j-\frac{1}{2}}-0, v\right) .
$$

We introduce the following slope modification algorithm:

Algorithm 3.1. The modified reconstruction $\hat{R}(x, v)$ in $\left(x_{j-\frac{1}{2}}, x_{j+\frac{1}{2}}\right)$ is a $(r-1)$-th order polynomial which is defined by

$$
\begin{gathered}
\left.\frac{d^{l}}{d x^{l}} \hat{R}(x, v)\right|_{x=x_{j}}=\left.\frac{d^{l}}{d x^{l}} R(x, v)\right|_{x=x_{j}}, \quad l \leq r-1, l \neq 1, \\
\left.\frac{d}{d x} \hat{R}(x, v)\right|_{x=x_{j}}=\left.\frac{d}{d x} R(x, v)\right|_{x=x_{j}}+\partial S_{j} / h,
\end{gathered}
$$

where the slope modifier $\partial S_{j}$ is given by

$$
\partial S_{j}=2 \mathrm{~m}\left(\alpha_{j} \mathrm{~m}\left(\delta_{j-\frac{1}{2}}, \delta_{j+\frac{1}{2}}\right), \mathrm{m}\left(v_{j+1}-R_{j+\frac{1}{2}}^{-}, R_{j-\frac{1}{2}}^{+}-v_{j-1}\right)\right),
$$

here $\left\{\alpha_{j}\right\}$ is a sequence of positive numbers, the function $\mathrm{m}(x, y)$ is defined by

$$
\mathrm{m}(x, y)= \begin{cases}0, & x y \leq 0, \\ \min (|x|,|y|) \operatorname{sgn}(x), & \text { otherwise, }\end{cases}
$$


and $R_{j+\frac{1}{2}}^{ \pm}$denotes $R\left(x_{j+\frac{1}{2}} \pm 0, v\right)$.

To see the effect of the algorithm, let us apply it to the UNO scheme introduced in [1]. The UNO scheme is based on a non-oscillatory piecewise linear reconstruction. More precisely, if $v=\left\{v_{j}\right\}_{j=-\infty}^{\infty}$ are the cell averages of a function $u$, the UNO reconstruction $R(x, v)$ can be written as

$$
R(x, v)=v_{j}+S_{j} \frac{x-x_{j}}{h}, \quad x_{j-\frac{1}{2}}<x<x_{j+\frac{1}{2}} .
$$

The reconstruction is non-oscillatory in the sense that the number of the extrema of $R(\cdot, v)$ is no more than that of $v$. We recall that $R(\cdot, v)$ satisfies(see [2])

$$
\begin{gathered}
\delta_{j-\frac{1}{2}}\left(v_{j}-v_{j-1}\right) \geq 0 \\
\delta_{j-\frac{1}{2}}=0, \quad \text { if } v_{j}-v_{j-1}=0, \\
S_{j} \mathrm{~m}\left(v_{j+1}-v_{j}, v_{j}-v_{j-1}\right) \geq 0 .
\end{gathered}
$$

Recall also that (see [1]), if the numerical scheme for scalar conservation law (1.1) is

$$
v_{j}^{n+1}=v_{j}^{n}-\lambda\left(\hat{f}_{j+\frac{1}{2}}-\hat{f}_{j-\frac{1}{2}}\right)
$$

a modified scheme adding artificial compression to (3.10) would be

$$
v_{j}^{n+1}=v_{j}^{n}-\lambda\left(\bar{f}_{j+\frac{1}{2}}-\bar{f}_{j-\frac{1}{2}}\right)
$$

where the flux modifier

$$
g_{j+\frac{1}{2}}=\bar{f}_{j+\frac{1}{2}}-\hat{f}_{j+\frac{1}{2}}
$$

obeys

$$
g_{j+\frac{1}{2}} \triangle u_{j}^{n} \geq 0
$$

Consider the UNO scheme with numerical flux(see [2])

$$
\hat{f}_{j+\frac{1}{2}}= \begin{cases}f\left(v_{j}^{n}\right)+0.5 \bar{a}_{j+\frac{1}{2}}\left(1-\lambda \bar{a}_{j-\frac{1}{2}}\right) S_{j}^{n} /\left[1+\lambda\left(\bar{a}_{j+\frac{1}{2}}-\bar{a}_{j-\frac{1}{2}}\right)\right] & \text { if } \bar{a}_{j+\frac{1}{2}} \geq 0 \\ f\left(v_{j+1}^{n}\right)-0.5 \bar{a}_{j+\frac{1}{2}}\left(1+\lambda \bar{a}_{j+\frac{3}{2}}\right) S_{j+1}^{n} /\left[1+\lambda\left(\bar{a}_{j+\frac{3}{2}}-\bar{a}_{j+\frac{1}{2}}\right)\right] & \text { if } \bar{a}_{j+\frac{1}{2}}<0\end{cases}
$$

where

$$
\bar{a}_{j+\frac{1}{2}}=\left(f\left(v_{j+1}^{n}\right)-f\left(v_{j}^{n}\right)\right) /\left(v_{j+1}^{n}-v_{j}^{n}\right)
$$


In the remaining part of this section, by ' the UNO scheme', we always mean the scheme with this flux.

Applying the slope modification method to the UNO scheme, one gets a scheme whose numerical flux has the same expression as (3.14) except that $S_{j}$ is replaced by $\hat{S}_{j}$ for all $j$.

From (3.4), (3.7), (3.8) and (3.13) one can see that under the CFL type conditions

$$
\left|\lambda \bar{a}_{j+\frac{1}{2}}\right|<1
$$

and

$$
\lambda\left(\bar{a}_{j+\frac{1}{2}}-\bar{a}_{j-\frac{1}{2}}\right)>-1,
$$

algorithm 3.1. applied to the UNO scheme has the effect of adding artificial compression to the UNO scheme.

Remark 3.2. For general high order ENO schemes, (3.7), (3.8) and (3.9) are not necessarily true. Nevertheless, the numerical experiments in [4] (see the figures of the reconstructions at the end of [4]) show that they are essentially true. Furthermore, the numerical results we are going to report in $\S 5$. show that the slope modifier with suitable chosen $\alpha_{j}$ works well for all the cases that we have tested, although a rigorous analysis is not available now.

Next, we discuss the stability of our method. Since the modified reconstruction no longer obeys (3.7), (3.8) and (3.9), the algorithm does introduce oscillations to the reconstruction. However, due to the cell average process, it seldom introduces oscillations to the solution at the next time level. In fact, we have

Theorem 3.3. If $\lambda\left|\bar{a}_{j+\frac{1}{2}}-\bar{a}_{j-\frac{1}{2}}\right|<1$ and $\lambda\left|\bar{a}_{j+\frac{1}{2}}\right|<1$ hold for all $j$, then the modified scheme obtained by applying algorithm 3.1. to the UNO scheme is non-oscillatory.

In order to prove the theorem, we first describe the procedure for deriving the modified UNO scheme. This procedure is a direct copy of that for the UNO scheme. Having obtained the reconstruction $R\left(x, v^{n}\right)$ and the corresponding modified reconstruction $\hat{R}\left(x, v^{n}\right)$, one derive pointwise approximate solutions $w(x)$ and $\hat{w}(x)$ at time $t_{n+1}$ as follows: both $w(x)$ and $\hat{w}(x)$ are piecewise linear with possible discontinuities at

$$
X_{j+\frac{1}{2}}=x_{j+\frac{1}{2}}+\bar{a}_{j+\frac{1}{2}} \tau
$$


such that

$$
w\left(X_{j+\frac{1}{2}} \pm 0\right)=R\left(x_{j+\frac{1}{2}} \pm 0, v^{n}\right)
$$

and

$$
\hat{w}\left(X_{j+\frac{1}{2}} \pm 0\right)=\hat{R}\left(x_{j+\frac{1}{2}} \pm 0, v^{n}\right) .
$$

We shall call these points of possible discontinuities $d$-points. The modified UNO scheme is then completed by defining

$$
v_{j}^{n+1}=\frac{1}{h} \int_{x_{j-\frac{1}{2}}}^{x_{j+\frac{1}{2}}} \hat{w}(x) d \dot{x} .
$$

For convenience, let us make some definitions and notations.

Definition 3.4. A sequence of points $x_{i}, x_{i+1}, \ldots, x_{j}$ is called a strict maximum section of a cell average function $v=\left\{v_{j}\right\}_{j=-\infty}^{\infty}$ and is denoted by $\bar{S}(v ; i, j)$ if

$$
v_{i-1}<v_{i}=v_{i+1}=\cdots=v_{j}>v_{j+1}
$$

Denote by $\bar{S}(v)$ the set of all such $\bar{S}(v ; i, j)$ 's.

Definition 3.5. An interval $[a, b], a \leq b$ is called a strict maximum section of a piecewise linear function $u$ and is denoted by $\bar{s}(u ; a, b)$ if there exists a $\delta>0$ such that

$$
u(x)<c=\lim _{\xi \rightarrow a} \sup u(\xi)
$$

holds for $a-\delta<x<a$ or $b<x<b+\delta$, and such that $u(x)=c$ if $a<x<b$. Denote by $\bar{s}(u)$ the set of all such $\bar{s}(u ; a, b)$ 's.

Similarly, one can define strict minimum sections $\underline{S}(v ; i, j)$ and $\underline{s}(u ; a, b)$ along with corresponding sets $\underline{S}(v)$ and $\underline{s}(u)$.

Remark 3.6. In the Definition 3.5., if $u$ is the UNO reconstruction of a cell average function $v$, and if $a=b$, then one can make $u(x)$ continuous at $x=a$ by defining $u(a)=c$. The same observation holds for the strict minimum sections.

Remark 3.7. If $\bar{s}(w ; a, b)$ is a strict maximum section of $w$, then $\bar{s}(\hat{w} ; a, b)$ is also a strict maximum section of $\hat{w}$. Furthermore,

$$
\lim _{\xi \rightarrow a} \sup \hat{w}(\xi)=\lim _{\xi \rightarrow a} \sup w(\xi)
$$

The same observation holds for the strict minimum sections. 
We denote by $\bar{N}(u)$ the number of the strict maximum sections of a function $u$, by $\underline{N}(u)$ the number of its strict minimum sections and by $N_{e}(u)$ the number of all its strict extremum sections. Finally, we denote by $u[a, b]$ the average of a pointwise function $u$ over $(a, b)$.

We have trivially,

$$
N_{e}(w) \leq N_{e}\left(R\left(\cdot, v^{n}\right)\right)
$$

We will prove the theorem by showing that

$$
N_{e}\left(v^{n+1}\right) \leq N_{e}(w)
$$

This inequality is implied by the following two inequalities

$$
\bar{N}\left(v^{n+1}\right) \leq \bar{N}(w)
$$

and

$$
\underline{N}\left(v^{n+1}\right) \leq \underline{N}(w)
$$

Since the proofs of the two inequalities are same, it suffices to prove (3.25) which is equivalent to the following

Claim. There is a 1-1 mapping

$$
\mathrm{P}: \bar{S}\left(v^{n+1}\right) \rightarrow \bar{s}(w)
$$

We need the following

Lemma 3.8. Under the conditions of the theorem 3.3, If $w$ increases(decreases) in $\left(x_{j-\frac{1}{2}}, x_{j+\frac{3}{2}}\right)$, then

$$
v_{j+1}^{n+1} \geq(\leq) v_{j}^{n+1} .
$$

Proof. Assume that $w(x)$ monotonically increases. By (3.21),

$$
v_{j+1}^{n+1}-v_{j}^{n+1}=\frac{1}{h} \int_{-h / 2}^{h / 2}\left(\hat{w}\left(x_{j+1}+\xi\right)-\hat{w}\left(x_{j}+\xi\right)\right) d \xi .
$$

It suffices to show that

$$
\hat{w}\left(x_{j+1}+\xi\right) \geq \hat{w}\left(x_{j}+\xi\right), \quad \text { for }-h / 2<\xi<h / 2 .
$$


Notice that both $x_{j}+\xi$ and $x_{j+i}+\xi$ are in $\left(x_{j-\frac{1}{2}}, x_{j+\frac{3}{2}}\right)$. If $\left(x_{j}+\xi, x_{j+1}+\xi\right)$ contains no $\mathrm{d}$-point, inequality (3.30) is trivially true. If $\left(x_{j}+\xi, x_{j+1}+\xi\right)$ contains more than one $\mathrm{d}-$ point, say, $X_{k+\frac{1}{2}}, X_{k+\frac{3}{2}}, \ldots, X_{k+m+\frac{1}{2}}$, then by algorithm 3.1. and the monotonicity of $w(x)$, $\hat{w}\left(x_{j}+\xi\right) \leq v_{k+1}^{n} \leq \hat{w}\left(x_{j+1}+\xi\right)$. Finally, suppose that $\left(x_{j}+\xi, x_{j+1}+\xi\right)$ contains only one $\mathrm{d}$-point, say, $X_{k+\frac{1}{2}}$. Under the conditions of theorem 3.3., the inequality

$$
0<X_{j+\frac{1}{2}}-X_{j-\frac{1}{2}}<2 h
$$

holds for all $j$. Therefore, if we extend $\hat{w}(x)$ in $\left(X_{k-\frac{1}{2}}, X_{k+\frac{1}{2}}\right)$ linearly to $-\infty$, extend $\hat{w}(x)$ in $\left(X_{k+\frac{1}{2}}, X_{k+\frac{3}{2}}\right)$ linearly to $+\infty$, and denote the extension by $\hat{W}(x)$, then,

$$
\hat{W}\left(X_{k+\frac{1}{2}}+0\right) \geq \hat{W}\left(X_{k+\frac{1}{2}}-h\right)
$$

and

$$
\hat{W}\left(X_{k+\frac{1}{2}}+h\right) \geq \hat{W}\left(X_{k+\frac{1}{2}}-0\right) .
$$

The linearity of $\hat{W}(x)$ in each half-line devided by $X_{k+\frac{1}{2}}$ implies that for the above $\xi$, there is a $\theta$, satisfying $0<\theta<1$, such that

$$
\hat{w}\left(x_{j}+\xi\right)=\theta \hat{W}\left(X_{k+\frac{1}{2}}-h\right)+(1-\theta) \hat{W}\left(X_{k+\frac{1}{2}}-0\right) .
$$

and

$$
\hat{w}\left(x_{j+1}+\xi\right)=\theta \hat{W}\left(X_{k+\frac{1}{2}}+0\right)+(1-\theta) \hat{W}\left(X_{k+\frac{1}{2}}+h\right) .
$$

Clearly, (3.31)-(3.34) imply (3.30). The lemma is then proven.

Proof of theorem 3.3. We define a mapping $\mathbf{P}$ from $\bar{S}\left(v^{n+1}\right)$ into $\bar{s}(w)$ as follows.

Given $\bar{S}\left(v^{n+1} ; i, j\right) \in \bar{S}\left(v^{n+1}\right)$, one of the following five cases must occur. In each case, we will determine the image $\mathbf{P}\left(\bar{S}\left(v^{n+1} ; i, j\right)\right) \in \bar{s}(w)$, and prove that the mapping is well-defined.

Case 1. There is at least one strict maximum section $\bar{s}(w ; a, b) \in \bar{s}(w)$ such that

$$
[a, b] \cap\left[x_{i-\frac{1}{2}}, x_{j+\frac{1}{2}}\right] \neq \phi
$$

and

$$
\lim _{x \rightarrow a} \sup w(x) \geq v_{i}^{n+1}
$$


In this case, we pick up this strict maximum section to be $\mathbf{P}\left(\bar{S}\left(v^{n+1} ; i, j\right)\right)$. Obviously,

$$
x_{i-\frac{3}{2}}<a \leq b<x_{j+\frac{3}{2}}
$$

holds.

Case 2. The following two conditions hold:

1). There is no strict maximum section $\bar{s}(w ; a, b) \in \bar{s}(w)$ which satisfies the conditions of the case 1 ).

2). $w(x) \equiv v_{i}^{n+1}$, for $x_{i-\frac{1}{2}} \leq x \leq x_{j+\frac{1}{2}}$.

Let $(c, d)$ be the largest interval containing $\left(x_{i-\frac{1}{2}}, x_{j+\frac{1}{2}}\right)$ such that $w(x)$ is constant in it. Since $(c, d)$ is not a strict maximum section of $w(x), w(x)$ must either strictly increase in some right neighborhood of $x_{j+\frac{1}{2}}$ or strictly decrease in some left neighborhood of $x_{i-\frac{1}{2}}$. If the former is the case, according to the definition of $\bar{S}\left(v^{n+1} ; i, j\right)$, there must be a left most $\bar{s}(w ; a, b)$ such that

$$
d<a<b<x_{j+\frac{3}{2}}
$$

We then define $\mathrm{P}\left(\bar{S}\left(v^{n+1} ; i, j\right)\right)$ to be this $\bar{s}(w ; a, b)$. Otherwise we can pick up the right most one as $\mathrm{P}\left(\bar{S}\left(v^{n+1} ; i, j\right)\right)$. In either case, (3.35) holds.

Case 3. $w(x)$ increases but is not constant in $\left(x_{i-\frac{1}{2}}, x_{j+\frac{1}{2}}\right)$. We take $\mathbf{P}\left(\bar{S}\left(v^{n+1} ; i, j\right)\right)$ to be the left most $\bar{s}(w ; a, b) \in \bar{s}(w)$ such that $a \geq x_{j+\frac{1}{2}}$. The well-definedness is justified by above lemma, and (3.35) holds.

Case 4. $w(x)$ decreases but is not constant in $\left(x_{i-\frac{1}{2}}, x_{j+\frac{1}{2}}\right)$. We take $\mathbf{P}\left(\bar{S}\left(v^{n+1} ; i, j\right)\right)$ to be the right most $\bar{s}(w ; a, b) \in \bar{s}(w)$ such that $b \leq x_{i-\frac{1}{2}}$. The well-definedness is justified by the same lemma, and (3.35) holds.

Case 5. The following two conditions hold:

1). There is no strict maximum section $\bar{s}(w ; a, b) \in \bar{s}(w)$ which satisfies the conditions of the case 1.

2). $w(s)$ is not monotone in $\left(x_{i-\frac{1}{2}}, x_{j+\frac{1}{2}}\right)$.

In this case, there must be a $\xi$ in $\left(x_{i-\frac{1}{2}}, x_{j+\frac{1}{2}}\right)$ which satisfies the following requirements: a). $\hat{w} \equiv w$ in a neighborhood of $\xi$. b). $\hat{w}(\xi)=w(\xi)<v_{i}^{n+1}$ and c). Either

$$
\hat{w}\left[x_{i-\frac{1}{2}}, \xi\right]<v_{i}^{n+1}
$$


or

$$
\hat{w}\left[x_{i-\frac{1}{2}}, \xi\right]>v_{i}^{n+1}
$$

is true. If $(3.36)$ is the case, we define $\mathbf{P}\left(\bar{S}\left(v^{n+1} ; i, j\right)\right)$ to be the left most $\bar{s}(w ; a, b) \in \bar{s}(w)$ such that $a \geq x_{j+\frac{1}{2}}$. Otherwise, we define $\mathrm{P}\left(\bar{S}\left(v^{n+1} ; i, j\right)\right)$ to be the right most $\bar{s}(w ; a, b) \in$ $\bar{s}(w)$ such that $b \leq x_{i-\frac{1}{2}}$. To see that such $\bar{s}(w ; a, b)$ exists, let us assume that $(3.36)$ holds, then we have

$$
\hat{w}\left[\xi, x_{j+\frac{1}{2}}\right]>v_{j}^{n+1} .
$$

Suppose that $k$ is the integer satisfying

$$
X_{k+\frac{1}{2}} \geq x_{j+\frac{1}{2}}>X_{k-\frac{1}{2}}
$$

If $S_{k}^{n}<0$, then

$$
M=\sup _{x \in\left(\xi, x_{j+\frac{1}{2}}\right)} w(x)=\sup _{x \in\left(\xi, x_{j+\frac{1}{2}}\right)} \hat{w}(x)>v_{i}^{n+1} .
$$

Assume that $w(x)$ has been made continuous at those $\mathrm{d}$-points mentioned in the remark 3.6.. Then, $M$ will be attained by $w(x)$ in $\left(\xi, x_{j+\frac{1}{2}}\right)$. There is thus a strict maximum section $\bar{s}(w ; a, b)$ such that $\xi<a \leq b<x_{j+\frac{1}{2}}$ and

$$
\lim _{x \rightarrow a} \sup w(x) \geq v_{i}^{n+1}
$$

This contradicts the condition 1). Therefore one must have $S_{k}^{n} \geq 0$.

Using a similar argument one can show that $v_{k}^{n} \geq v_{j}^{n+1}$. Hence, if $\mathrm{w}(\mathrm{x})$ monotonically increases in $\left(x_{j+\frac{1}{2}}, x_{j+\frac{3}{2}}\right)$, then

$$
v_{j+1}^{n+1} \geq v_{k}^{n} \geq v_{j}^{n+1}
$$

which contradicts the definition of $\bar{S}\left(v^{n+1} ; i, j\right)$. This implies that there must be a $\bar{s}(w ; a, b)$ such that

$$
x_{j+\frac{3}{2}}>b \geq a>x_{j+\frac{1}{2}} .
$$

Similarly, if (3.37) holds, then, there must be a $\bar{s}(w ; a, b)$ such that

$$
x_{i-\frac{3}{2}}<a \leq b<x_{i-\frac{1}{2}}
$$


Finally, we have to show that $\mathrm{P}$ is $1-1$. Let $\bar{S}\left(v^{n+1} ; i_{1}, j_{1}\right)$, and $\bar{S}\left(v^{n+1} ; i_{2}, j_{2}\right)$ be two adjacent strict maximum sections of $v^{n+1}$, and

$$
\mathbf{P}\left(\bar{S}\left(v^{n+1} ; i_{k}, j_{k}\right)\right)=\bar{s}\left(w ; a_{k}, b_{k}\right), k=1,2
$$

From the argument above, we see that if

$$
\mathbf{P}\left(\bar{S}\left(v^{n+1} ; i, j\right)\right)=\bar{s}(w ; a, b),
$$

then

$$
x_{j-\frac{3}{2}}<a<b<x_{j+\frac{3}{2}} .
$$

Therefore, if $a_{1}=a_{2}$ and $b_{1}=b_{2}$, the only possibility would be

$$
i_{2}=j_{1}+2
$$

and

$$
x_{j_{1}+\frac{1}{2}}<a_{1} \leq b_{1}<x_{i_{2}-\frac{1}{2}}
$$

Now, one can see easily that either

$$
v_{j_{1}+1}^{n+1} \geq v_{j_{1}}^{n+1},
$$

which contradicts the definition of $\bar{S}\left(v^{n+1} ; i_{1}, j_{1}\right)$, or

$$
v_{i_{2}-1}^{n+1} \geq v_{i_{2}}^{n+1}
$$

which contradicts the definition of $\bar{S}\left(v^{n+1} ; i_{2}, j_{2}\right)$. The claim and, therefore, the theorem is proven.

Next, we consider the order of accuracy of our method. For linear equation

$$
u_{t}+u_{x}=0
$$

we have

Theorem 3.9. Suppose the $\alpha_{j}$ 's in algorithm 3.1. are uniformly bounded, then at the worst, this method lowers the accuracy of the original scheme by one order. If, in addition,

$$
\alpha_{j+1}-\alpha_{j}=O(h)
$$


then the method applied to the ENO schemes keeps the order of the accuracy. For the choices of the parameters we are going to suggest, this last condition is valid if there are no zeros of the first $r-1$ derivatives of the true solution $u$ near $x_{j}$

Proof. It suffices to recall that, at the cell interface $x_{j+\frac{1}{2}}$, the jump $\delta_{j+\frac{1}{2}}$ of the reconstruction satisfies

$$
\cdot \delta_{j+\frac{1}{2}}=c_{j+\frac{1}{2}} h^{r}+O\left(h^{r+1}\right)
$$

and

$$
\Delta c_{j+\frac{1}{2}}=O(h)
$$

if

$$
\left(\frac{d}{d x}\right)^{l} u(x) \neq 0, \quad l=1,2, \ldots, r-1 .
$$

We believe that the conclusion of above theorem is also true for the fully nonlinear problems, but do not have a proof yet.

Next, we discuss the choice of the $\alpha_{j}$ 's. Hereafter we shall call them the SM(slope modification) coefficients. Unfortunately, we have not been able to give a universal method for determining the $\alpha_{j}$ 's. The recommendations we are going to give come from our numerical experiments. We found that, for linear problems, the smearing effect is essentially eliminated when the $\alpha_{j}$ 's are greater or equal to $1.9-2.3$. One usually gets satisfactory results by letting $\alpha_{j}$ be equal to or slightly larger than the smallest number capable of eliminating the smearing effect.

For the problems with rich smooth structures, the uniform choice of $\alpha_{j}$ as suggested above could run into trouble. Although the essentially non-oscillatory property is generally kept, some artificial contact discontinuities may damage the smooth structure.

One way of avoiding this is to use a discontinuity detector. A well known one is

$$
\mu_{j}=\left(\frac{\Delta^{2} u_{j-1}}{\left|\Delta u_{j-1}\right|+\left|\Delta u_{j}\right|}\right)^{2}
$$

Then $\alpha_{j}$ can be written as

$$
\alpha_{j}=c \mu_{j}
$$

In most computations we have performed, $c=33$. 
Remark 3.10. Usually,(3.43) and (3.44) give satisfactory results. However, for the local CFL number far away from \pm 0.5 , the compression effect is not balanced between the head and the tail of a discontinuity. Further improvement can be made by multiplying the coeffcients (3.44) with a balance factor. For details of the balance factor, see $\S 5$.

\section{\$4 Application to the ENO schemes for the Euler equations of gas dynamics}

In this section, we apply the slope modifying method to the ENO schemes for the Euler equations of gas dynamics for a polytropic gas. The application to the general systems of hyperbolic conservation laws follows immediately.

For a polytropic gas, the governing equations are, as in [4],

$$
\begin{gathered}
u_{t}+f(u)_{x}=0 \\
u=(\rho, m, E)^{T} \\
f(u)=q u+(0, P, q P)^{T} \\
P=(\gamma-1)\left(E-\frac{1}{2} \rho q^{2}\right)
\end{gathered}
$$

Here $\rho, q, P$, and $E$ are the density, velocity, pressure and total energy, respectively; $m=\rho q$ is the momentum and $\gamma$ is the ratio of specific heats.

The eigenvalues of the Jacobian matrix $\frac{\partial f}{\partial u}$ are

$$
a_{1}(u)=q-c, a_{2}(u)=q, a_{3}(u)=q+c
$$

where $c=(\gamma P / \rho)^{\frac{1}{2}}$ is the sound speed.

The corresponding right-eigenvectors are

$$
r_{1}(u)=\left(\begin{array}{c}
1 \\
q-c \\
H-q c
\end{array}\right), r_{2}(u)=\left(\begin{array}{c}
1 \\
q \\
\frac{1}{2} q^{2}
\end{array}\right), r_{3}(u)=\left(\begin{array}{c}
1 \\
q+c \\
H+q c
\end{array}\right)
$$

here

$$
H=(E+P) / \rho=c^{2} /(\gamma-1)+\frac{1}{2} q^{2}
$$

is the enthalpy. 
The corresponding left-eigenvectors bi-orthonormal to (4.6) are

$$
\left\{\begin{array}{l}
l_{1}(u)=\frac{1}{2}\left(b_{2}+q / c,-b_{1} q-1 / c, b_{1}\right) \\
l_{2}(u)=\left(1-b_{2}, b_{1} q,-b_{1}\right) \\
l_{3}(u)=\frac{1}{2}\left(b_{2}-q / c,-b_{1} q+1 / c, b_{1}\right)
\end{array}\right.
$$

where

$$
\begin{gathered}
b_{1}=(\gamma-1) / c^{2} \\
b_{2}=\frac{1}{2} q^{2} b_{1}
\end{gathered}
$$

To avoid too many collisions of the discontinuities which damage the advantage of the ENO reconstructions, ENO uses the characteristic reconstruction. Numerical experiments demonstrate that our slope modifier should also be applied in characteristic variables to get rid of some, although minor, spurious oscillations. In addition, since our goal is to sharpen the contact discontinuities which are the 2-waves, we only have to use it in the second field. We now introduce two algorithms to modify the $r$-th order characteristic reconstruction.

\section{Algorithm 4.1. For each $j$,}

1). Compute the locally defined characteristic variables

$$
\begin{aligned}
\bar{w}_{i}^{k}\left(v_{j}^{n}\right)=l_{k}\left(v_{j}^{n}\right) v_{i}^{n} \quad \text { for } i=j-r, \ldots, j+r \quad \text { if } k=1, \hat{3}, \\
\text { for } i=j-r-1, \ldots, j+r+1 \quad \text { if } k=2 .
\end{aligned}
$$

2). Apply the scalar reconstruction algorithm to each of the locally defined characteristic variable in (4.11). The result is

$$
b_{j, l}^{k}=\left.\frac{\partial^{l}}{\partial x^{l}} R\left(x ; \bar{w}^{k}\left(v_{j}^{n}\right)\right)\right|_{x=x}, \quad l=0,1, \ldots, r-1 ; k=1,3,
$$

and

$$
\left.\frac{\partial^{l}}{\partial x^{l}} R\left(x ; \bar{w}^{2}\left(v_{j}^{n}\right)\right)\right|_{x=x_{j+m}}, \quad l=0,1, \ldots, r-1 ; m=-1,0,1 .
$$

When $m=0, l \neq 1$, we denote (4.13) by $b_{j, l}^{2}$.

3). Apply our method, i.e., add the slope modifier to (13) to get $b_{j, 1}^{2}$.

4). Transform back to physical variables:

$$
R\left(x ; v^{n}\right)=\sum_{l=0}^{r-1} \vec{b}_{j, l}\left(x-x_{j}\right)^{l} / l ! \quad x_{j-\frac{1}{2}}<x<x_{j+\frac{1}{2}}
$$


where

$$
\vec{b}_{j, l}=\sum_{k=1}^{3} b_{j, l}^{k} r_{k}
$$

Algorithm 4.2. 1). For each $j$, do the ENO characteristic reconstruction as usual to get $R\left(x, v^{n}\right)$ for $x_{j-\frac{1}{2}}<x<x_{j+\frac{1}{2}}$. Store $l_{2}\left(v_{j}^{n}\right), \quad r_{2}\left(v_{j}^{n}\right), \quad \bar{w}_{j-1}^{2}\left(v_{j}^{n}\right), \quad \bar{w}_{j}^{2}\left(v_{j}^{n}\right), \quad \bar{w}_{j+1}^{2}\left(v_{j}^{n}\right)$, and $b_{j, l}^{2} \cdot$

2). For each $j$, compute $R_{j+\frac{1}{2}}^{ \pm}=R\left(x_{j+\frac{1}{2}} \pm 0, v^{n}\right), \delta_{j+\frac{1}{2}}=R_{j+\frac{1}{2}}^{+}-R_{j+\frac{1}{2}}^{-}$.

3). For each $j$,

i). compute

$$
\begin{aligned}
& R^{+}(w)=l_{2}\left(v_{j}\right) R_{j+\frac{1}{2}}^{-}, \\
& R^{-}(w)=l_{2}\left(v_{j}\right) R_{j-\frac{1}{2}}^{+}, \\
& \delta^{+}(w)=l_{2}\left(v_{j}\right) \delta_{j+\frac{1}{2}}
\end{aligned}
$$

and

$$
\delta^{-}(w)=l_{2}\left(v_{j}\right) \delta_{j-\frac{1}{2}}
$$

ii). using $\bar{w}_{j-1}^{2}\left(v_{j}^{n}\right), \quad \bar{w}_{j}^{2}\left(v_{j}^{n}\right), \quad \bar{w}_{j+1}^{2}\left(v_{j}^{n}\right), \quad R^{+}(w), \quad R^{-}(w), \quad \delta^{+}(w)$, and $\delta^{-}(w)$ to get the slope modifier $\partial b_{j, 1}^{2}$,

iii). compute $\vec{b}_{j, 1}=\vec{b}_{j, 1}+\partial b_{j, 1}^{2} r_{2}\left(v_{j}^{n}\right)$.

\section{$\S 5$ Numerical results}

Since our purpose in designing the slope modification method is to improve the performance of the ENO schemes for the solutions which contain contact discontinuities, the numerical experiments we will present in this section are either on linear equations with discontinuous initial values or on the Euler equations for gas dynamics whose solutions contain contact discontinuities. All the examples are standard problems widely used in the literature to test various schemes for the hyperbolic conservation laws.

With these examples, in addition to showing the performance of our method, we also try to give some suggestions on how to choose the parameters in our algorithms since we have not been able to establish any theoretically meaningful rule to determine these parameters. 
Example 5.1. We apply our method to 1D linear model problem

$$
\begin{gathered}
u_{t}+u_{x}=0, \quad 1 \leq x \leq 1 \\
u(x, 0)=u_{0}(x)
\end{gathered}
$$

with periodic boundary condition.

We test our method for this problem with each of the following four initial conditions. Divide the interval $[-1,1]$ into 100 cells of equal size. The center of the $\mathrm{j}$-th cell is $x_{j}=$ $(j-50.5) h$, where $h=1 / 50$. The four initial conditions are

$$
\begin{gathered}
v_{j}^{0}= \begin{cases}1, & 35 \leq j \leq 65, \\
0, & \text { otherwise }\end{cases} \\
v_{j}^{0}= \begin{cases}\left(1-[(j-50) / 15]^{2}\right)^{1 / 2}, & 35 \leq j \leq 65, \\
0, & \text { otherwise }\end{cases} \\
v_{j}^{0}=e^{-300\left(x_{j}-0.5\right)^{2}}
\end{gathered}
$$

and

$$
v_{j+25}^{0}(\bmod 100)= \begin{cases}-y_{j} \sin \left(\frac{3}{2} \pi y_{j}^{2}\right), & -1 \leq-\frac{1}{3}, \\ \left|\sin \left(2 \pi y_{j}\right)\right|, & \left|y_{j}\right|<\frac{1}{3}, \\ 2 y_{j}-1-\sin \left(3 \pi y_{j}\right) / 6, & \frac{1}{3}<y_{j}<1 .\end{cases}
$$

In the last condition, $y_{j}=x_{j}-0.01$. Essentially, the first 3 conditions are those used by Zalesak [15] (see also the references therein) and the last one is that used by Harten et al [4] and [9]

The numerical solutions for the initial condition (5.3) are displayed in the Figures 1 19. In the computations for the Figures 1-5, the 2nd order ENO scheme is used. The CFL number is fixed at 0.8 and the SM coefficients $\alpha_{j}$ 's in (3.4) are chosen to be independent of $j$ and $n$. We increase $\alpha_{j} \equiv \alpha$ from 0 to 10 and, for each choice of $\alpha$, run the program twice for 250 timesteps and 1250 timesteps respectively. The figures show clearly that, for small $\alpha$, the discontinuities are smeared more and more as the the number of the time steps 
increases. However when the coefficient reach about 1.9-2.3, the smearing appears to be essentially eliminated. This is indicated by the coincidence of the graphs of the numerical solutions obtained by running the program over the two different numbers of time steps. For a given ENO scheme and a fixed CFL number, we call the smallest $\alpha$ which is capable of eliminating the smearing effect the critical value of the SM coefficient with respect to the scheme and the CFL number.

Remark 5.1. A quantity equal to or slightly larger than the superemum of the critical value over the range $[0,1]$ of the CFL number is a good candidate for the choice of the MS coefficient. For the solutions which lack smooth structure we can choose a larger SM coefficient. Otherwise, we should choose one near or equal to the superemum of the critical value.

Remark 5.2. For the solution dependent SM coefficient (3.44), one can similarly "define" the critical value for the parameter $c$. The recommondations in the last remark also applies here.

The Figures 6-13 show the effect of CFL numbers on the critical values. We do the same computations as for the Figure 1-5 with different CFL numbers. The SM coefficients are $\alpha=1.9$ and $\alpha=2.3$. We found that the effect is minor.

Notice that although the CFL numbers have little effect on the critical value of the SM coefficients, it does play a role in the profiles of the solutions over the linear discontinuities. The sharpening effect is not well balanced, i.e., the steepness at the heads of the discontinuities is different from that at the tails when the CFL numbers are far away from \pm 0.5 . Looking at the Figure 1 carefully, one sees that this property of nonsymmetry already exists with the original ENO schemes.

For solutions with rich smooth structures and/or for multi-dimensional problems, when our slope modification method is applied and the resulted programs are run for huge number of timesteps, this phenomenon becomes more serious. To overcome this trouble, observe that at the head of a discontinuity, $\left|\frac{\Delta_{j-1} v}{\Delta_{j} v}\right|$ is very large, while at the tail, it is very small. We hence define the following balance operator

$$
\beta_{j}\left(\lambda_{j}, b\right)=\left|\frac{\triangle_{j-1} v}{\triangle_{j} v}\right|^{b\left(\lambda_{j}-0.5 \operatorname{sgn}\left(\lambda_{j}\right)\right)}
$$


where $b$ is a positive parameter to be chosen, $\lambda_{j}=\bar{a}_{j} \frac{\tau}{h}$ is the local CFL number, and $\bar{a}_{j}$ is the characteristic speed. We will see the effect of this operator in the examples displayed in the Figures $26-29$.

The Figures 12-19 show the effect of the order of accuracy on the the critical values of the SM coefficients. In these figures, $r-0$ denotes the " $r$-th order" ENO schemes, while $r-1$ denotes the $r$-th order ENO schemes, both in the sense of [4]. We find that the effect is also minor. This is interesting since the jumps of a higher order reconstruction at the cell interfaces over the transitions of the discontinuities are much smaller than those of a lower order reconstruction (see, again, the figures at the end of [4]).

The fact that the critical value of the SM coefficients is relatively independent of rather than inversely proportional to the jumps for different orders of the schemes is consistent to the fact that the higher order ENO schemes smear the contact discontinuities less then the lower order ones do.

The results for the initial condition (5.4) are displayed in the Figures $20-29$. In the Figures $20-25$, we use the SM coefficient 1.9. The improvement is apparent. The Figures $26-29$ test the effect of the balance operator (5.7). This time we run our program of the 2nd order ENO for 6000 timesteps. For the Figures 26 and 27, we use the constant SM coefficients 1.9 while for the Figures 28 and 29 , we use (3.44) with $c=33$. The results obtained by using the SM coefficients multiplied by the balance operator with $b=4.3$ are displayed in the Figures 26 and 28. The Figures 27 and 29 show the corresponding results without applying the operator.

The Figures 30-31 displays the numerical results for initial condition (5.5); the Figures 32-33 are the results for initial condition (5.6). Again one can see apparent improvements of our method in the treatment of the cusps, jumps or both.

Example 5.2 We apply our method to the Riemann problems for the Euler quations of gas dynamics (4.1) with following two sets of initial conditions known as the Sod problem and the Lax problem respectively:

$$
\begin{aligned}
& \left(\rho_{L}, q_{L}, P_{L}\right)=(1,0,1) \\
& \left(\rho_{R}, q_{R}, P_{R}\right)=(0.125,0,0.10)
\end{aligned}
$$


and

$$
\begin{aligned}
& \left(\rho_{L}, q_{L}, P_{L}\right)=(0.445,0.698,3.528) ; \\
& \left(\rho_{R}, q_{R}, P_{R}\right)=(0.5,0,0.571)
\end{aligned}
$$

Both problems are solved by the "4-th order" ENO schemes with and without the slope modification. In all the computations, we use 100 equally sized space cells and the CFL number 0.8. The Figures 34 and 35 are the numerical results for Sod problem after 60 time steps; the Figures 36 and 37 are those for Lax problem after 85 timesteps. Here we apply the alghorithm 4.1 with the SM coefficients (3.44) such that $c=33$. Notice that with the slope modification method the computed contact discontinuities are of the same quality as the discontinuities captured by the same method in the linear model problems above.

Example 5.3 (The blast waves problem). Here we consider the equations (4.1) with the following initial condition

$$
u(x, 0)= \begin{cases}u_{L}, & 0 \leq x<0.1 \\ u_{M}, & 0.1 \leq x<0.9 \\ u_{R}, & 0.9 \leq x<1\end{cases}
$$

where

$$
\begin{aligned}
& \rho_{L}=\rho_{M}=\rho_{R}=1, \quad q_{L}=q_{M}=q_{R}=0, \\
& P_{L}=10_{3}, P_{M}=10^{-2}, P_{R}=10^{2},
\end{aligned}
$$

and the two boundaries are assumed to be solid walls. See [14] for the details of the solution and the comparison of the performance of various schemes for this problem. Notice that the contact discontinuity results from the collision of the two strong shocks. It is extremely difficult to be "captured" by a shock capturing scheme in the Eulerian framework. In fact, no scheme tested in [14] captured the contact discontinuity well. To the auther's knowledge, in the literature of the modern shock capturing technique, the only successful computations of this discontinuity so far have been performed by Harten's subcell resolution(see [9] and [7]). In the computations for this problem, we use 200 cells and the CFL number 0.8 . The results at $t=0.038$ are displayed in the Figures 38,39 and 40. The solid lines are the numerical solutions obtained by the 2nd order ENO scheme and the slope modification method with 800 cells. The Figure 38 is the result of the 2 nd order ENO scheme with the algorithm 4.2 
and the Figure 39 is the result of the 4-th order ENO schemes with the algorithm 4.1 . For comparison, in the Figure 40 we display the numerical result of the 4-th order ENO without the slope modification. Notice that all the contact discontinuities are well captured by the ENO schemes with our method. Notice also that the two algorithm perform equally well.

Example 5.4 To see the performance of our method for the problems that have some structure, we apply it to the Euler equations (4.1) with the following initial condition:

$$
(\rho, q, P)= \begin{cases}(3.857143,2.629369,10.33333), & x<-4 \\ (1+0.1 \sin 5 x, 0,1), & x \geq-4\end{cases}
$$

This is a model problem for shock/turbulence interaction. See [12] for a linearized analysis of this problem, and [7] for a numerical result. We apply our slope modification method with the balance operator on the "4-th order" ENO schemes. The results are demonstrated in the Figures 41-34. The computations are performed with the CFL number 0.8. The solid line is the result with 800 cells. Comparing the result with that in [7] we can regard it as the exact solution. The circles in the Figures 41 and 42 are the results computed with 200 cells and 400 cells respectively. For comparison, the Figures 43 and 44 show the results computed with 200 cells and 400 cells respectively without the slope modification.

Example 5.5 This is a preliminary result for $2 \mathrm{D}$ computations. Consider the $2 \mathrm{D}$ model problem

$$
u_{t}+u_{x}+u_{y}=0,-1 \leq x, y \leq 1
$$

with the initial condition

$$
u_{0}(x, y)= \begin{cases}1, & |x-y| \leq \frac{1}{2} \\ 0, & \text { otherwise }\end{cases}
$$

In [10], Harten used this problem to test the performance of the ENO schemes with the 2D reconstructions via deconvolution. Now, we use the reconstructions via primitive functions dimension by dimension and, at the same time, apply the scalar slope modification algorithm with the balance operator. The CFL number is $0.8 \times 0.8$. The computations are performed with $20 \times 20$ cells. The results with the slope modification are displayed in the Figures $45-47$, while those without in the Figures 48-50. The apparent improvements shown in these re- 
sults and the results in [7] indicate that the present method is promising in $2 \mathrm{D}$ computations.

Acknowledgement. I would like to express my highest appreciation for Professor S. Osher's invaluable guidance, encouragement and support throughout the period of this work. I am also extremely grateful to Professor A. Harten for many helpful discussions and instructions. Thanks also to C.-W. Shu, W. E, D. Mao and R. Donat for helpful discussions and/or pointing out the errors in the manuscript. 


\section{References}

[1] A. Harten, "The artificial compression method for computation of shocks and contact-discontinuities: III. self-adjusting hybrid schemes,” Math. Comp., Vol. 32 (1978), pp. $363-389$.

[2] A. Harten, S. Osher, "Uniformly high order non-oscillatory schemes, I.," SINUM, Vol. 24 (1987), pp. 279 - 309.

[3] A. Harten, S. Osher, B. Engquist and S. Chakravarthy, "Some results on uniformly high order non-oscillatory schemes," J. Appl. Numer. Math., Vol. 2 (1986), pp. 347.

[4] A. Harten, B. Engquist, S. Osher and S. Chakravarthy, "Uniformly high order nonoscillatory schemes, III.," J. Comp. Phys., Vol. 71 (1987), pp. 231.

[5] S. Osher and P. K. Sweby, "Recent developments in the numerical solution of non-linear conservation laws," in "The State of the art in numerical analysis", pp. 681 - 701, eds. A. Iserles, M. Powell, Clarendon Press, Oxford, (1987).

[6] C.-W. Shu and S. Osher, "Efficient implementation of essentially non-oscillatory shock capturing schemes," ICASE Report 87-33, 1987, to appear in J. Comp. Phys..

[7] C.-W. Shu and S. Osher, "Efficient implementation of essentially non-oscillatory shock capturing schemes, II.," to appear in J. Comp. Phys..

[8] E. Katzer and S. Osher, "Efficient implementation of essentially non-oscillatory schemes for nonlinear hyperbolic differential eguations," CAM Report 88-14(May 1988)

[9] A. Harten, "ENO schemes with subcell resolutions," ICASE Report 87-56 (August 1987).

[10] A. Harten, "Preliminary results on the extension of the ENO schemes to two dimensional problems," in Proceedings of the international conference on hyperbolic problems (SaintEtienne, January 1986).

[11] D. K. Mao, "A treatment of discontinuities in shock capturing finite difference methods I. Single conservation law," preprint. 
[11] D: K. Mao, "A treatment of discontinuities in shock capturing finite difference methods I. Single conservation law," preprint.

[12] J. F. McKenzie and K. O. Westphal, "Interaction of linear waves with oblique shock waves," Phys. Fluids, Vol 11 (1968), pp. 2350-2362.

[13] P.D. Lax and B. Wendroff, "Systems of conservation laws," Comm. Pure Appl. Math., Vol. 13 (1960), pp. 217-237.

[14] P. Woodward and P. Colella, "The numerical simulation of two- dimensional fluid with strong shocks," J. Comp. Phys., Vol. 54 (1984) pp. 115 - 173.

[15] S. Zalesak, "A preliminary comparison of modern shock-capturing schemes: linear advection," In Advances in computer methods for partial differential equations, VI, edited by R. Vichnevetsky and R.Stepleman, IMACS, 1987. 

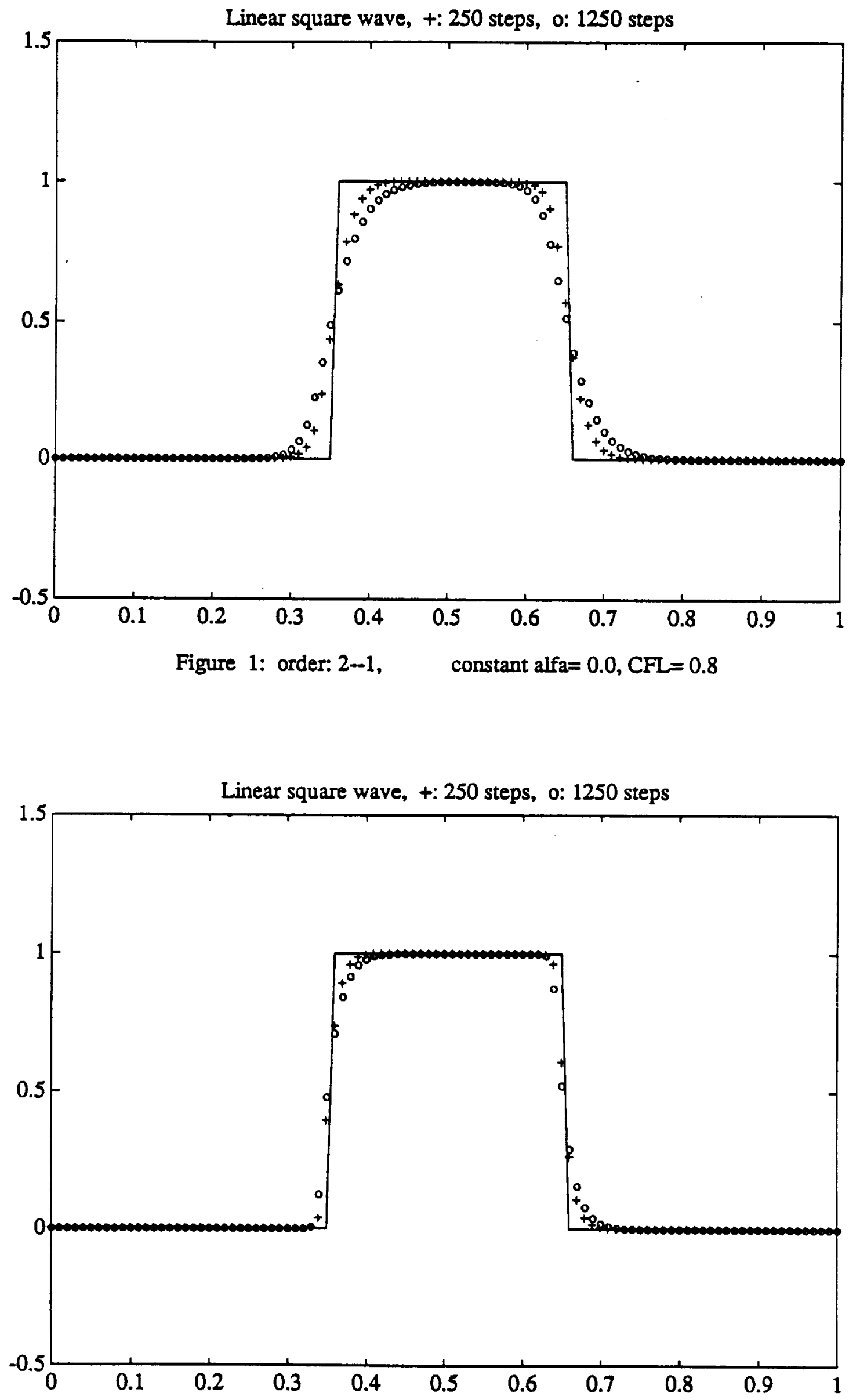

Figure 2: order: 2--1, $\quad$ constant alfa $=1.5, \mathrm{CFL}=0.8$ 


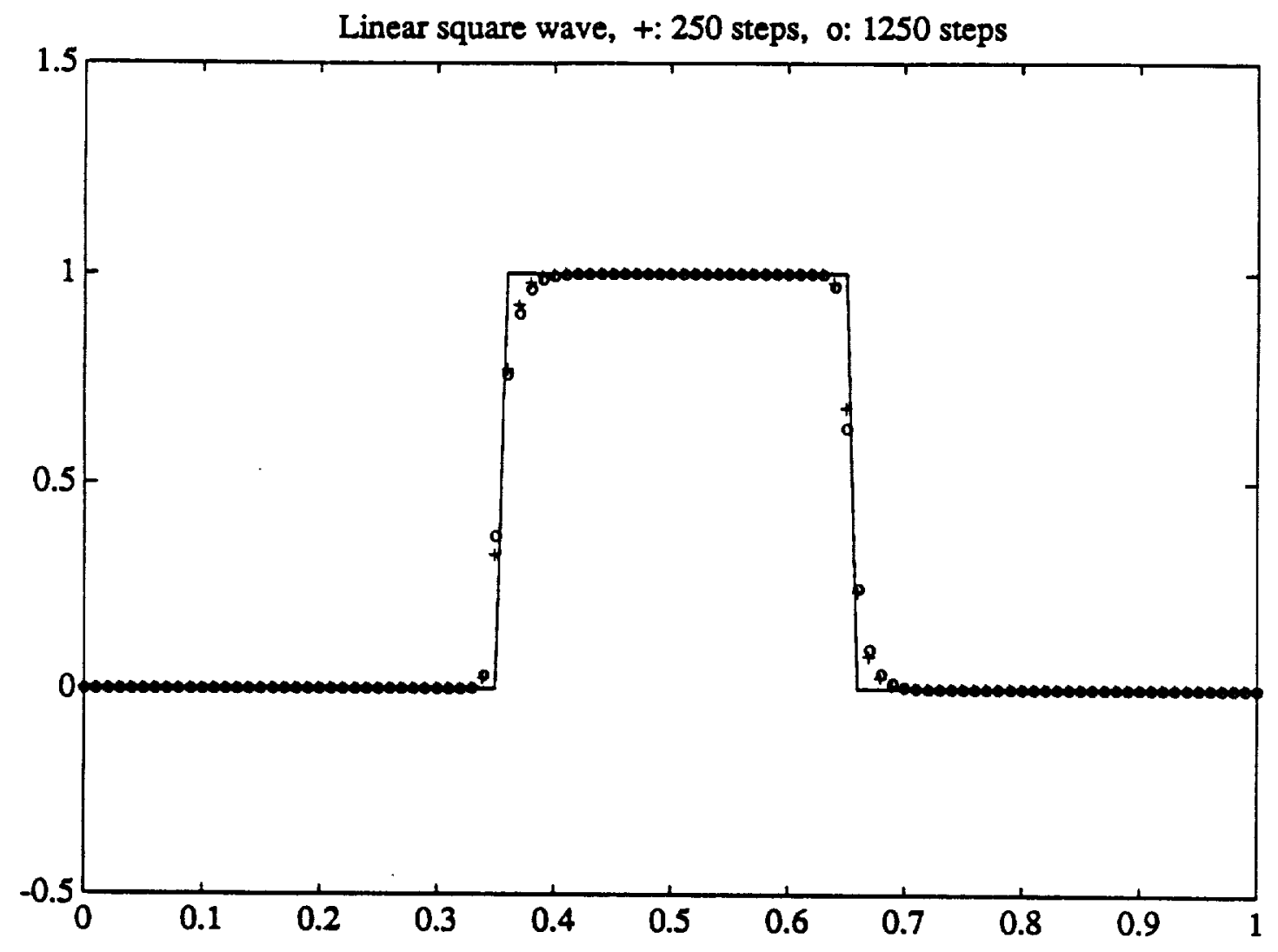

Figure 3: order: $2-1, \quad$ constant alfa $=1.9, C F L=0.8$

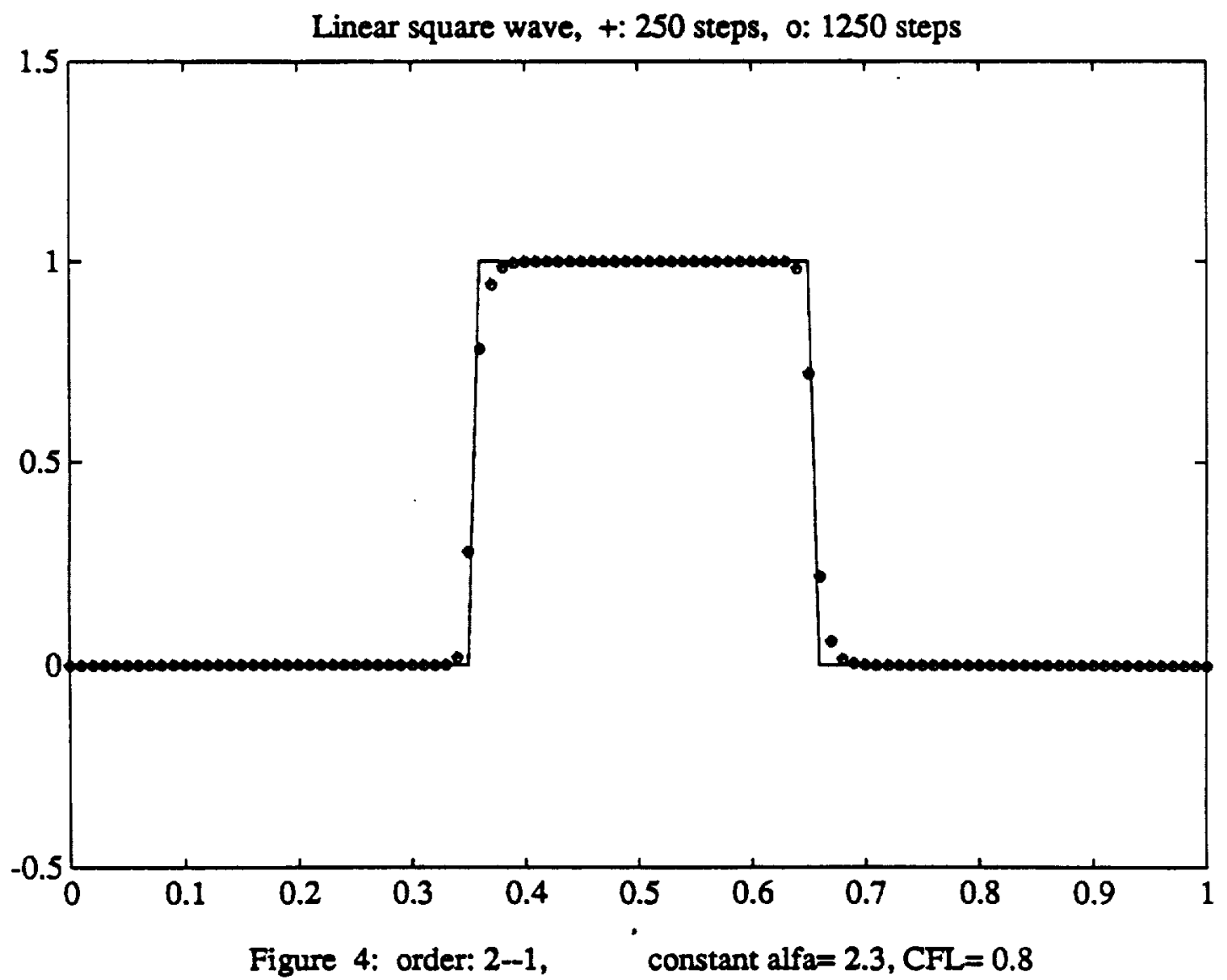




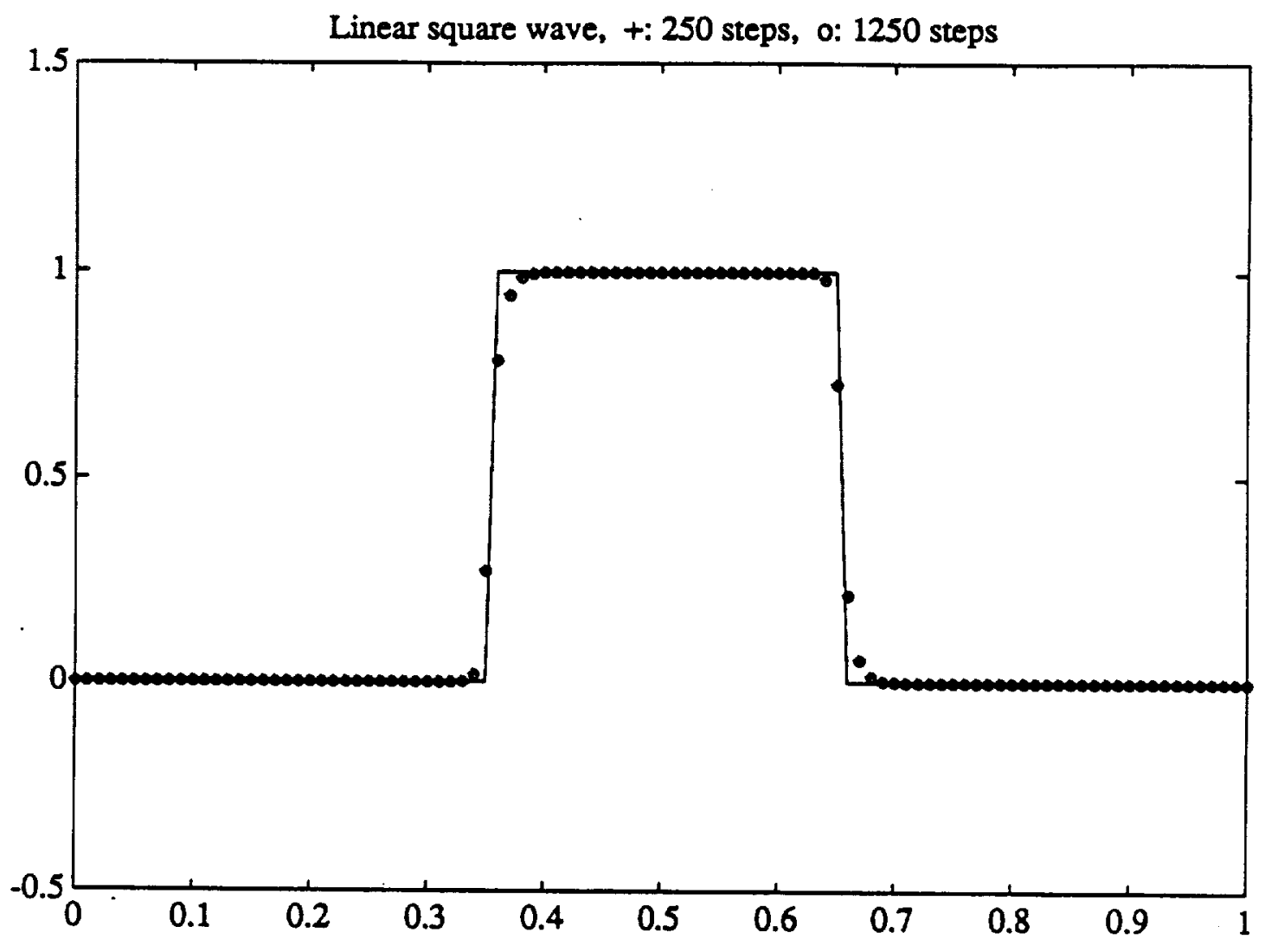

Figure 5: order: $2-1, \quad$ constant alfa $=10.0, C F L=0.8$

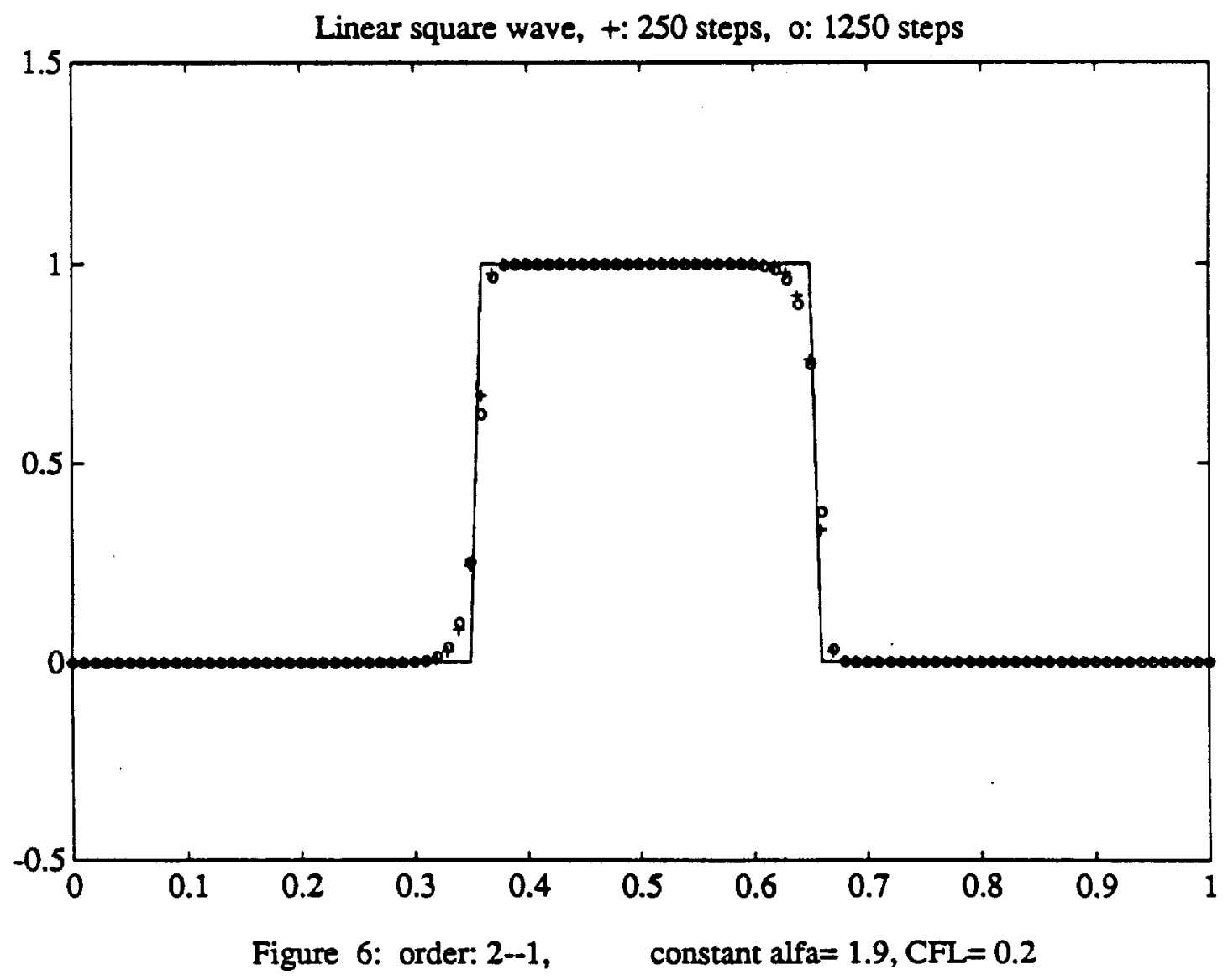




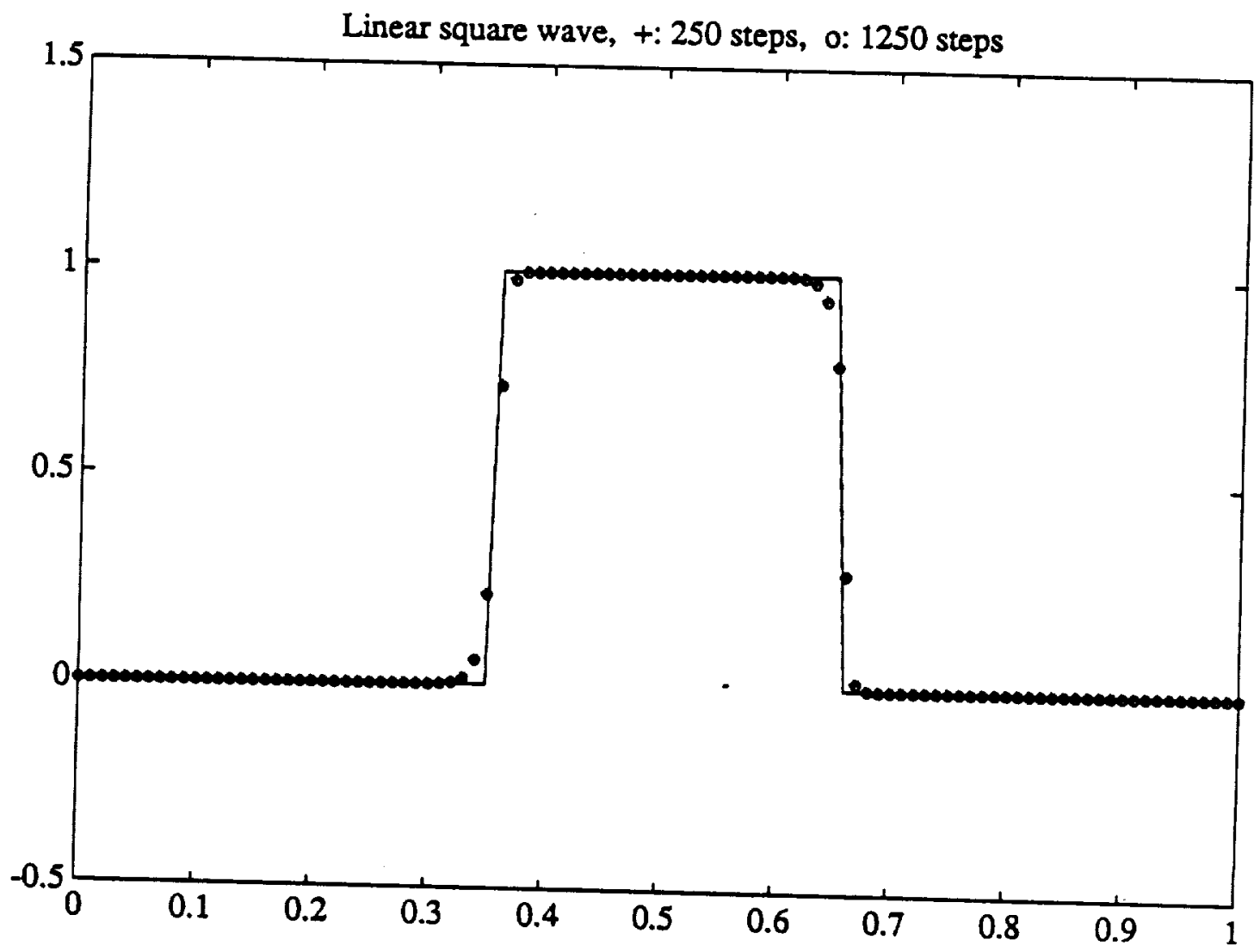

Figure 7: order: $2-1, \quad$ constant alfa $=2.3, C F L=0.2$

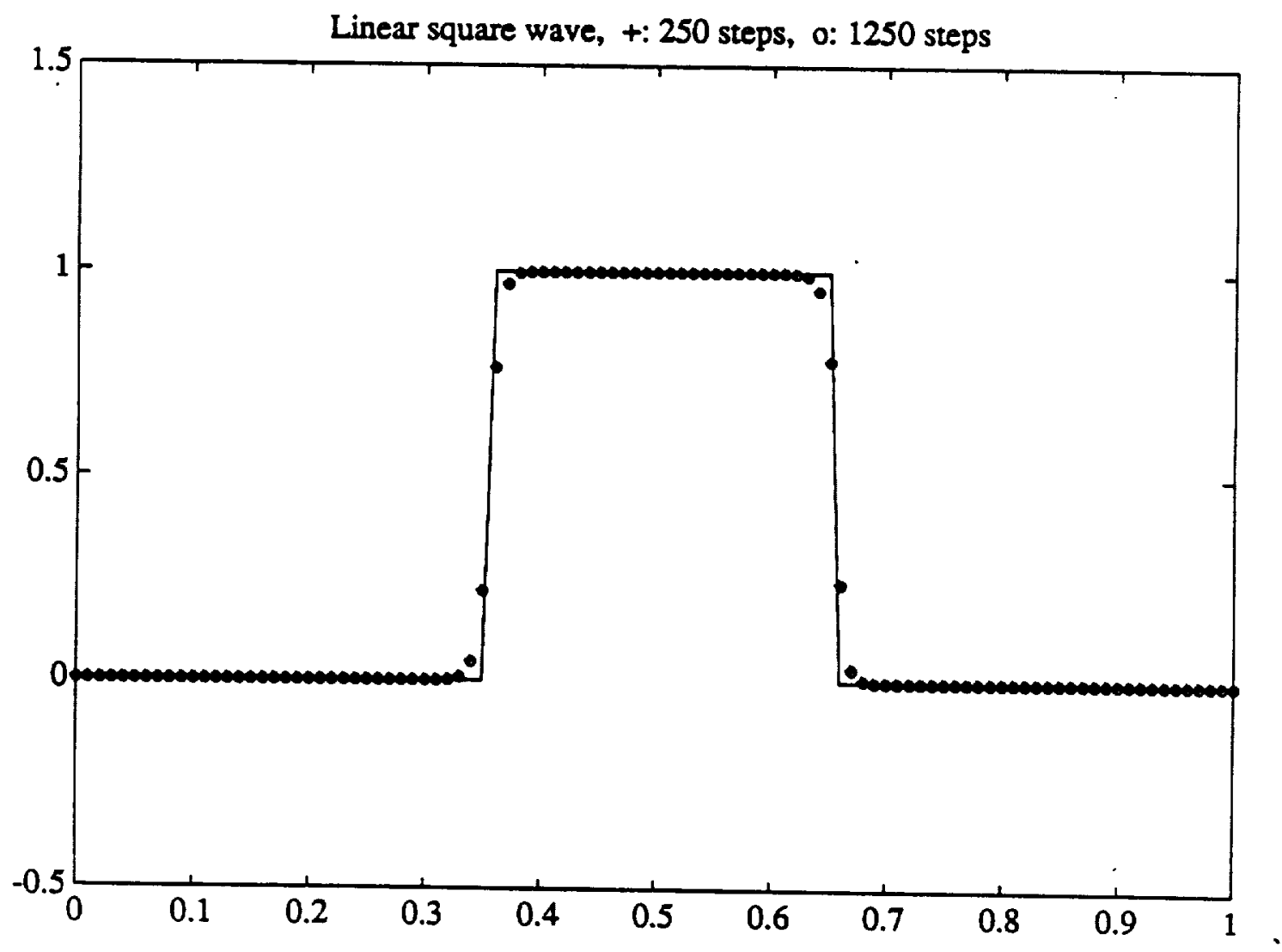

Figure 8: order: $2-1, \quad$ constant alfa $=1.9, C F L=0.4$ 


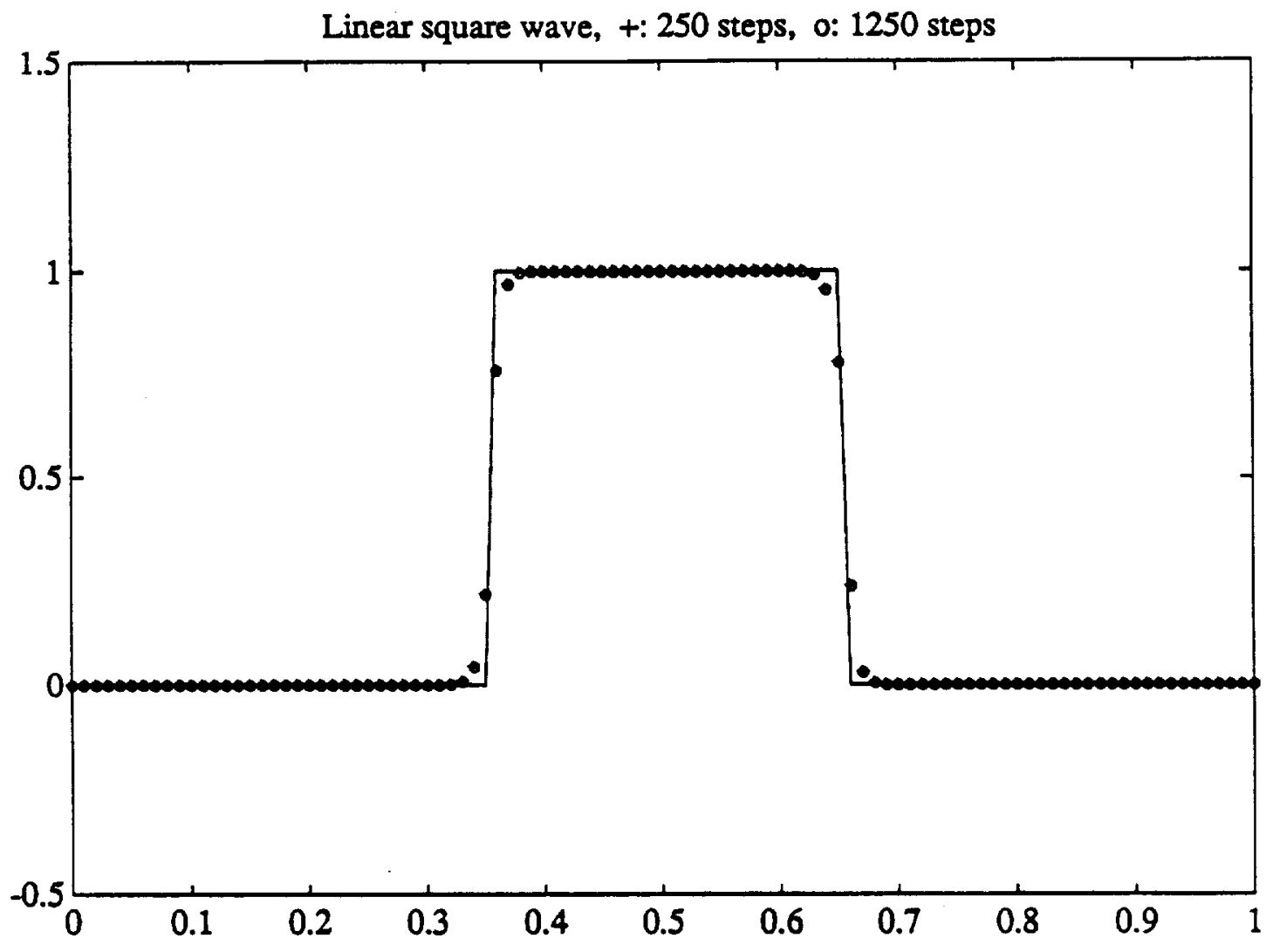

Figure 9: order: $2--1, \quad$ constant alfa $=2.3, \mathrm{CFL}=0.4$

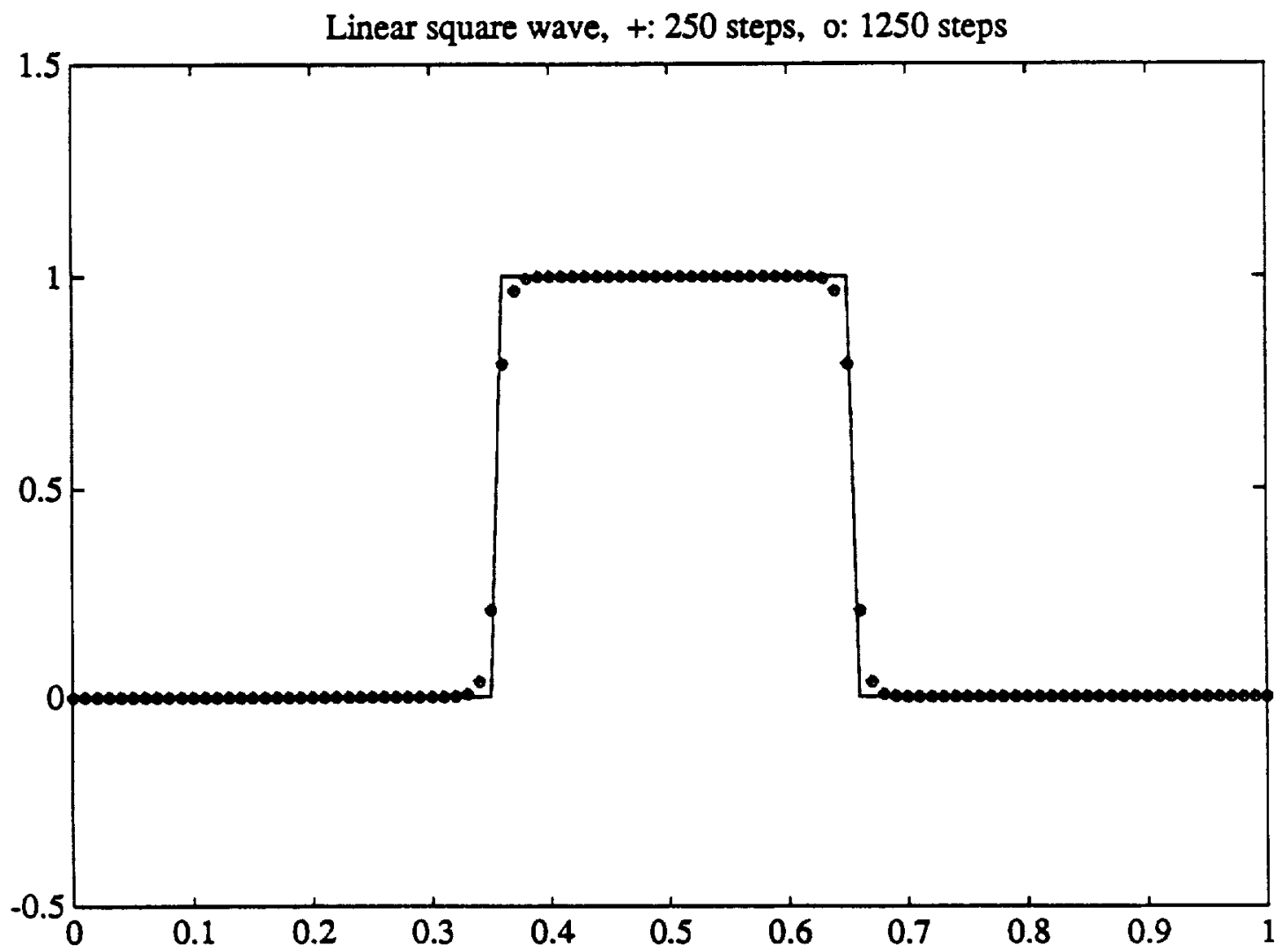

Figure 10: order: $2-1, \quad$ constant alfa $=1.9, \mathrm{CFL}=0.5$ 


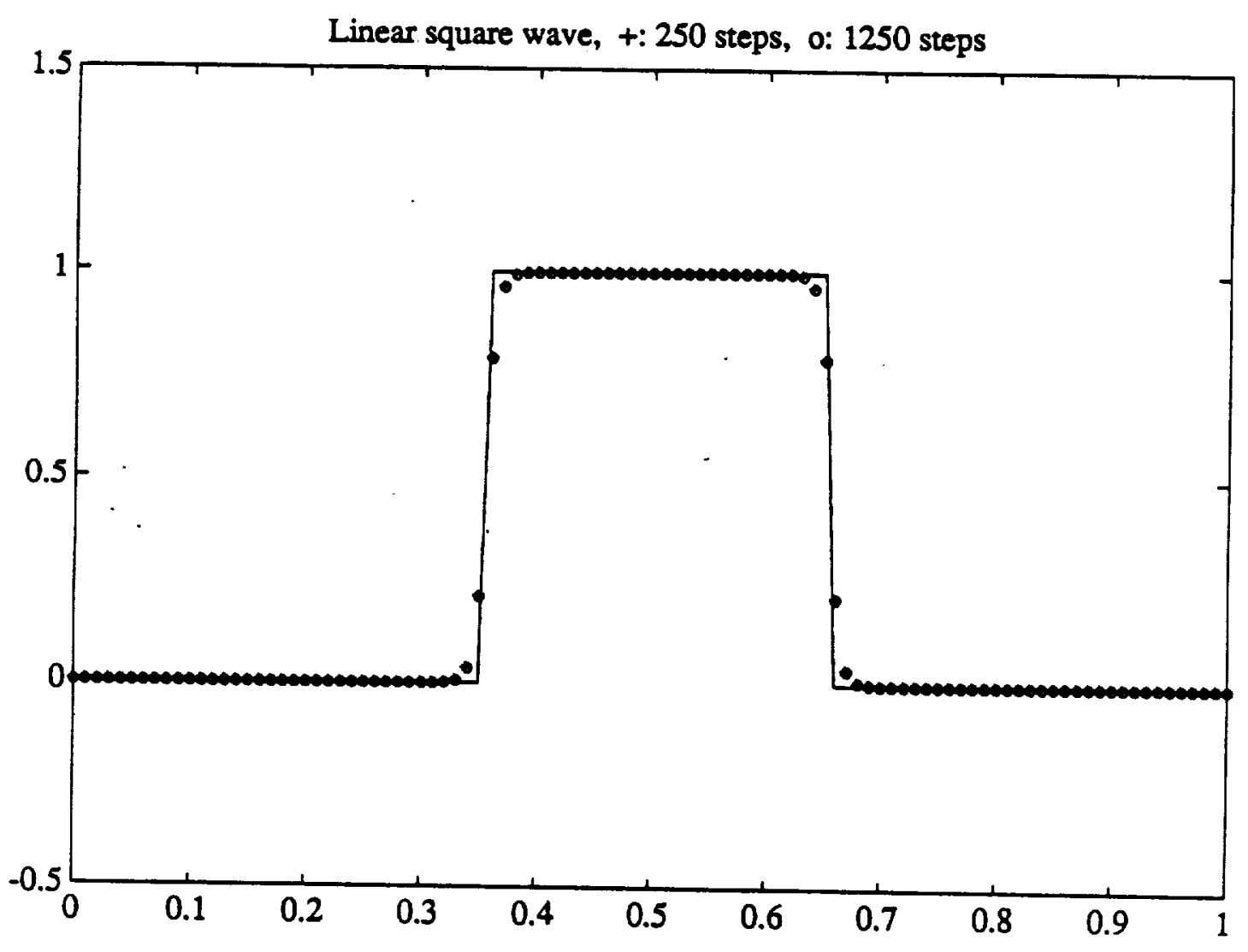

Figure 11: order: $2-1, \quad$ constant alfa $=2.3, C F L=0.5$

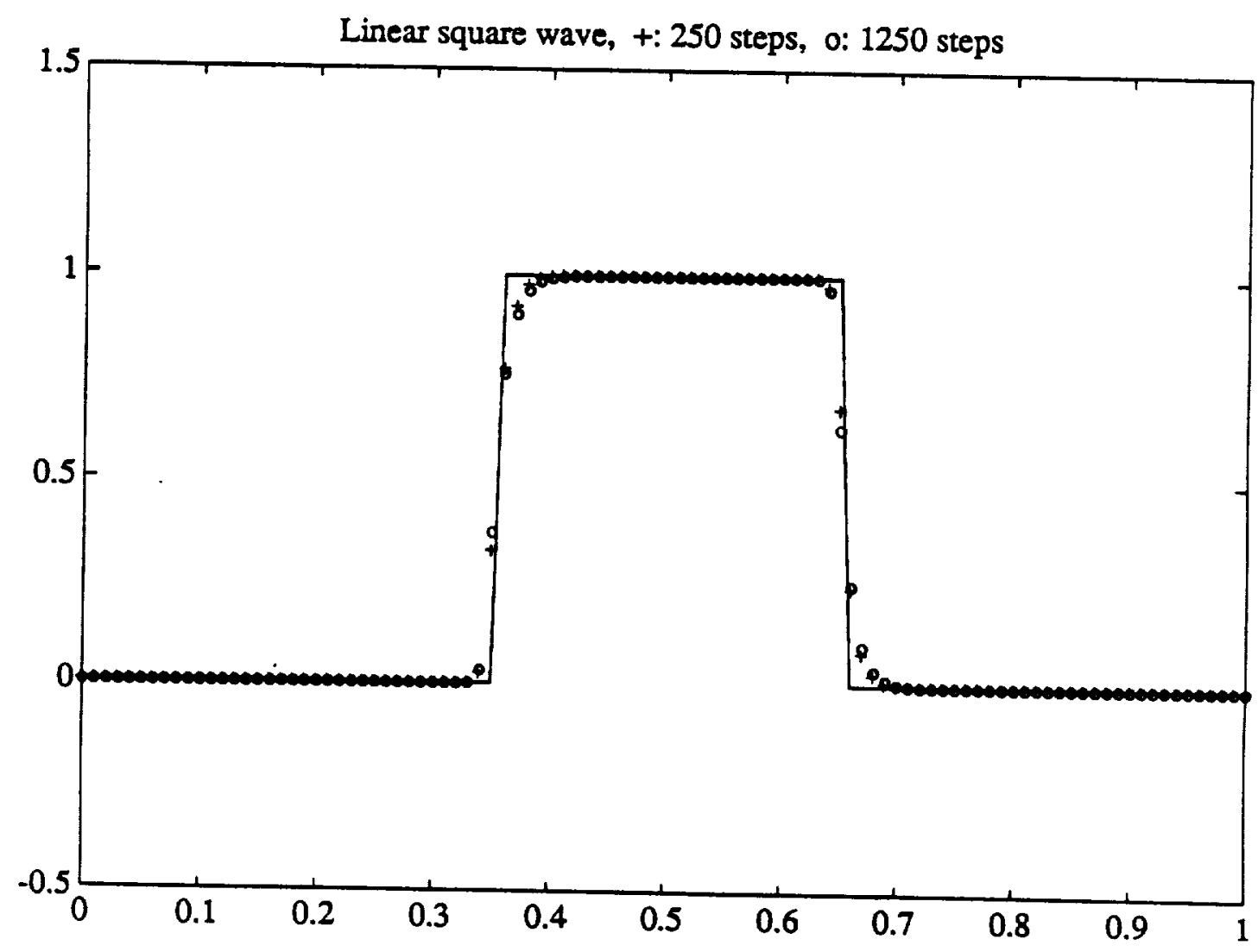

Figure 12: order: $2-1, \quad$ constant alfa $=1.9, \mathrm{CFL}=0.8$ 


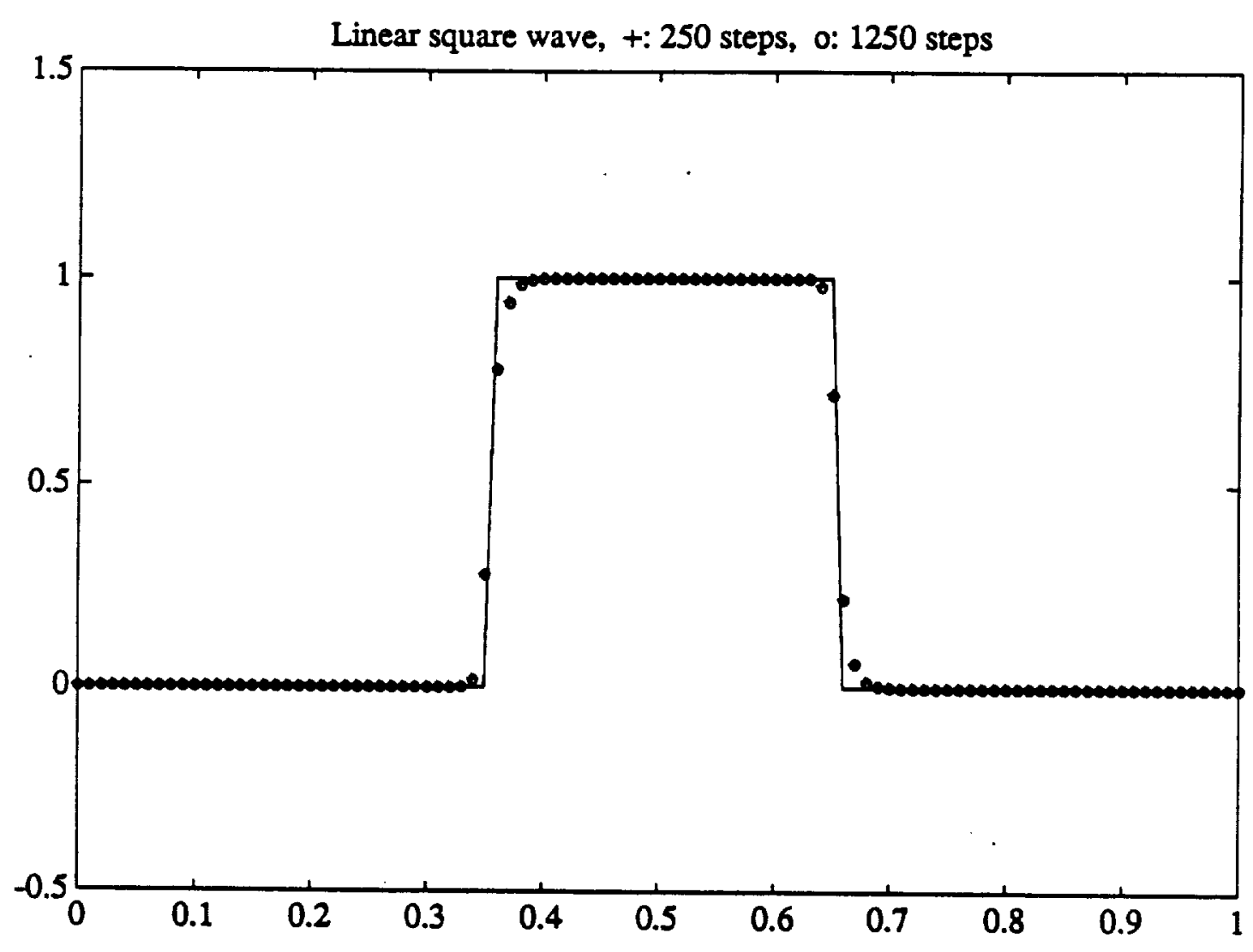

Figure 13: order: $2--1, \quad$ constant alfa $=2.3, C F L=0.8$

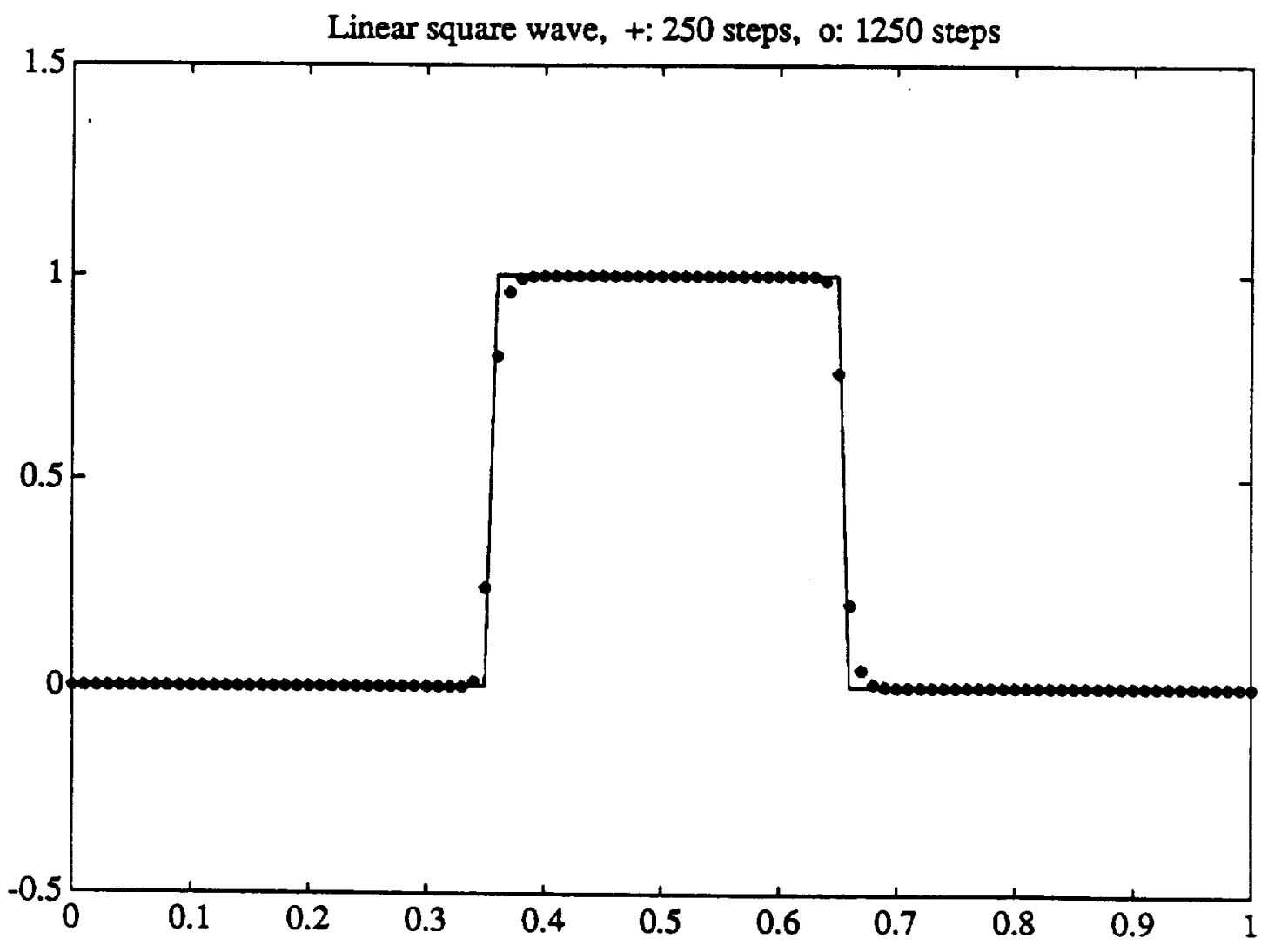

Figure 14: order: $3-0, \quad$ constant alfa $=1.9, C F L=0.8$ 


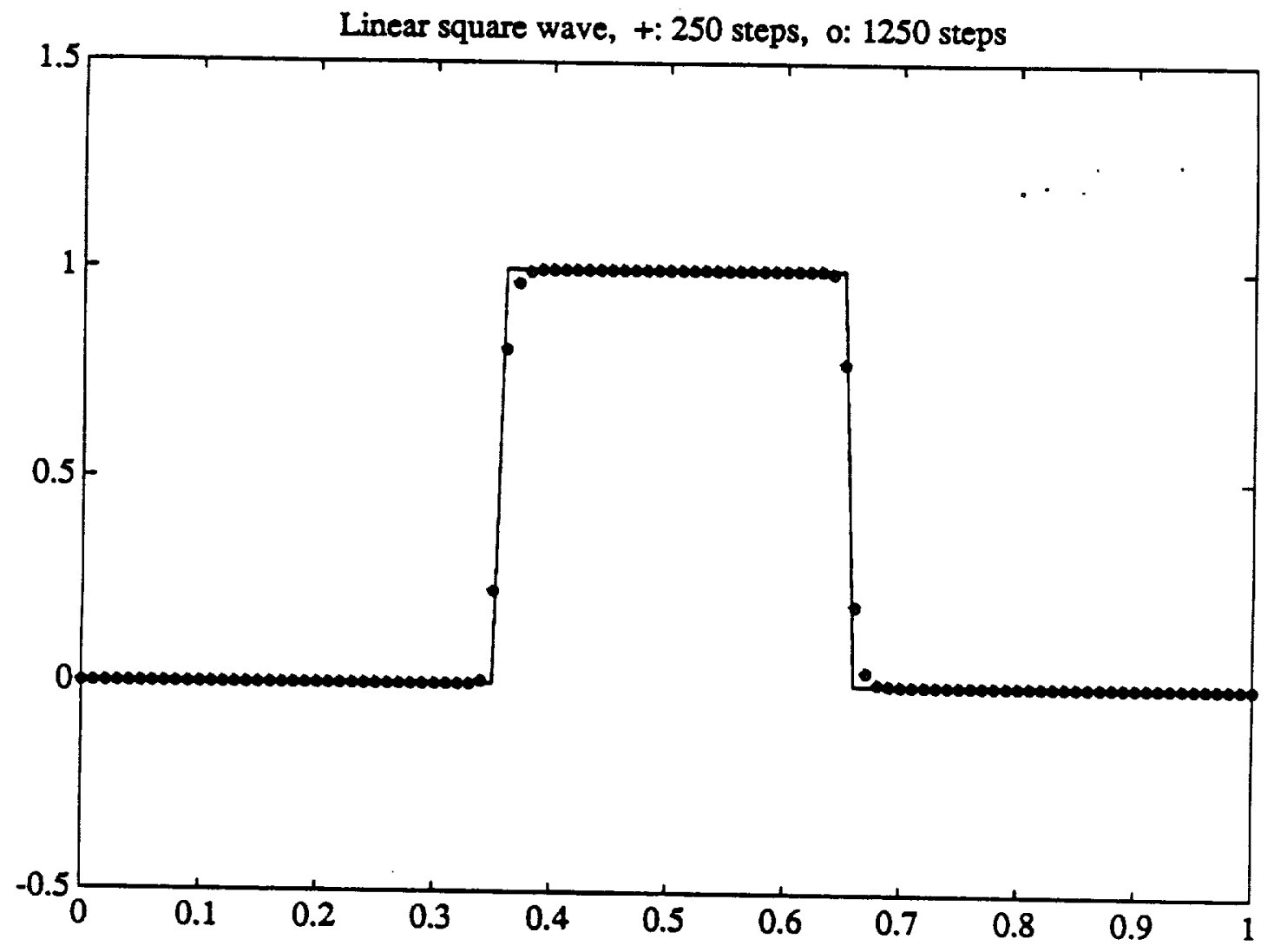

Figure 15: order: $3-1, \quad$ constant alfa $=1.9 . \mathrm{CFI}=\mathrm{n} \mathrm{e}$

Linear square wave, t: 250 steps, o: 1250 steps

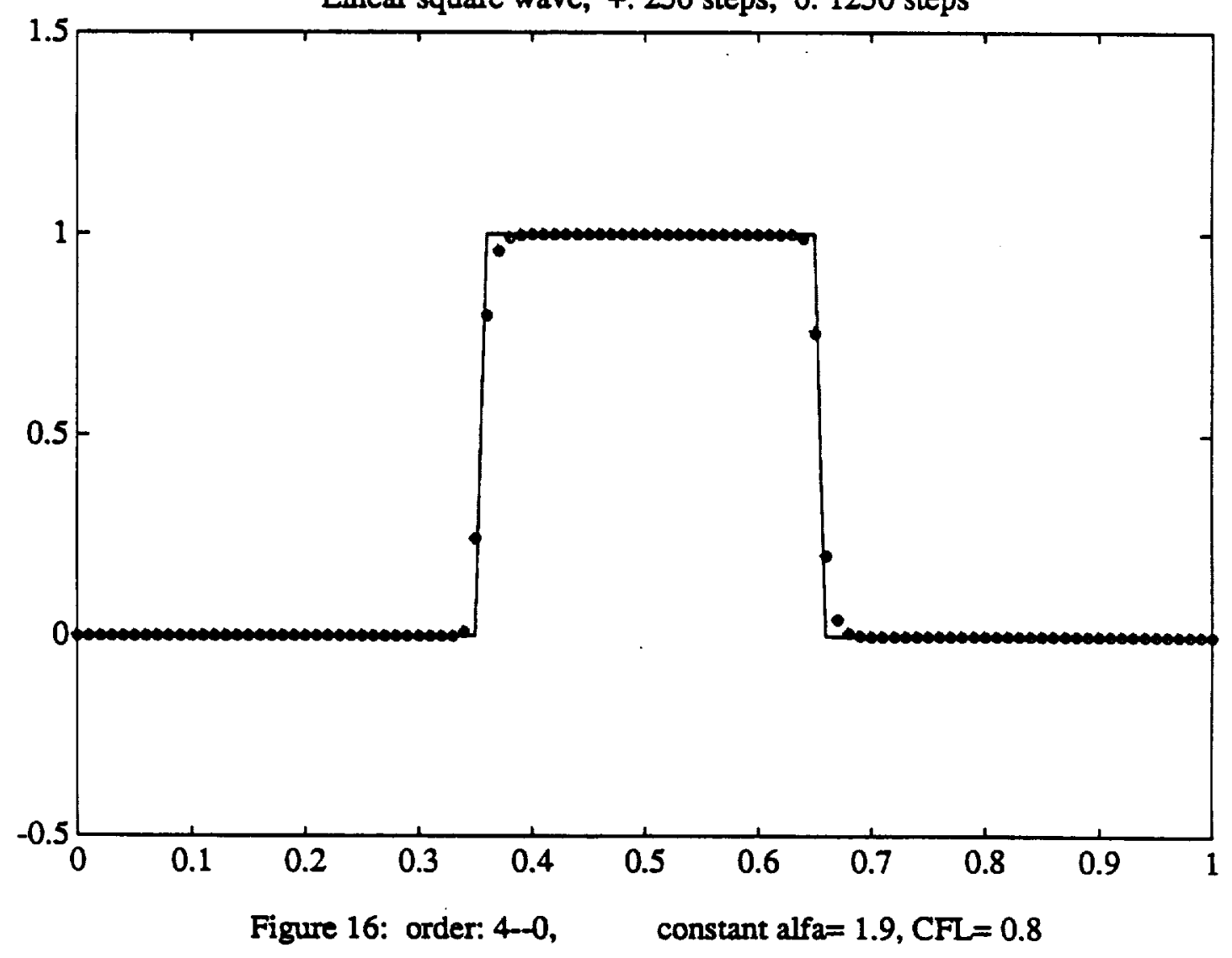




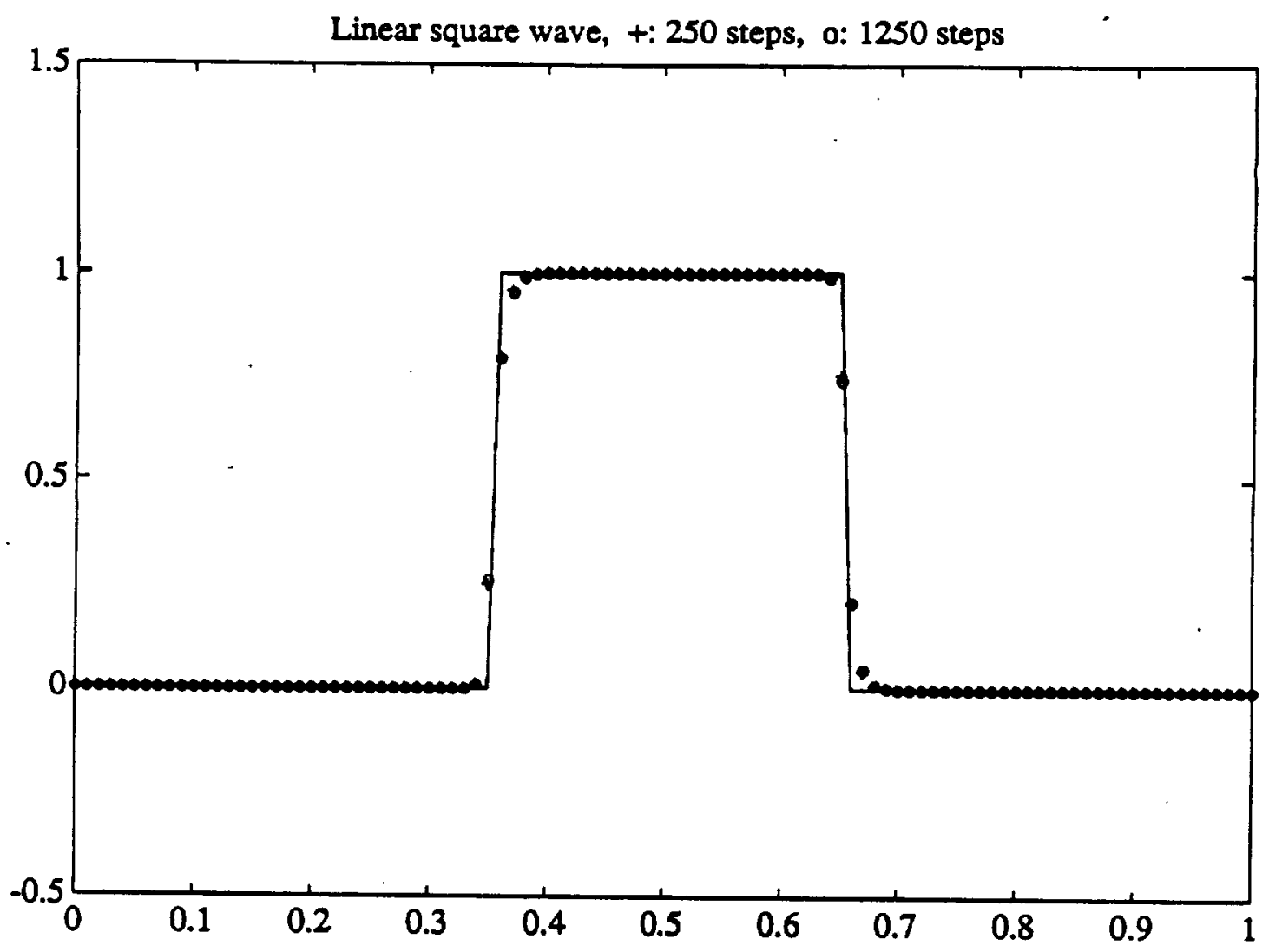

Figure 17: order: 4-1, $\quad$ constant alfa $=1.9, C F L=0.8$

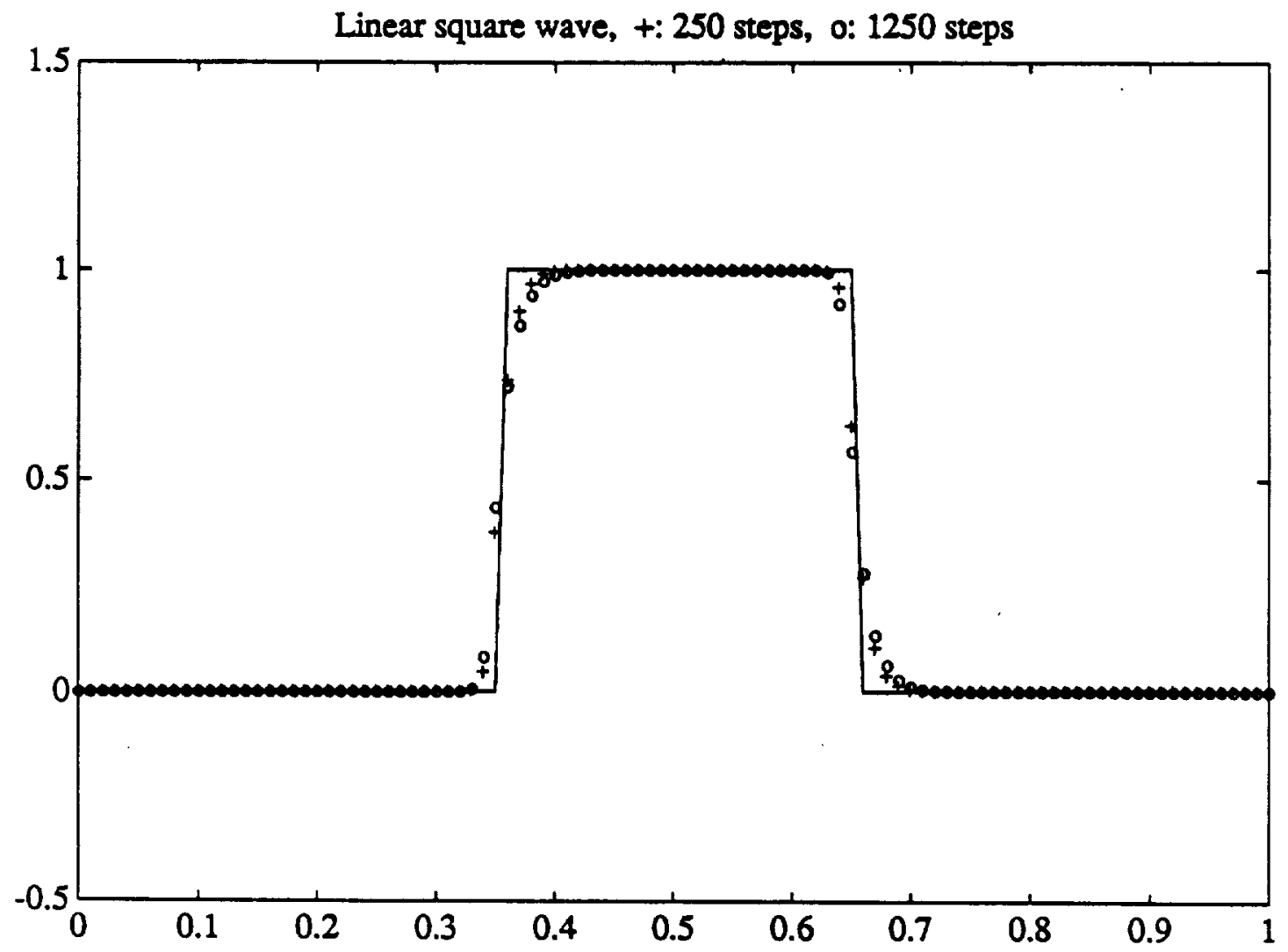

Figure 18: order: 3- $-0, \quad$ constant alfa $=1.0, C F L=0.8$ 


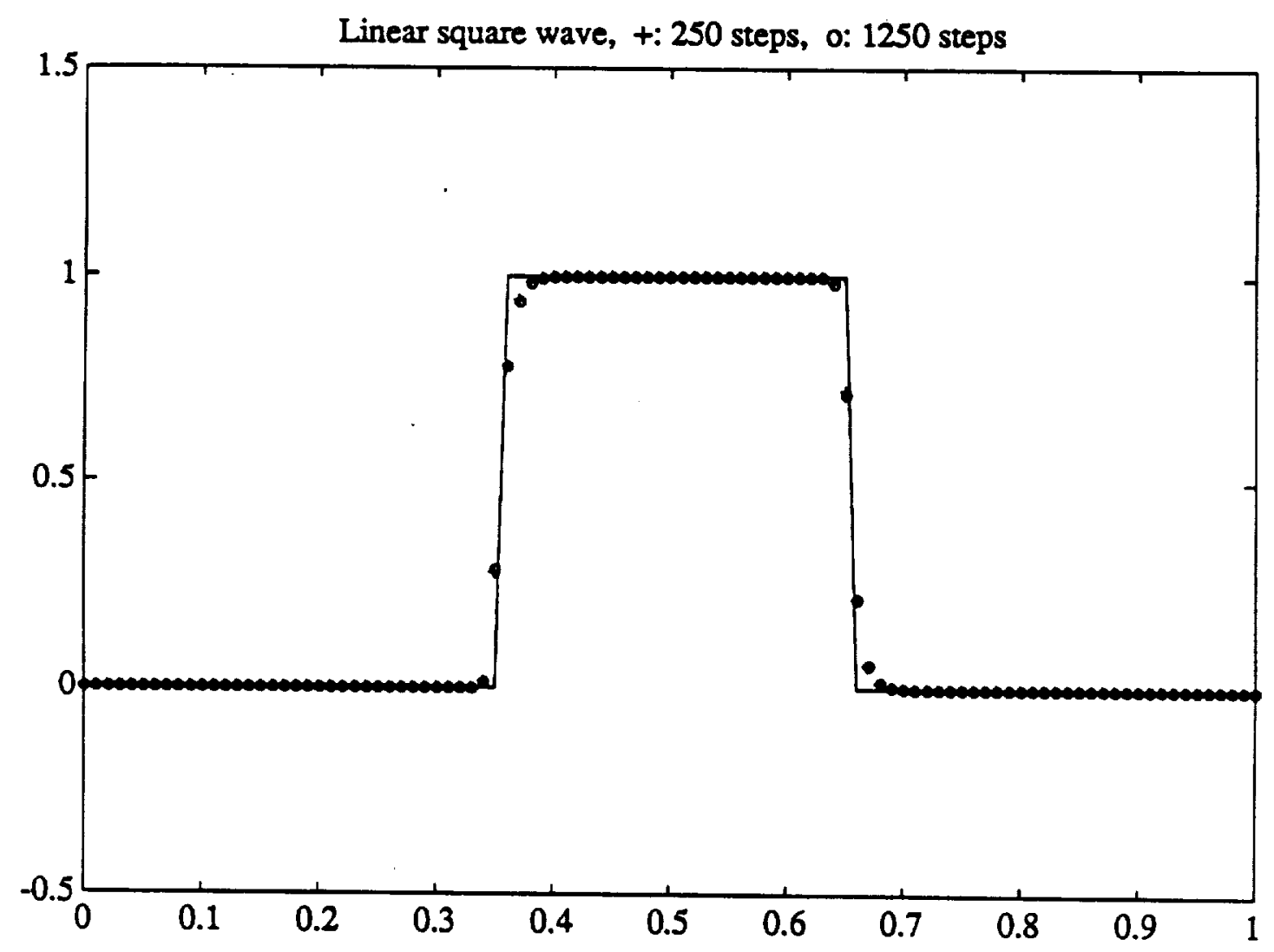

Figure 19: order: $3-0, \quad$ constant alfa $=1.5, C F L=0.8$

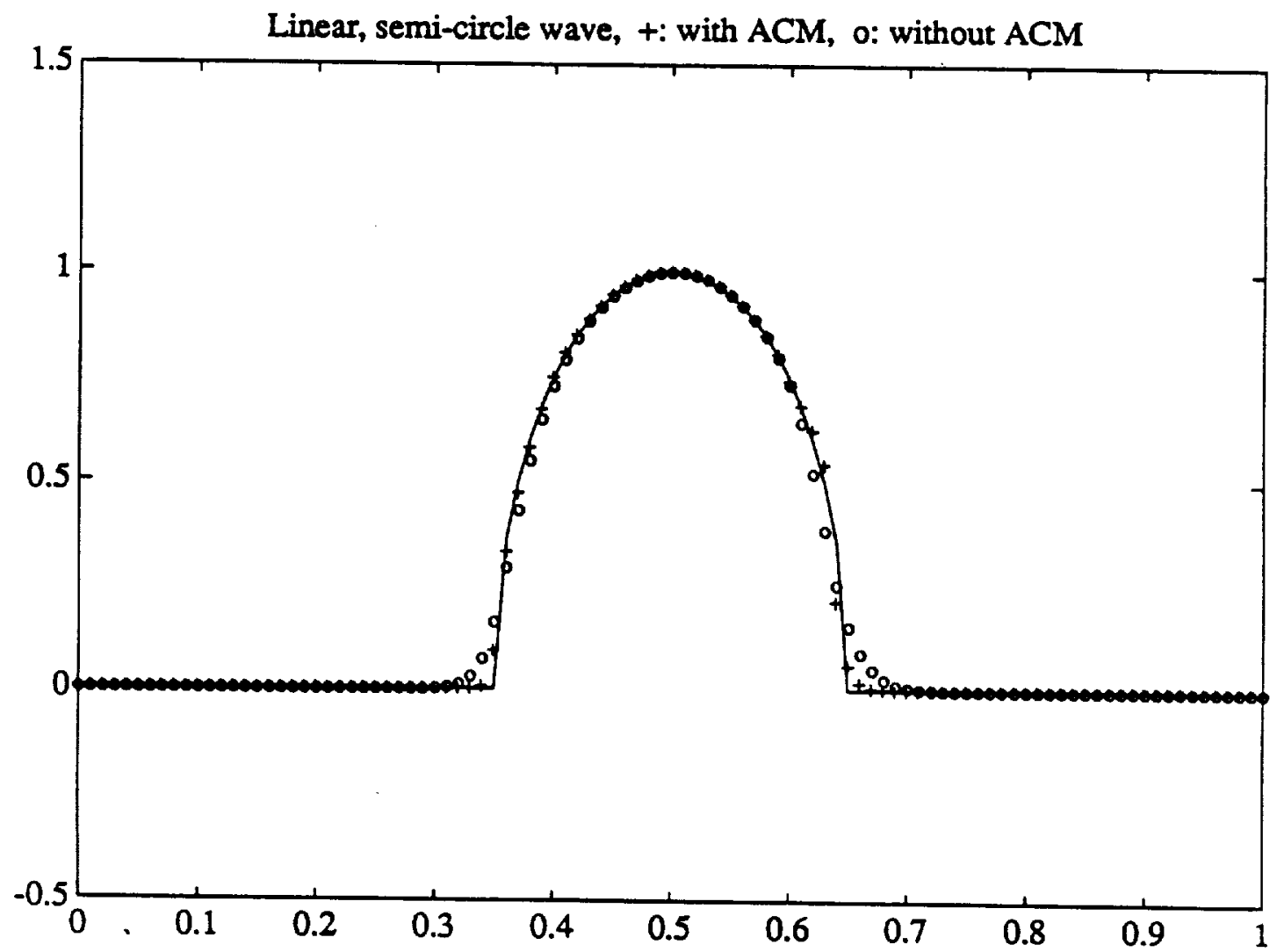

Figure 20: order: $2-1, \quad$ timesteps $=250, C F L=0.8$ 


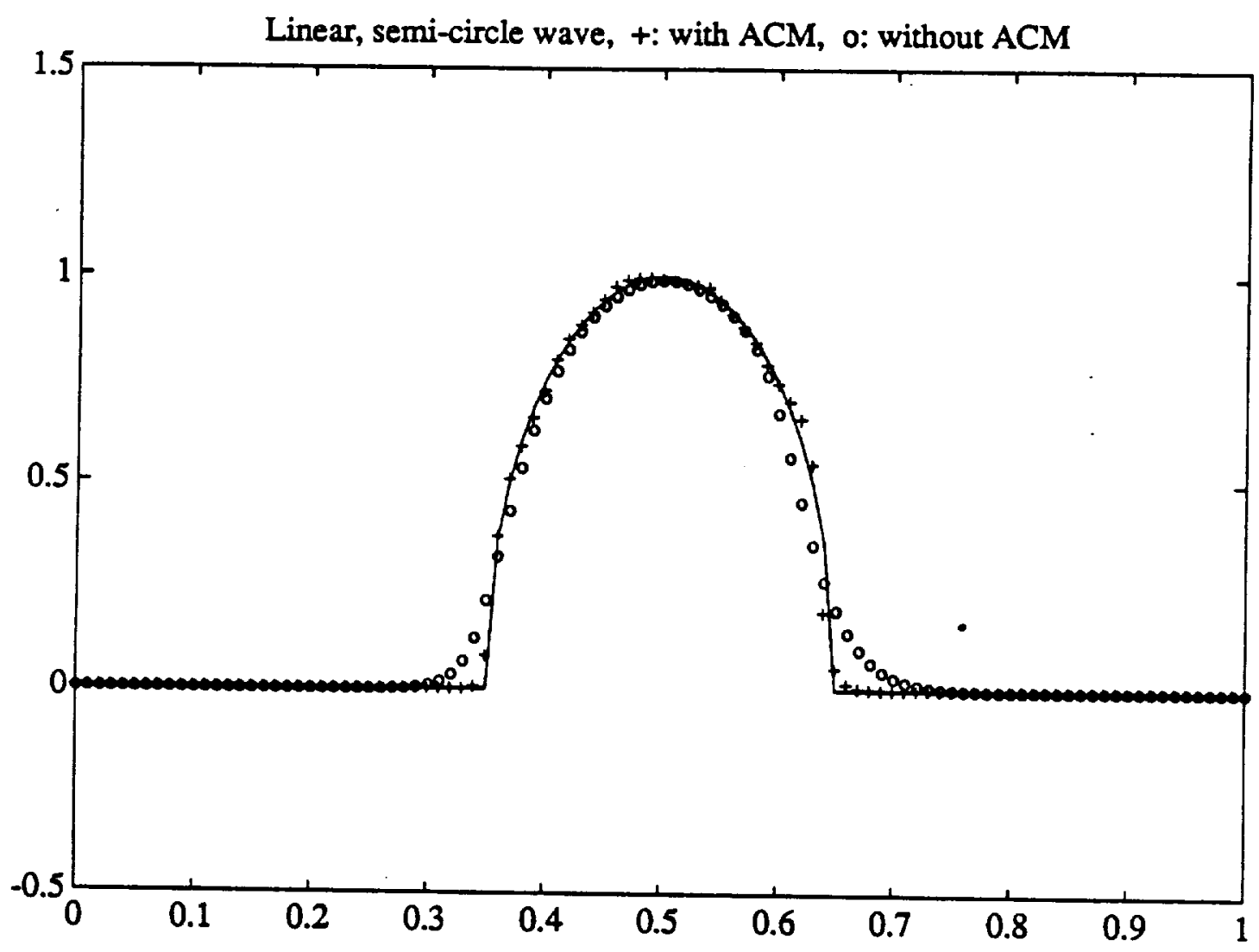

Figure 21: order: 2-1, timesteps $=1250, \mathrm{CFL}=0.8$

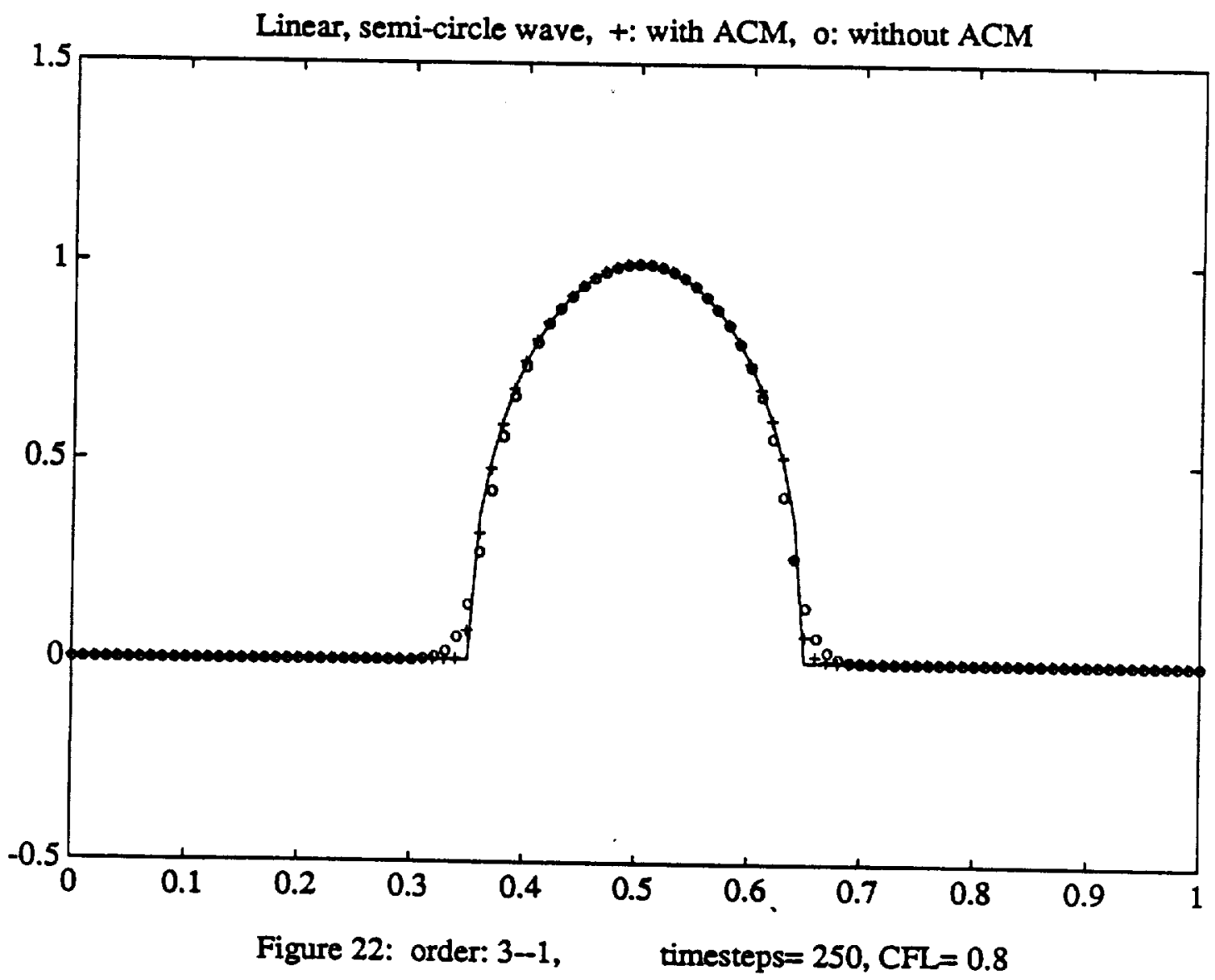




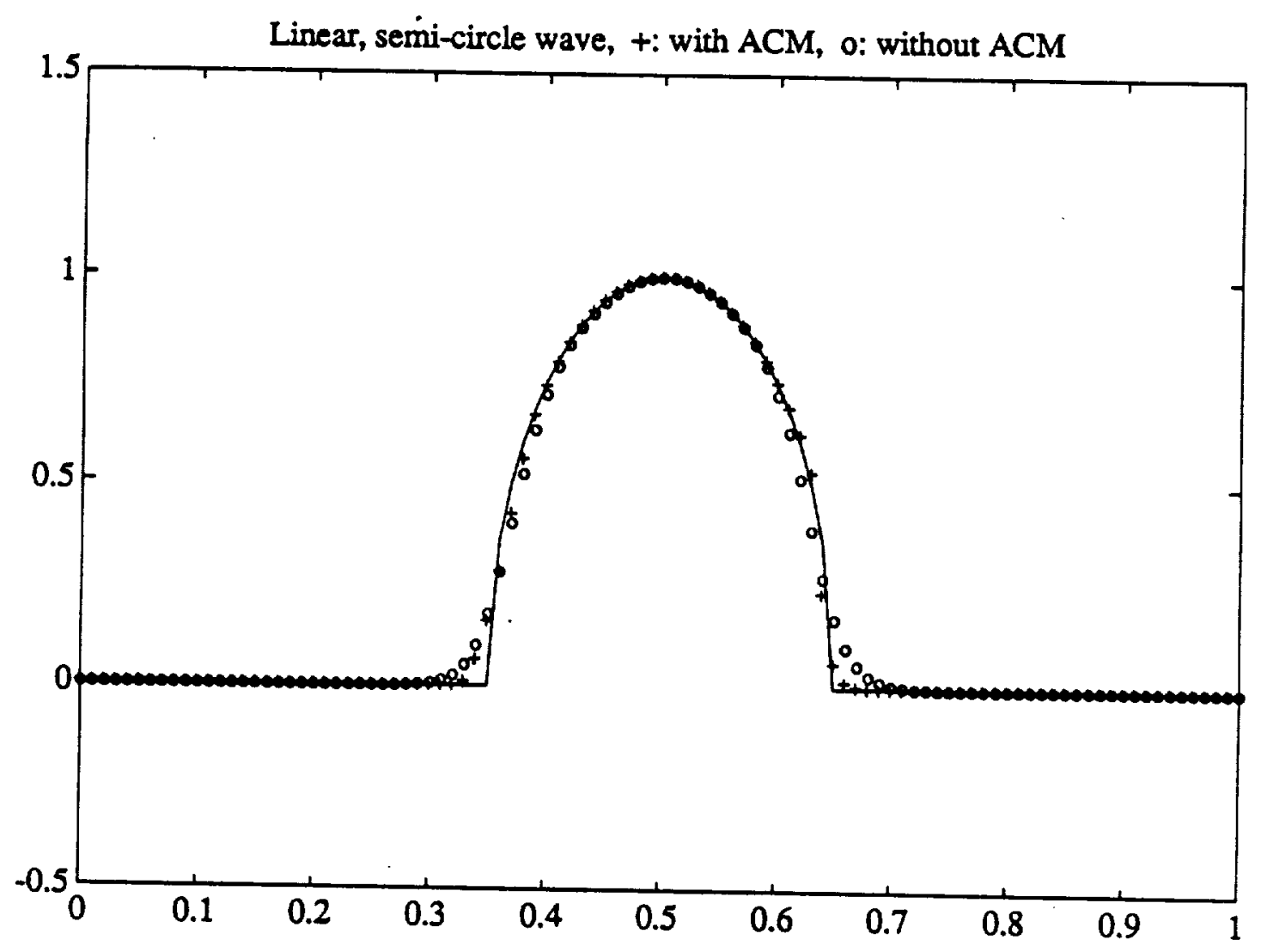

Figure 23: order: 3-1, . timesteps $=1250, C F L=0.8$

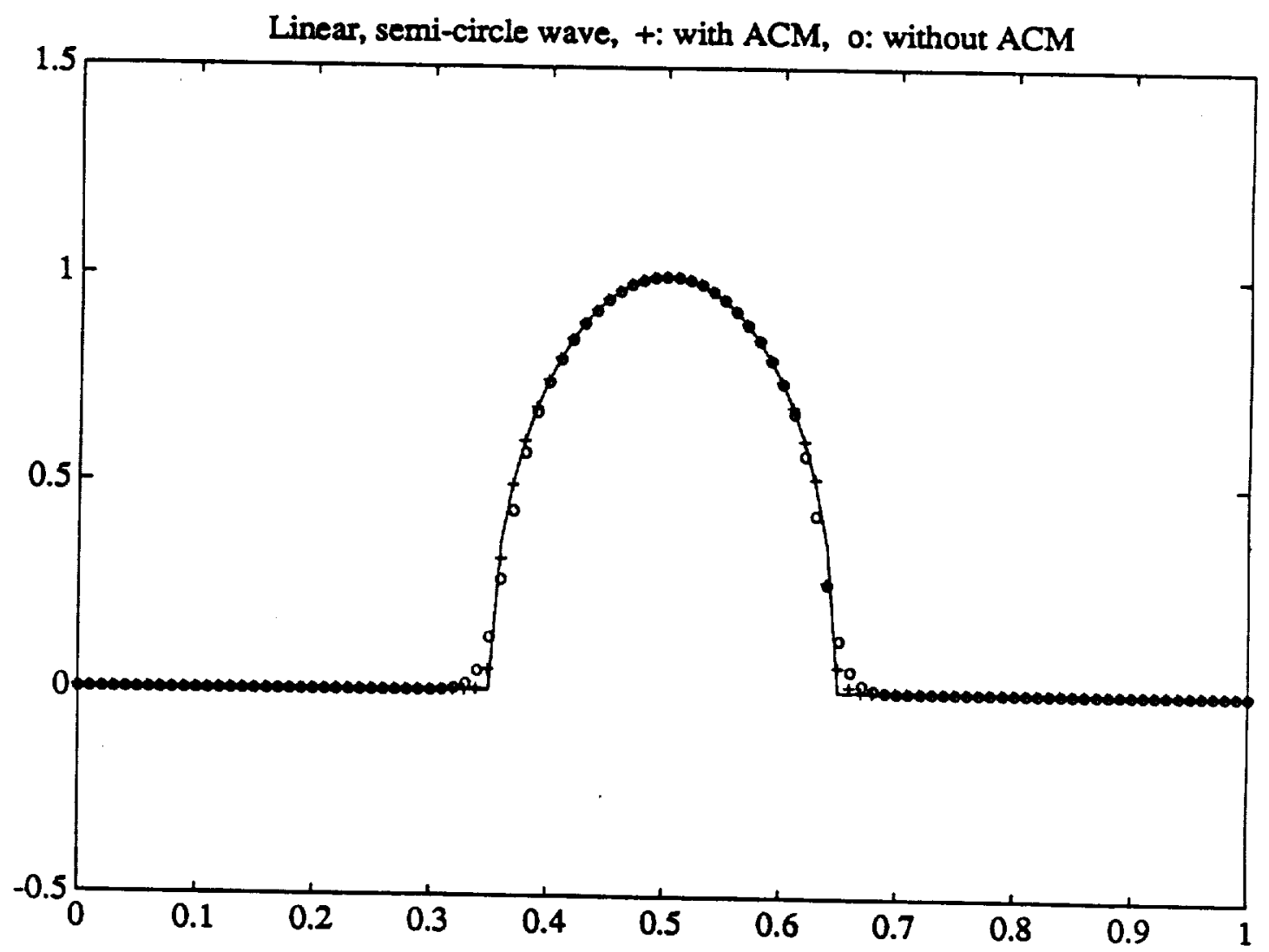

Figure 24: order: $4-1, \quad$ timesteps $=250, C F L=0.8$ 


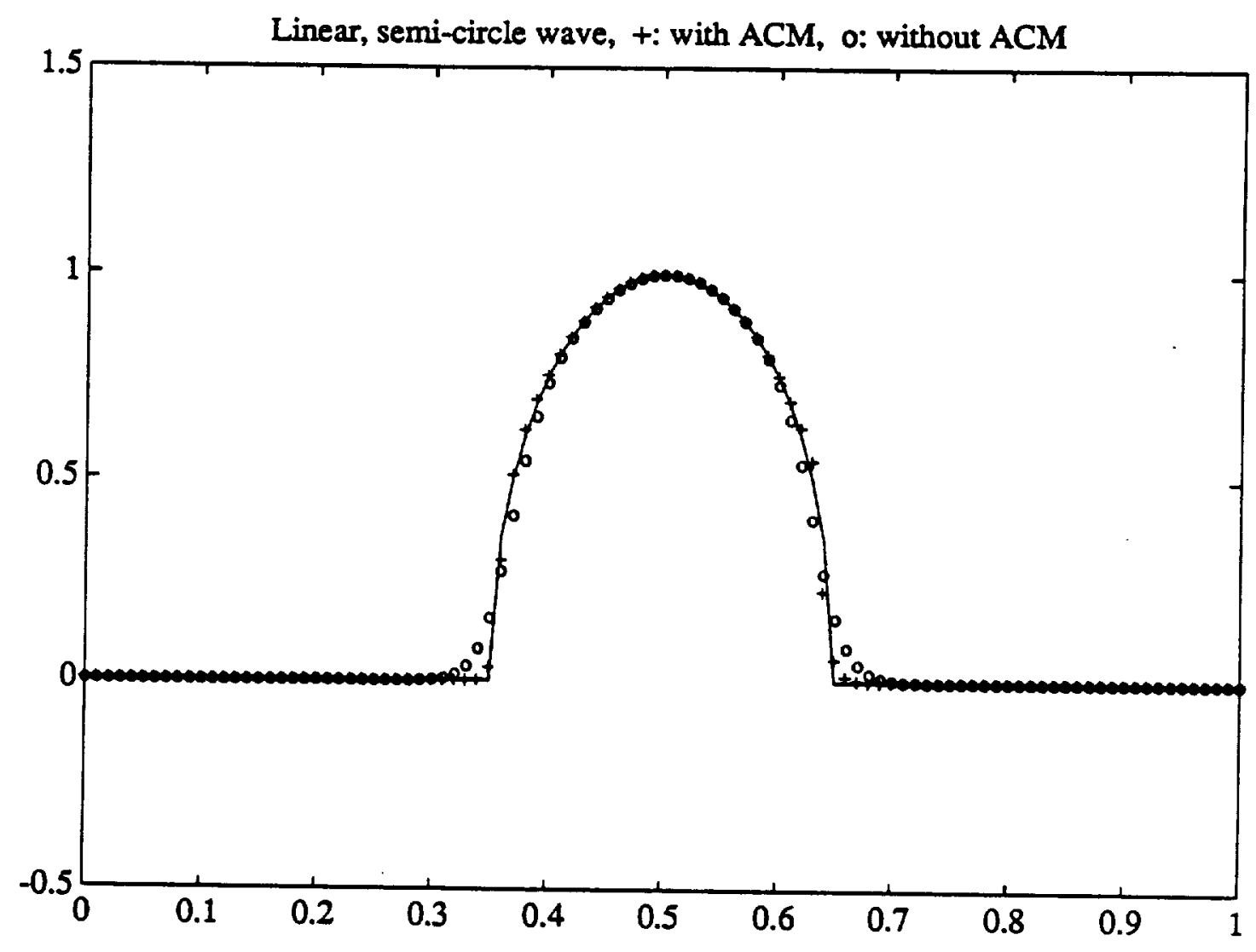

Figure 25: order: $4-1, \quad$ timesteps $=1250, C F L=0.8$

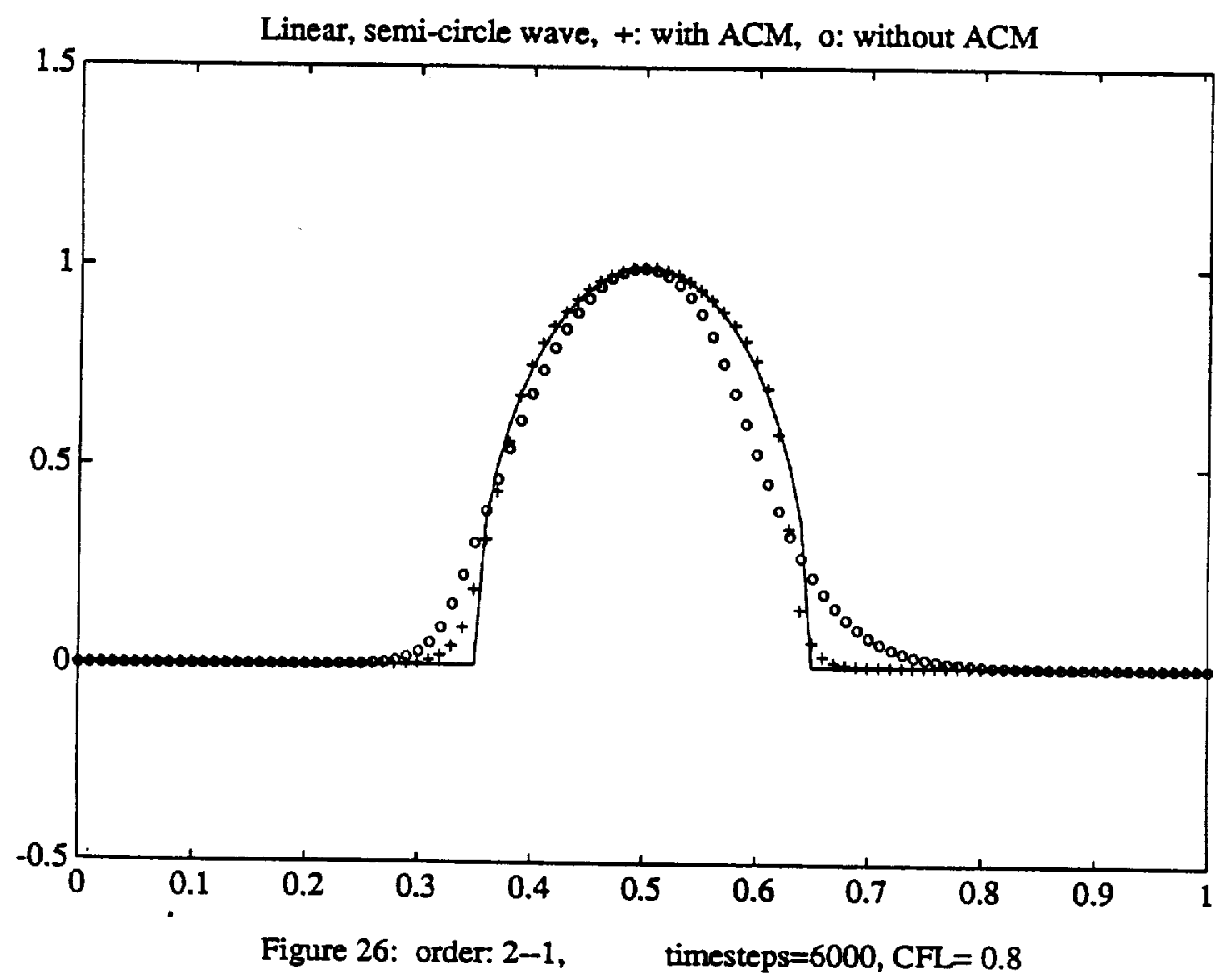



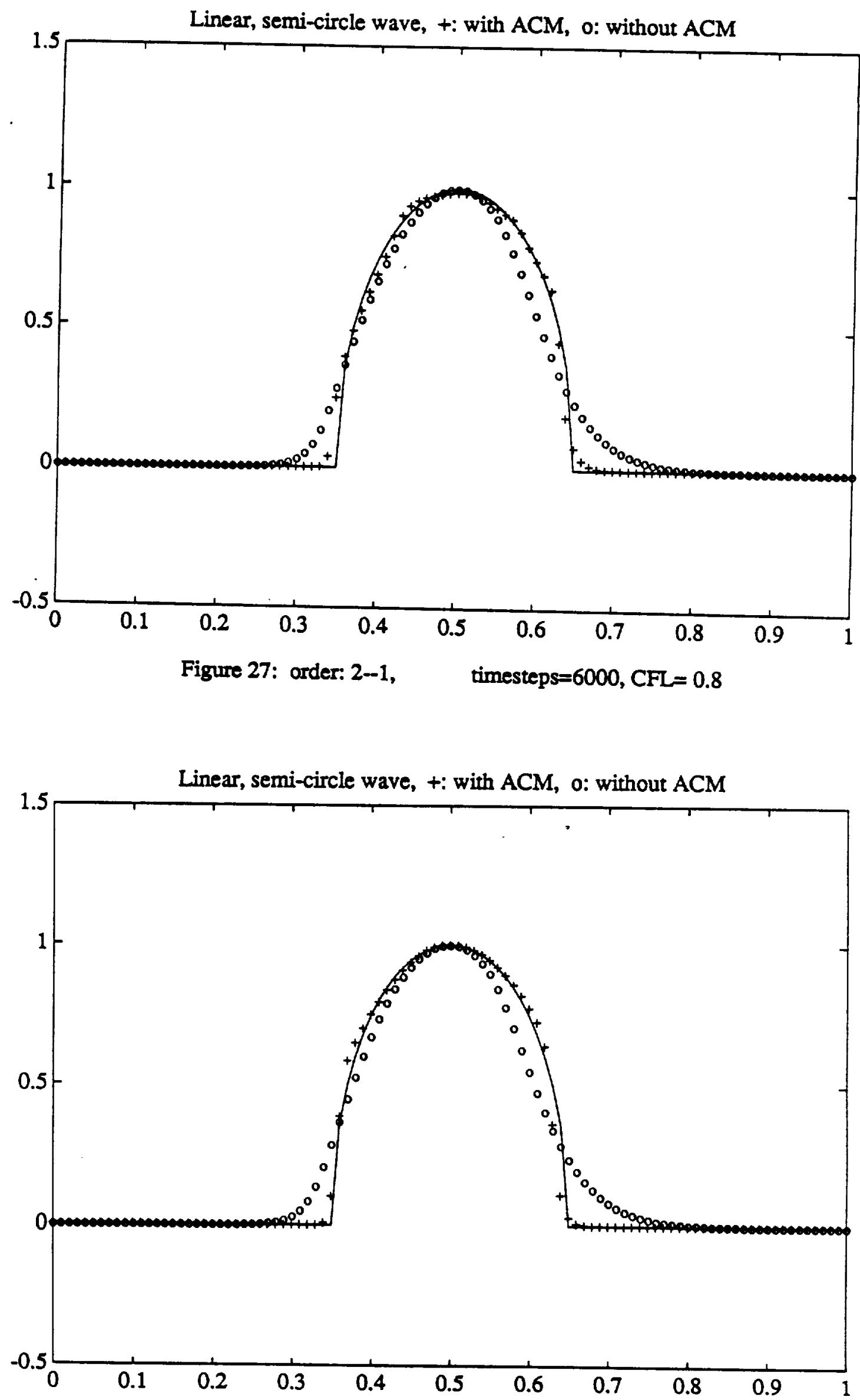

Figure 28: order, 2--1, timesteps $=6000, C F L=0.8$ 

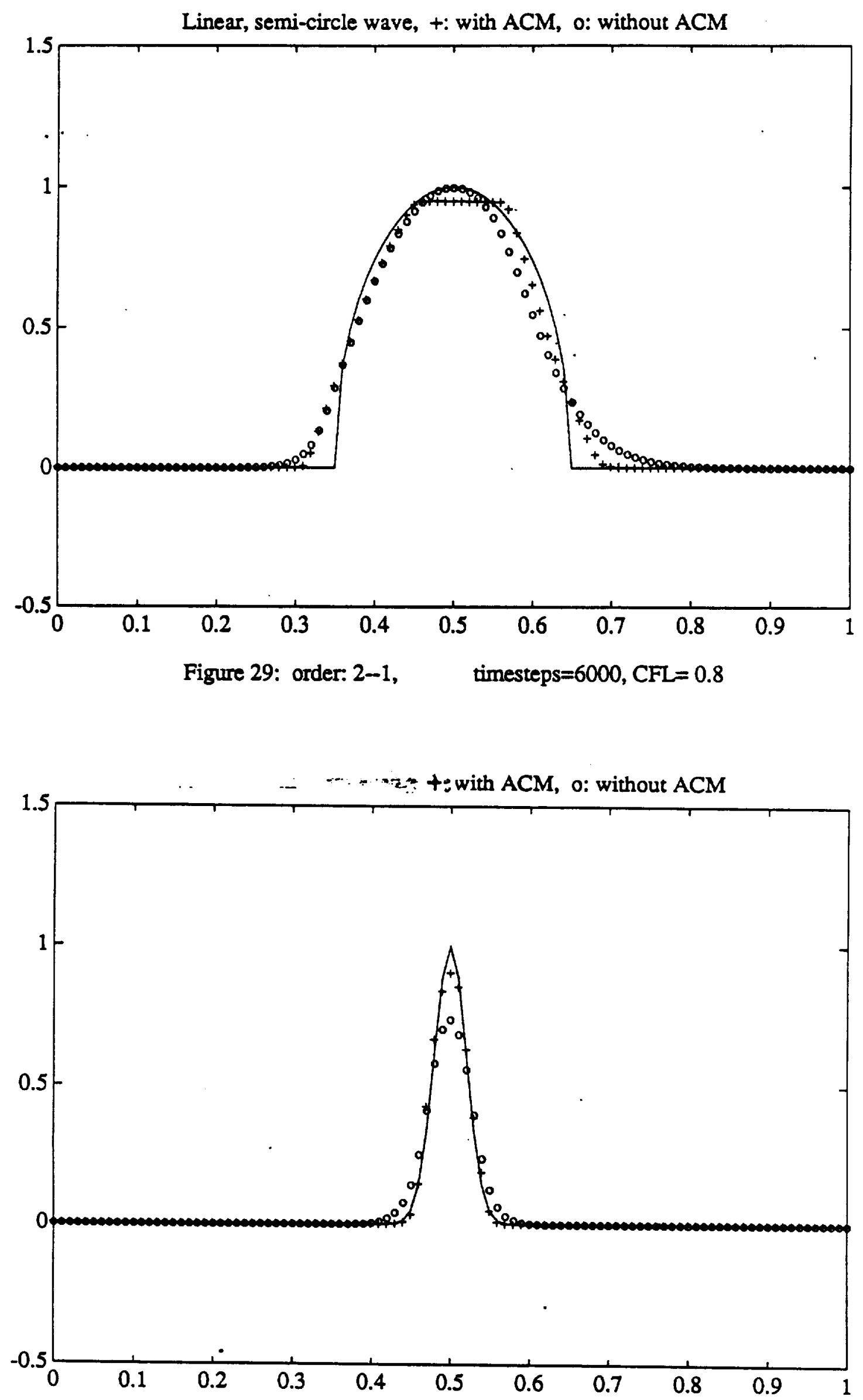

Figure 30: order: 3-0, timesteps $=600, C F L=0.1$ 


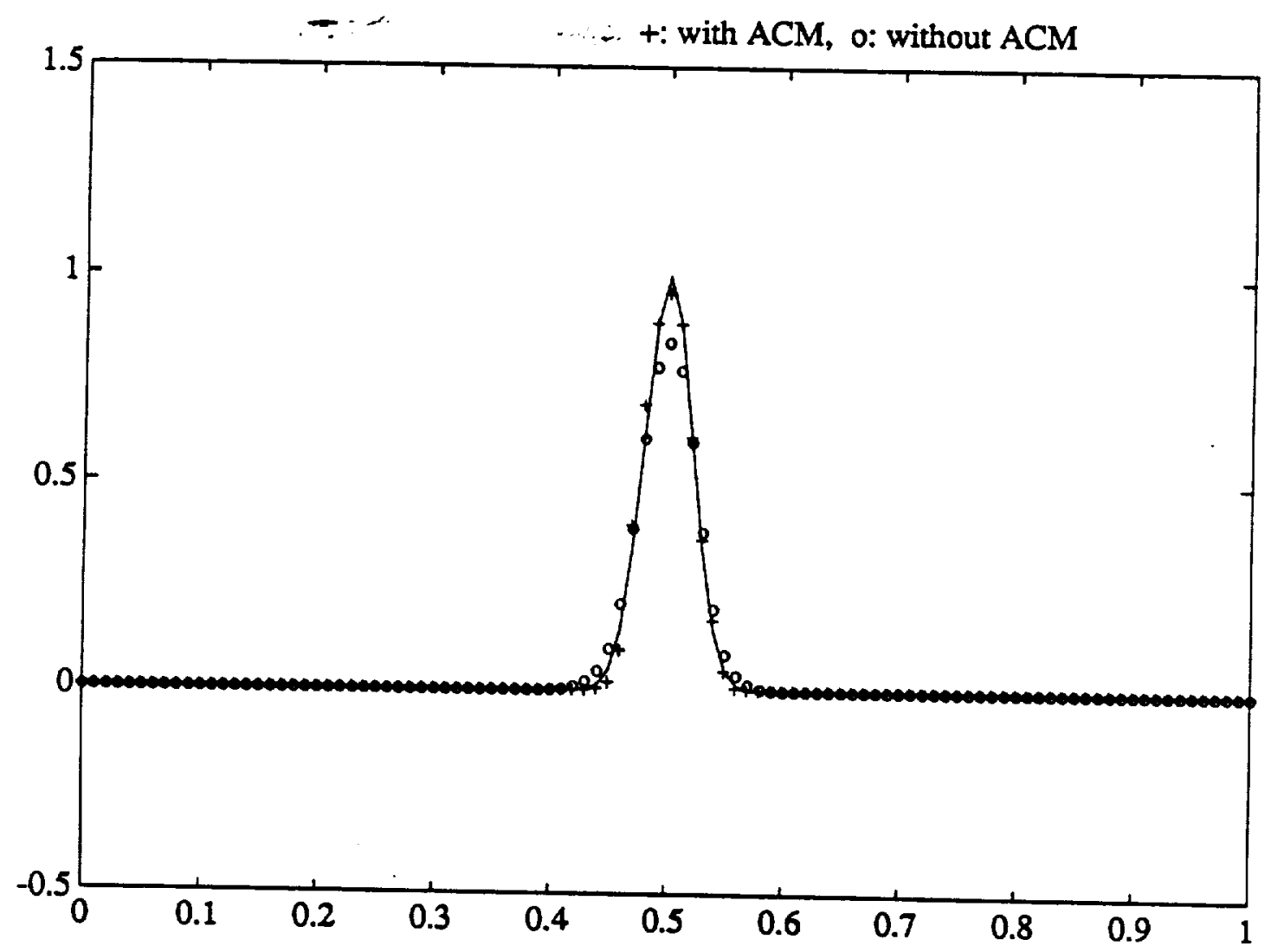

Figure 31: order: 4-0, timesteps $=600, C F L=0.1$

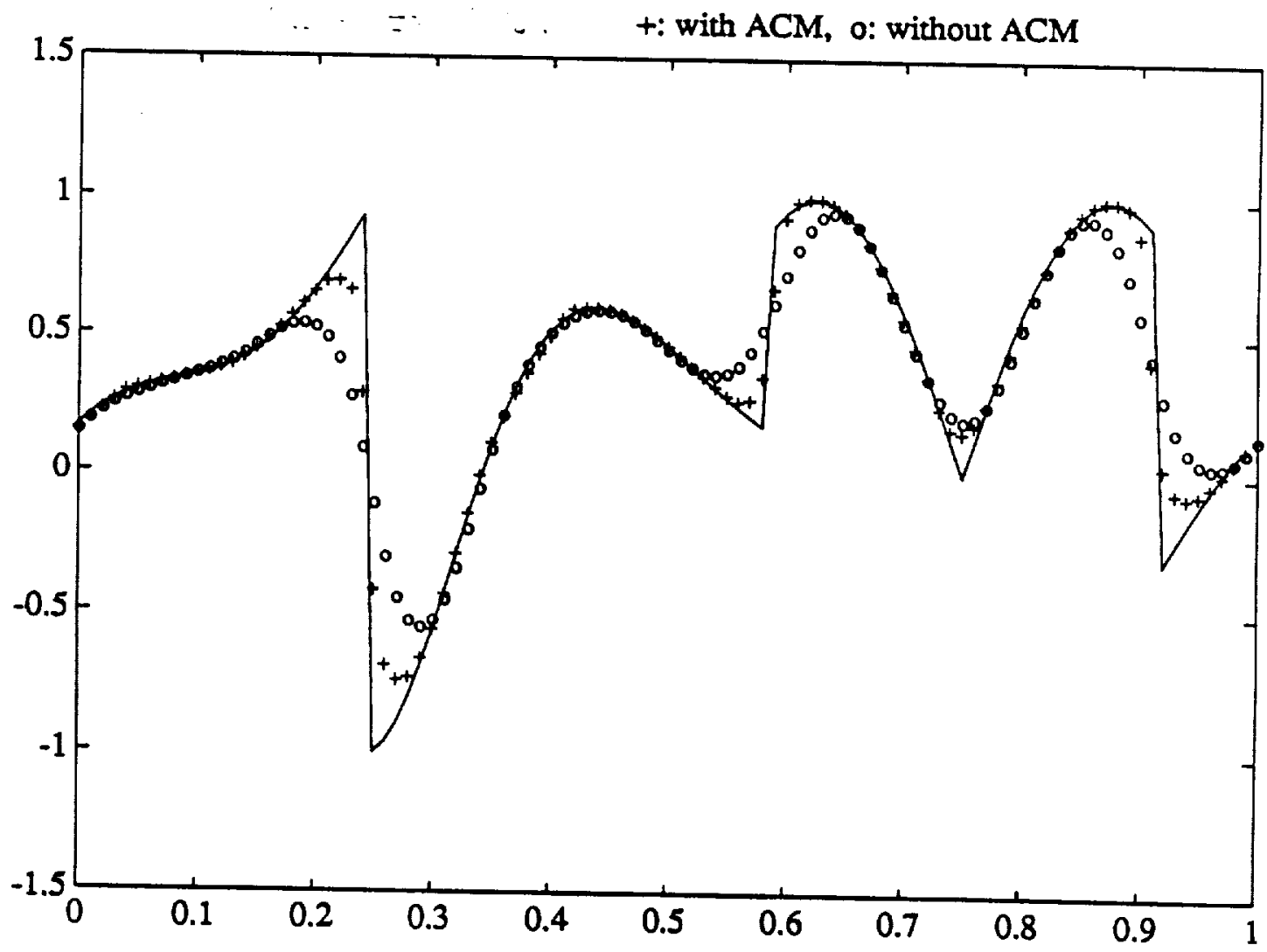

Figure 32: order: $3--0, \quad$ timesteps $=1000, C F L=0.8$ 

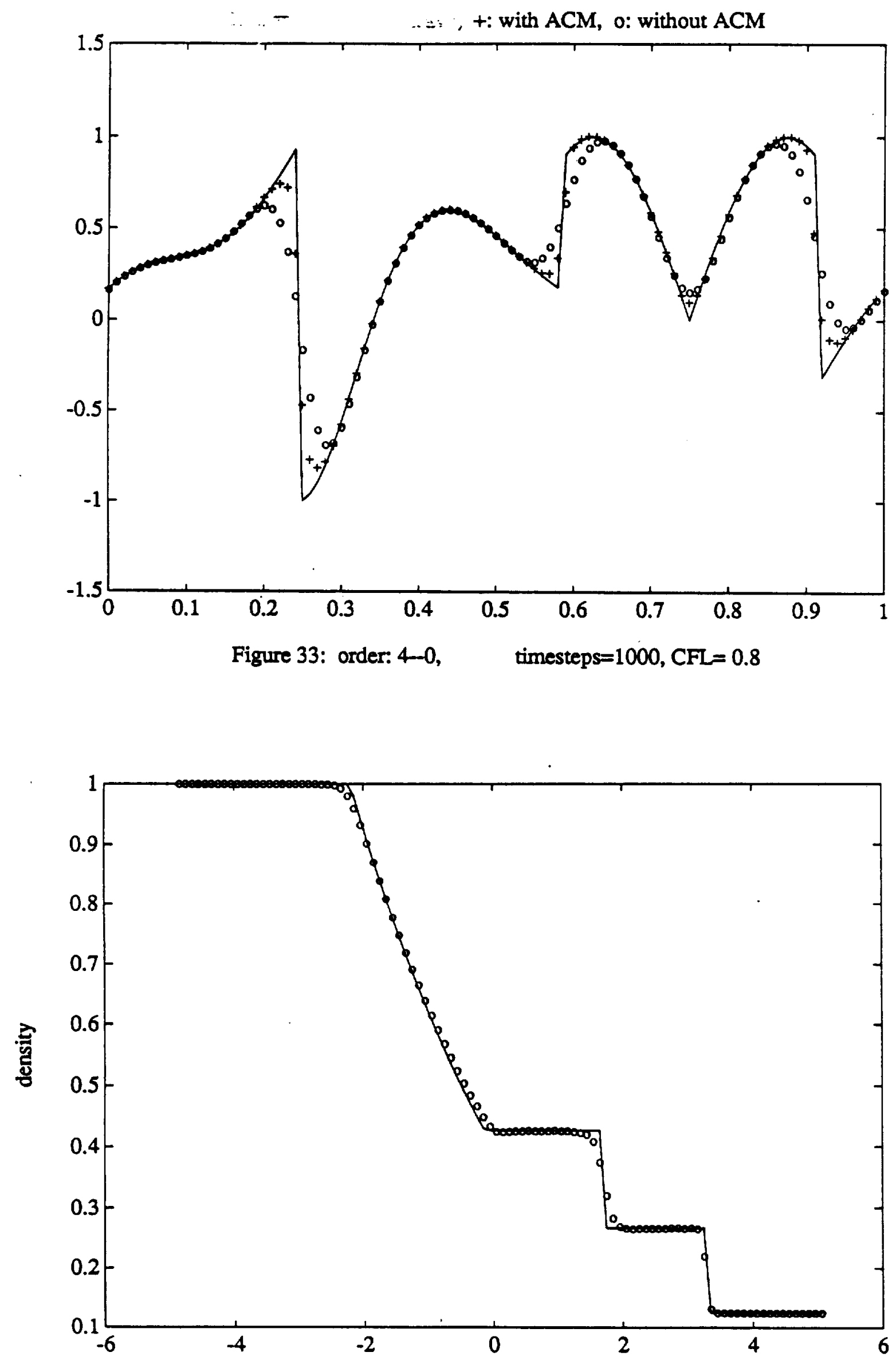

Figure 34.1: Sod problem, "4-th order" ENO without SM 


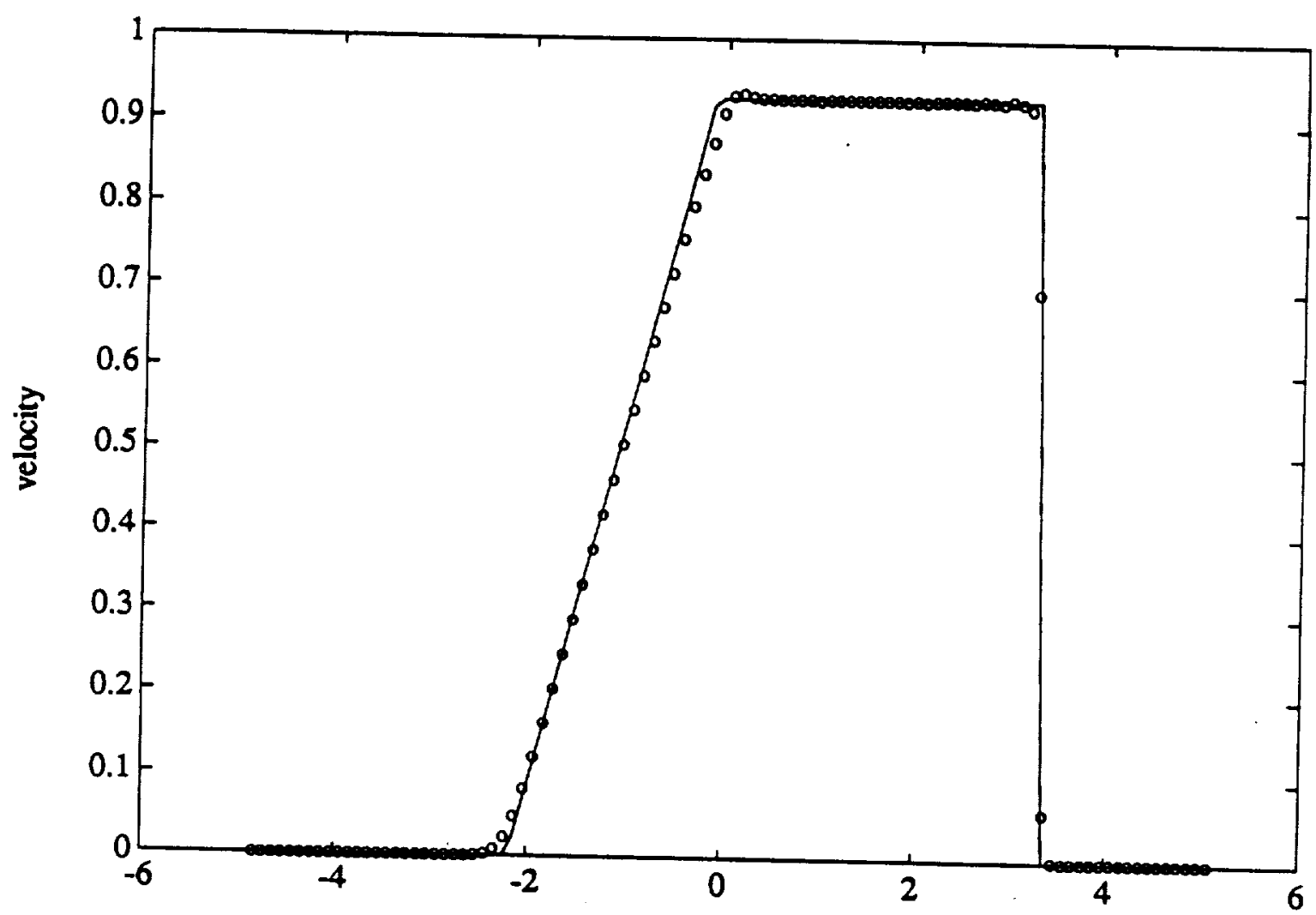

Figure 34.2: Sod problem, "4-th order" ENO without SM

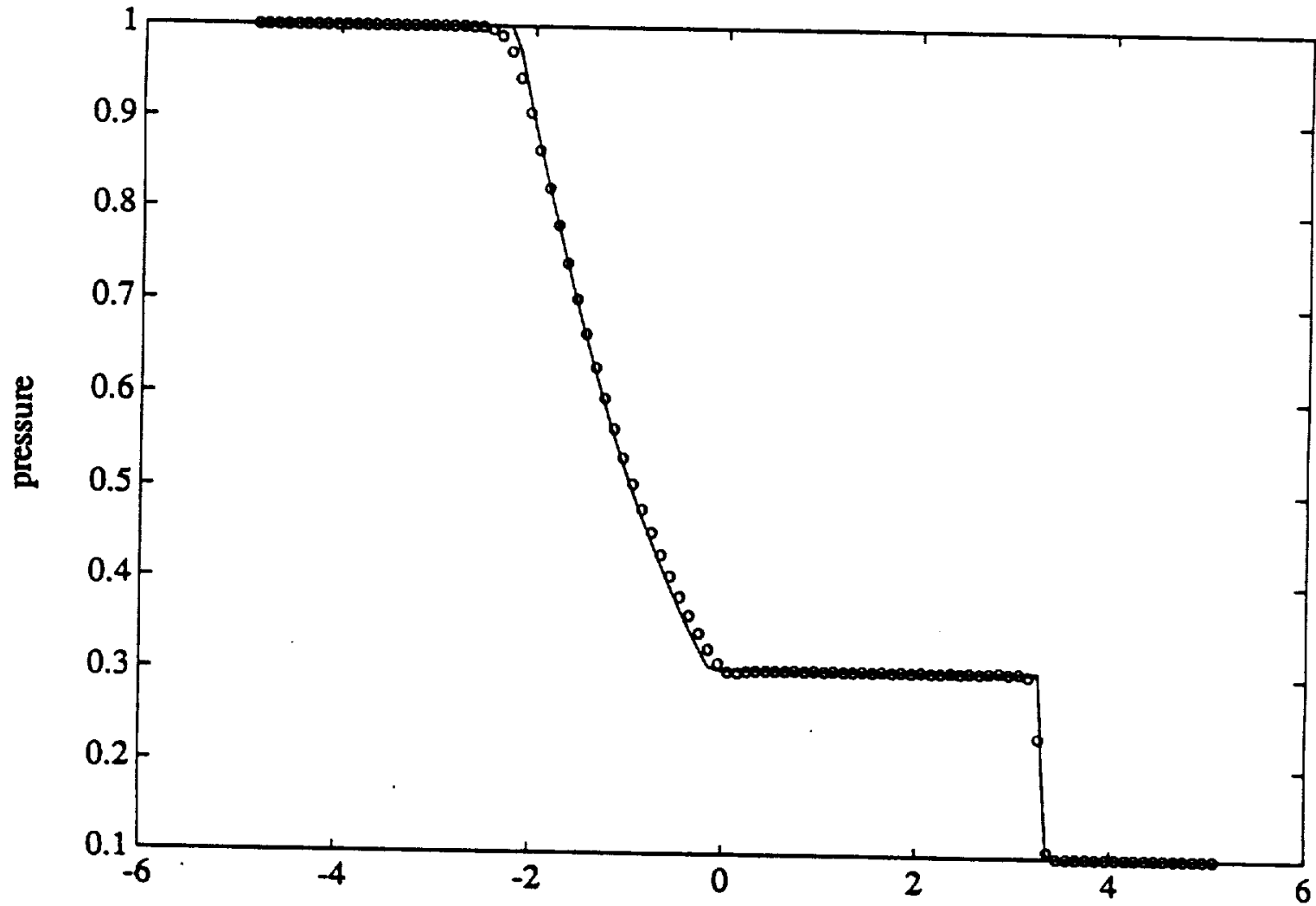

Figure 34.3: Sod problem, "4-th order" ENO without SM 


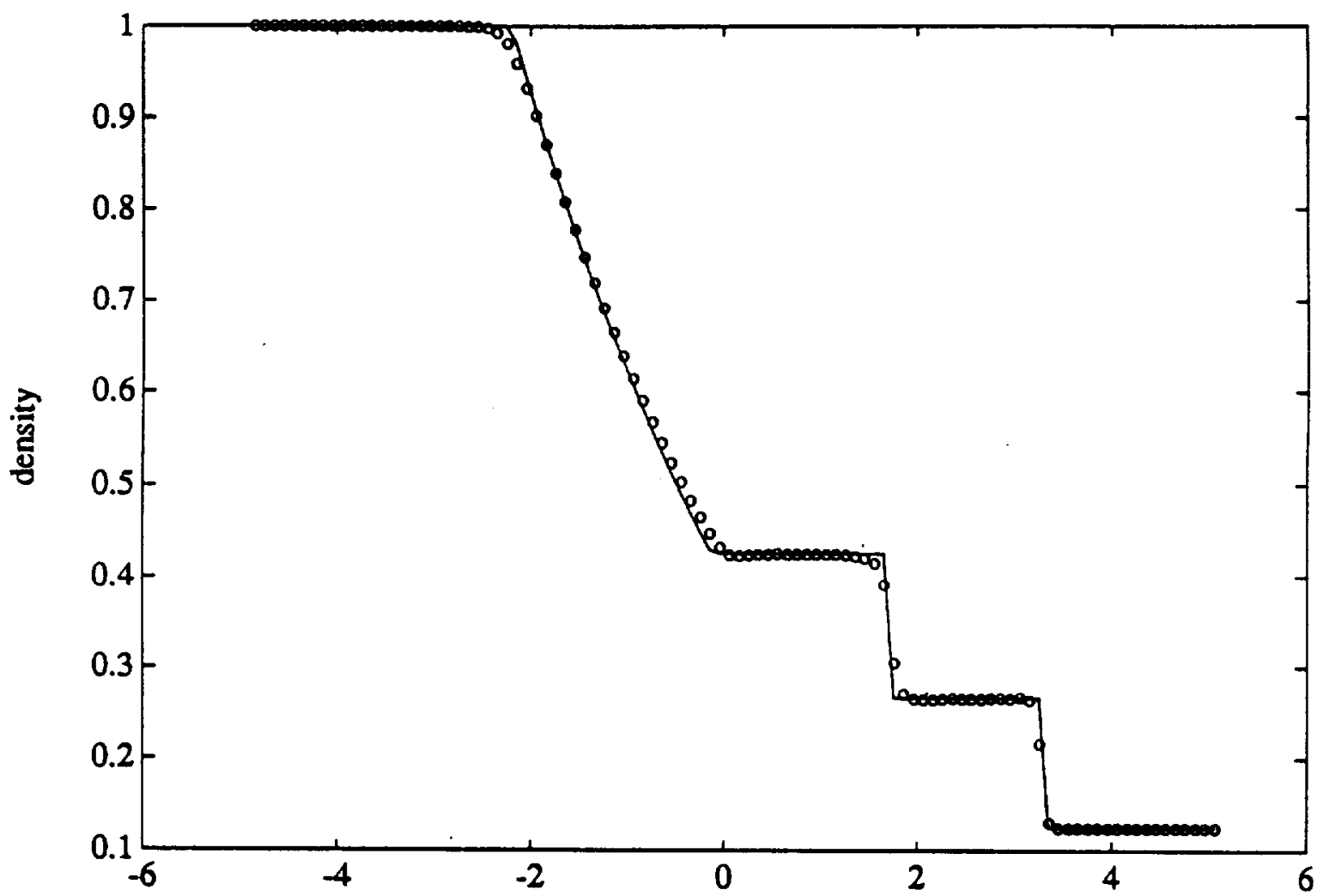

Figure 35.1: Sod problem, "4-th order" ENO with SM

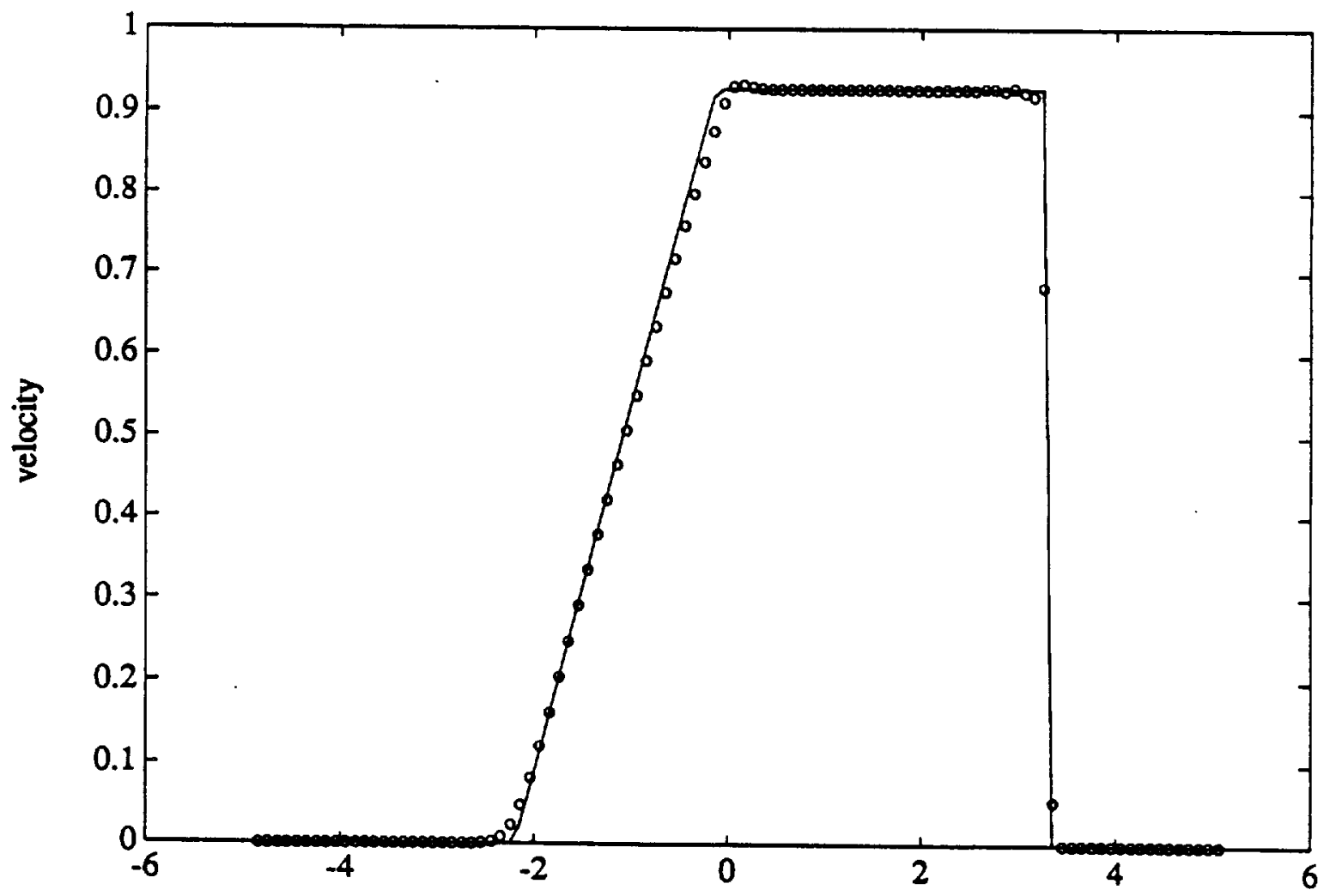

Figure 35.2: Sod problem, "4-th order" ENO with SM 


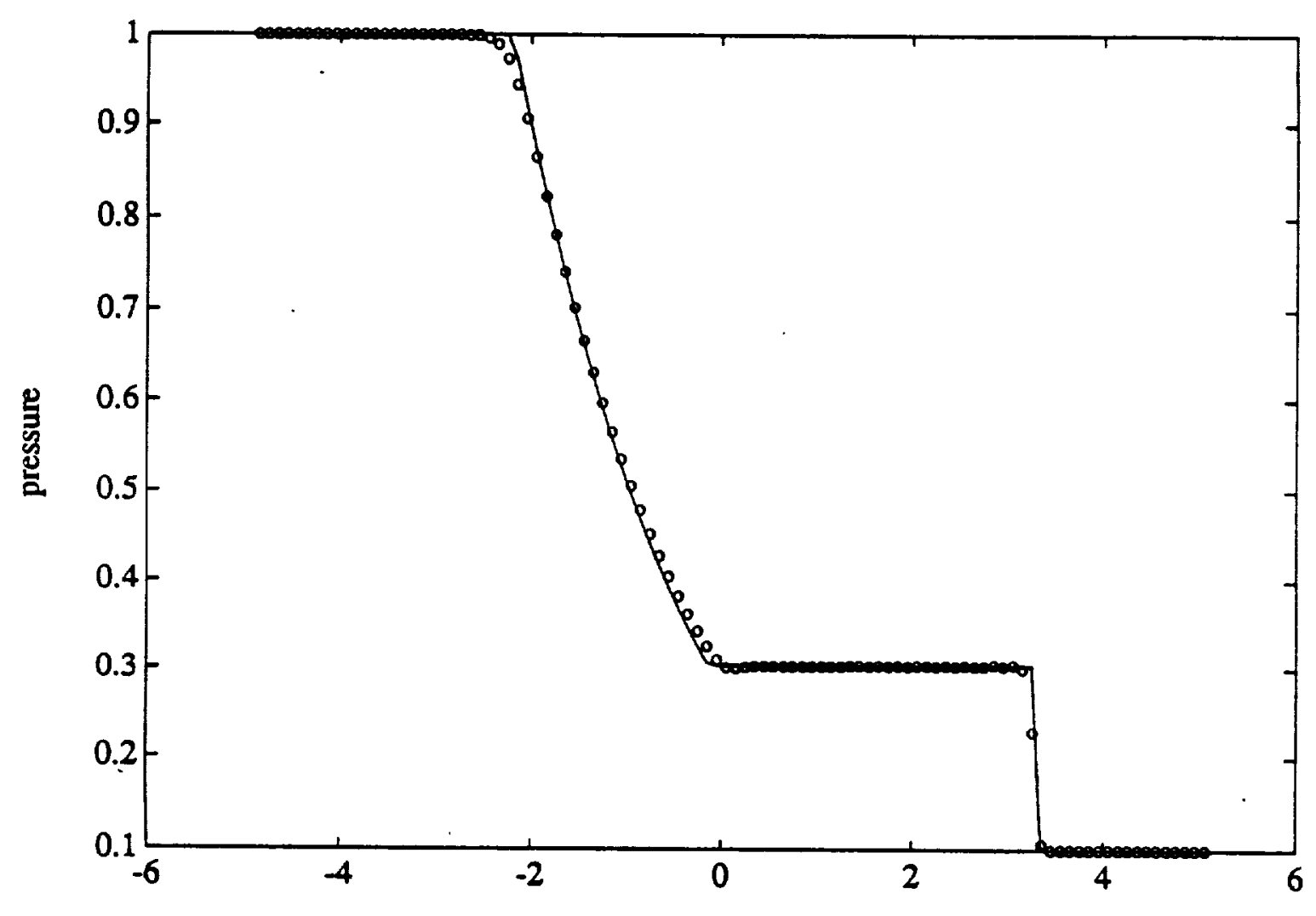

Figure 35.3: Snd nmhlem. "4-th nrder" with SM

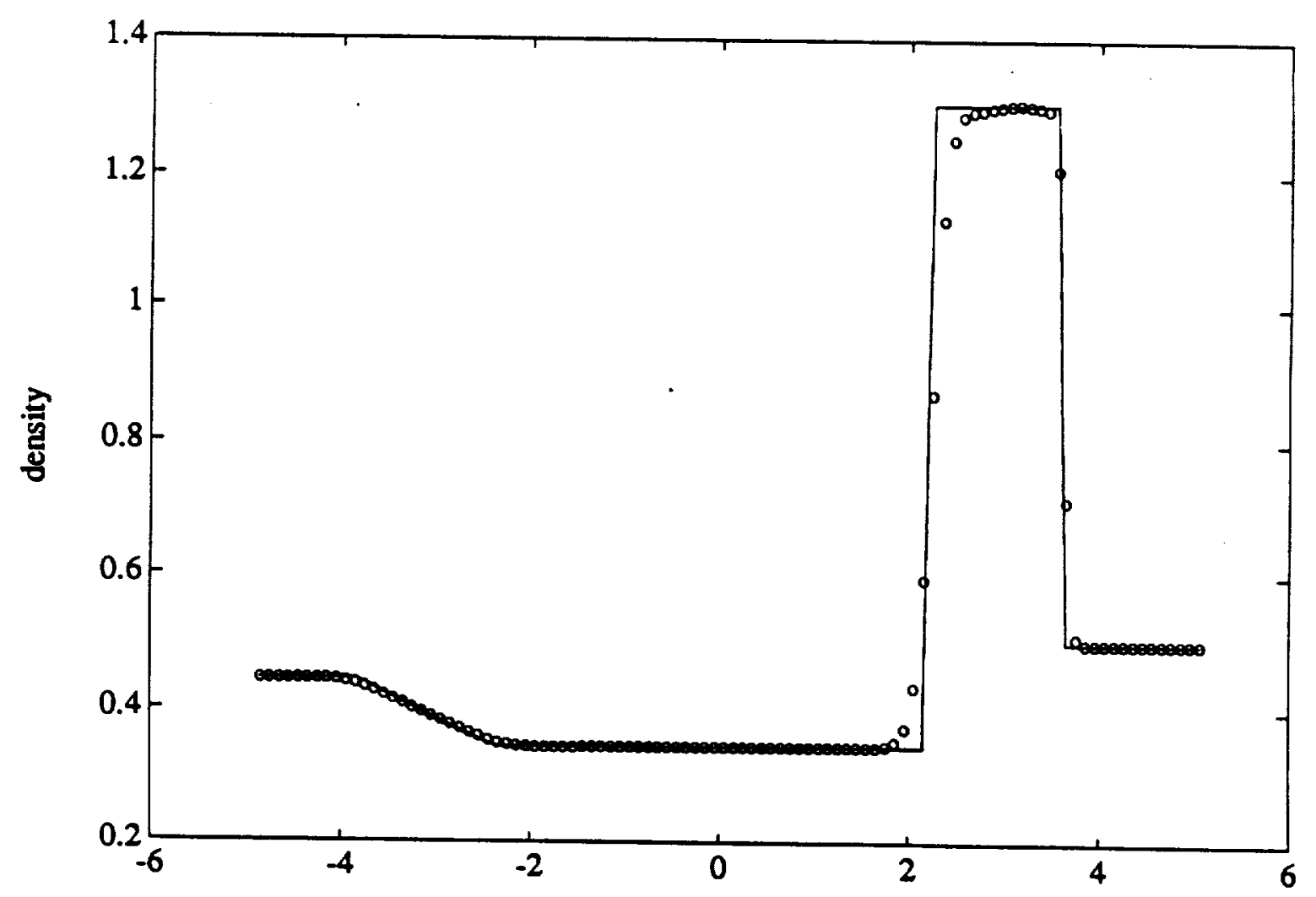

Figure 36.1: Lax problem, "4-th order" ENO without SM 


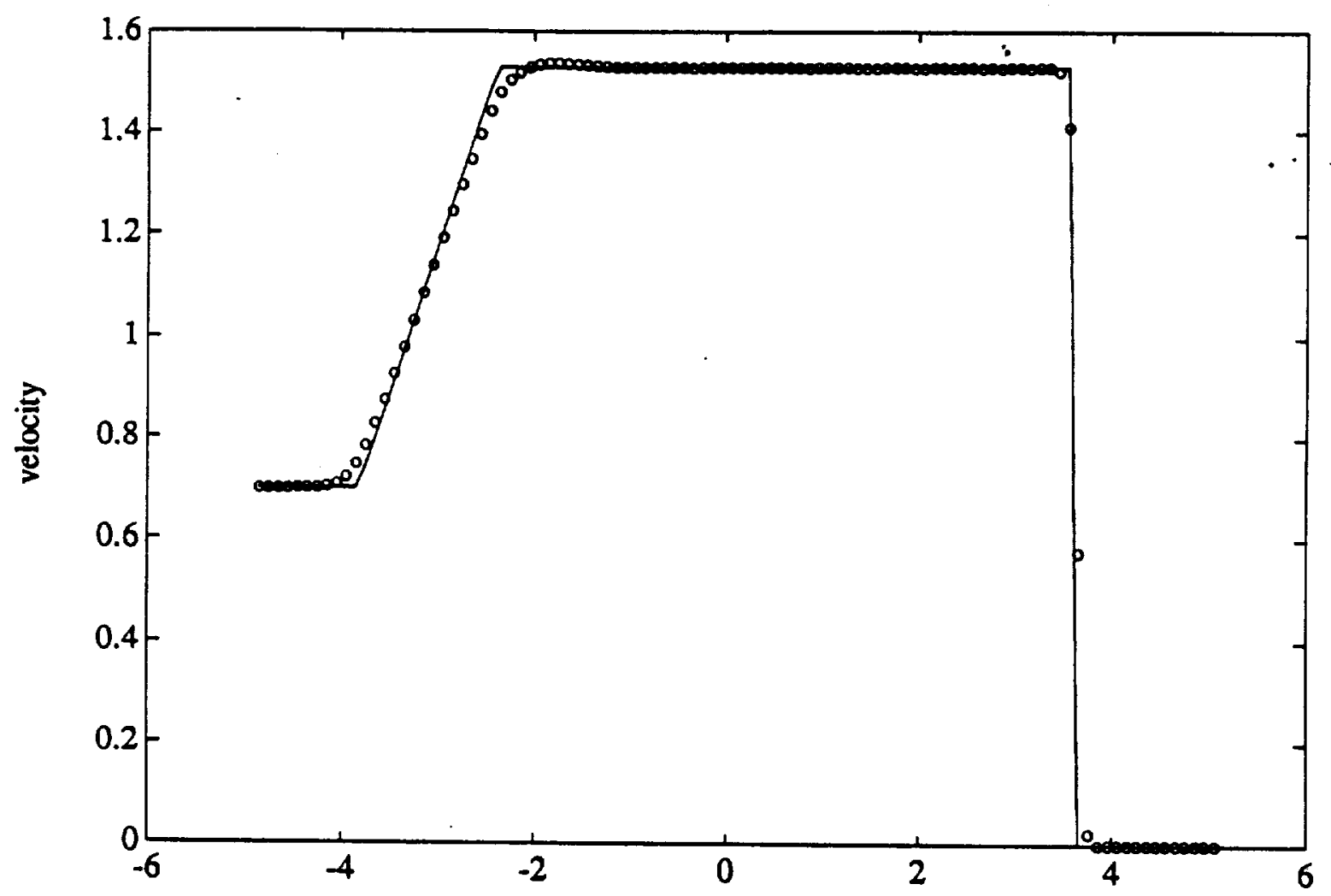

Figure 36.2: Lax problem, "4-th order" ENO without SM

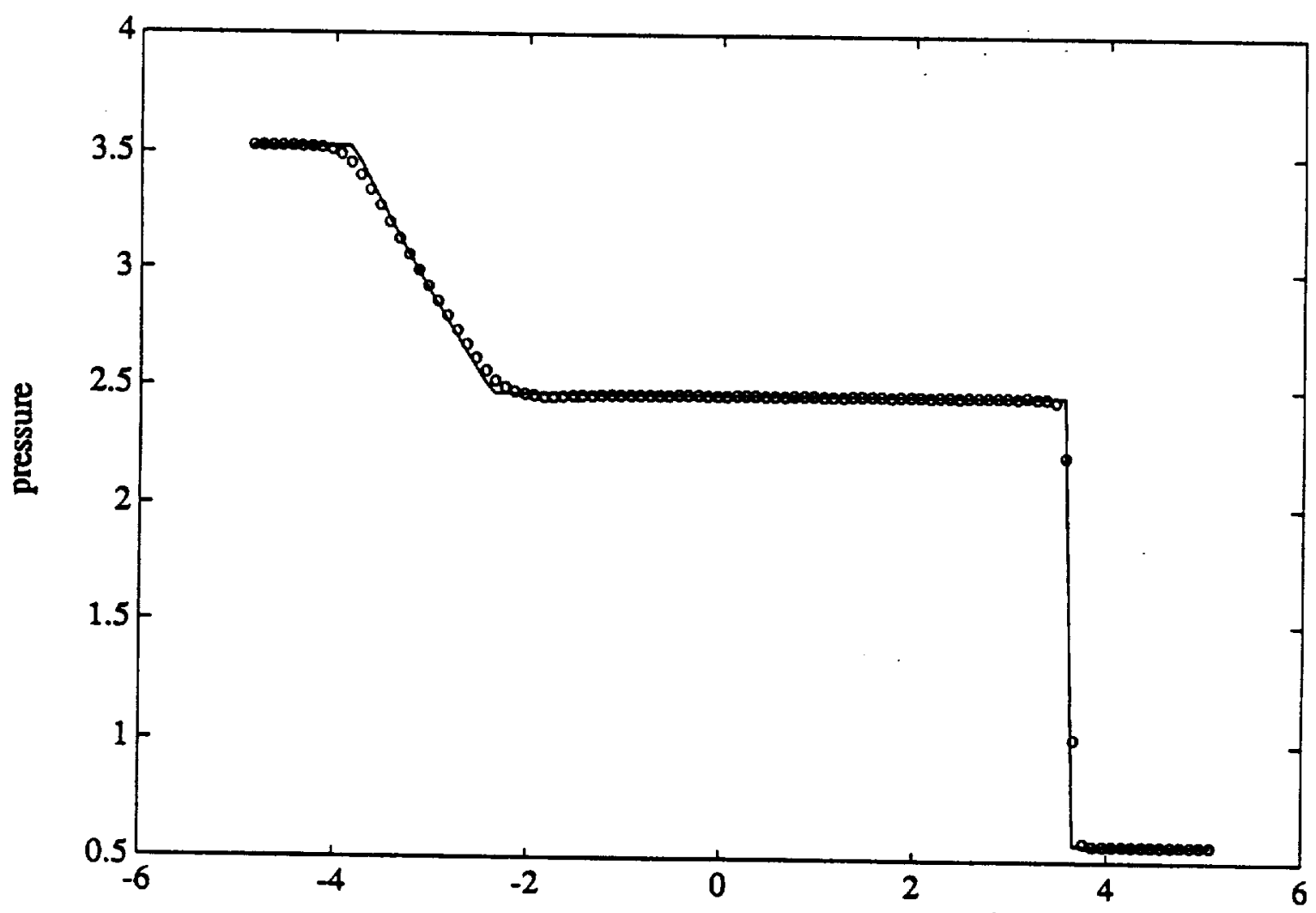

Figure 36.3: Lax problem, "4-th order" ENO without SM 


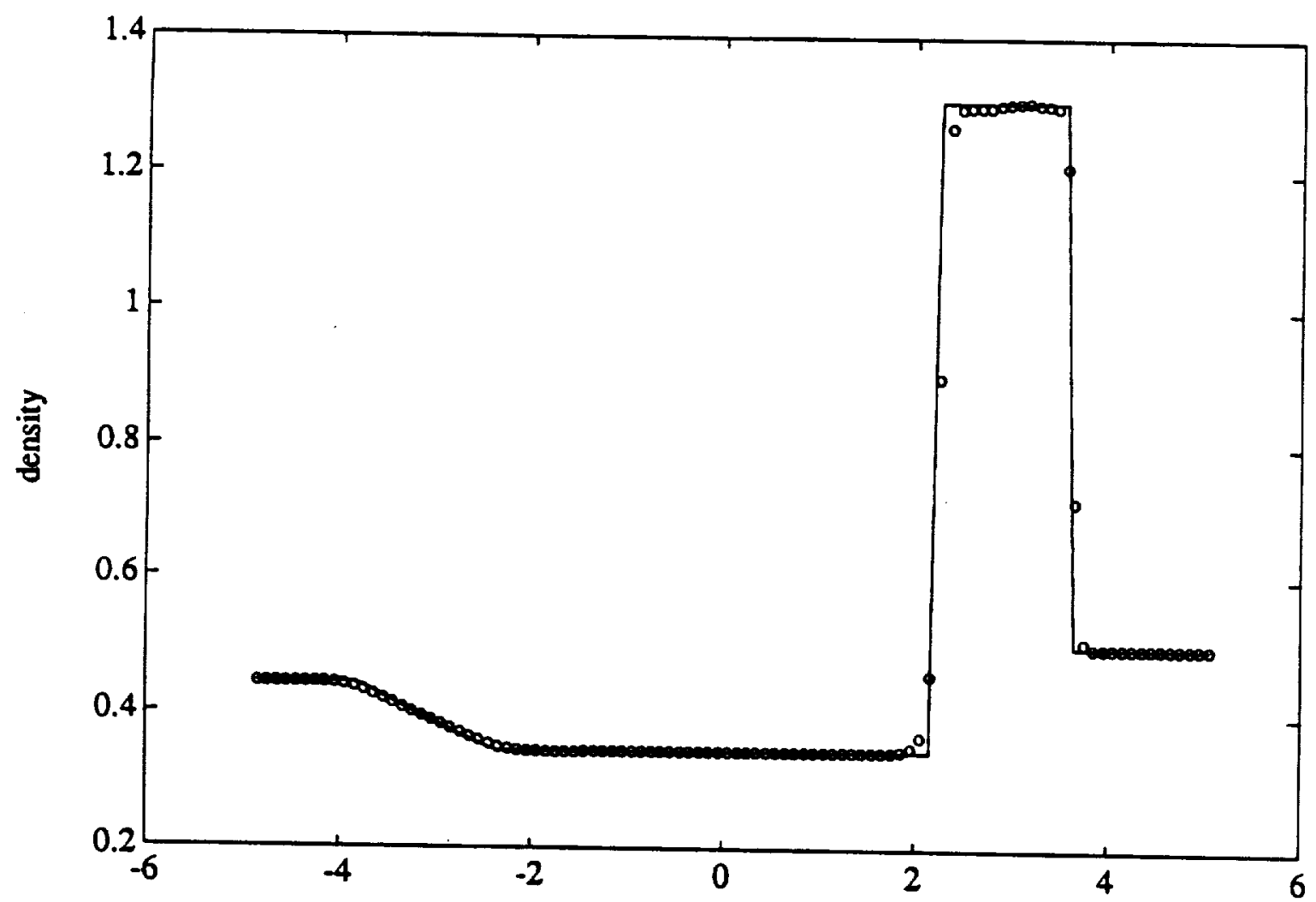

Figure 37.1: Lax problem, "4-th order" ENO with SM

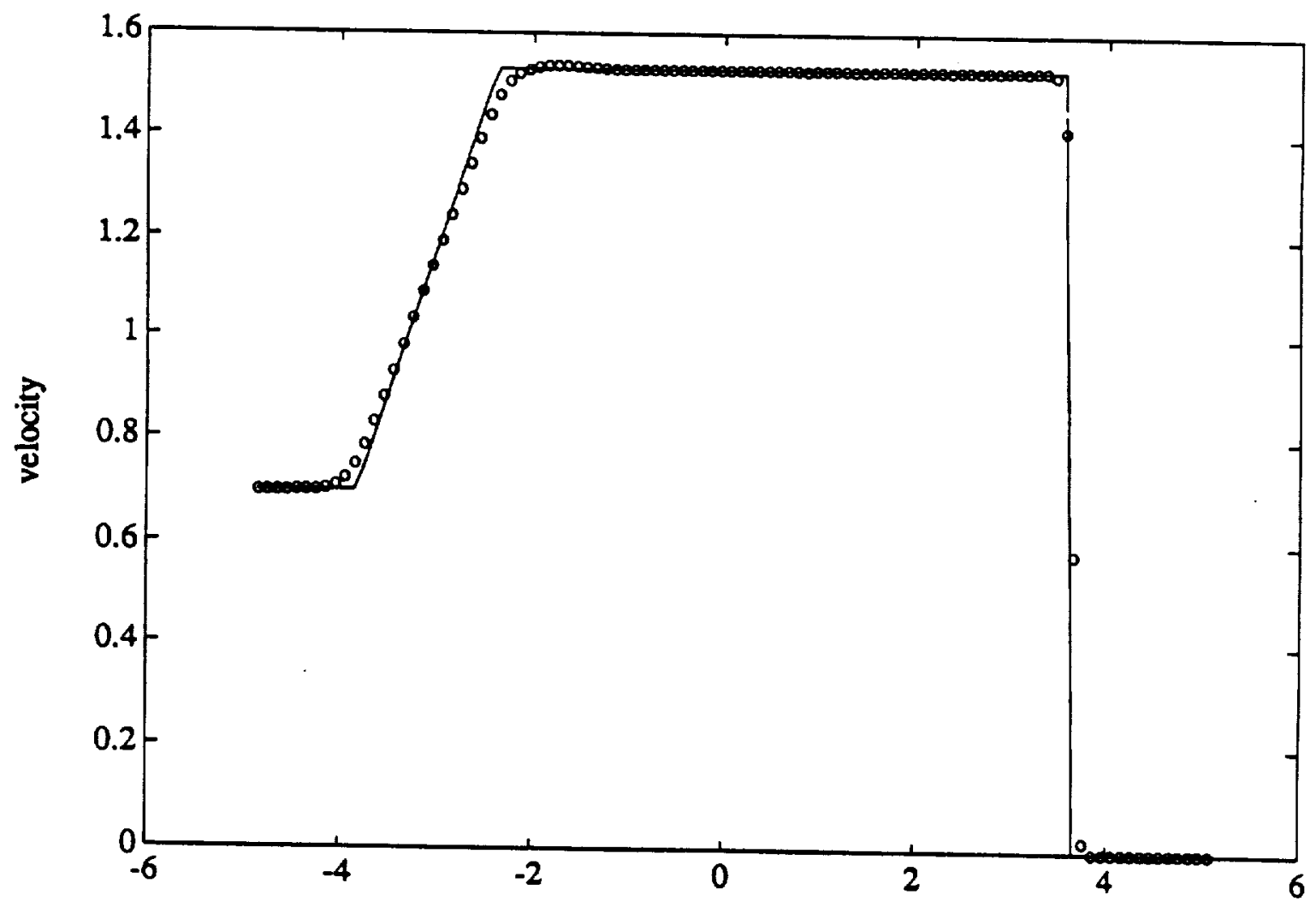

Figure 37.2: Lax problem, "4-th order" ENO with SM 


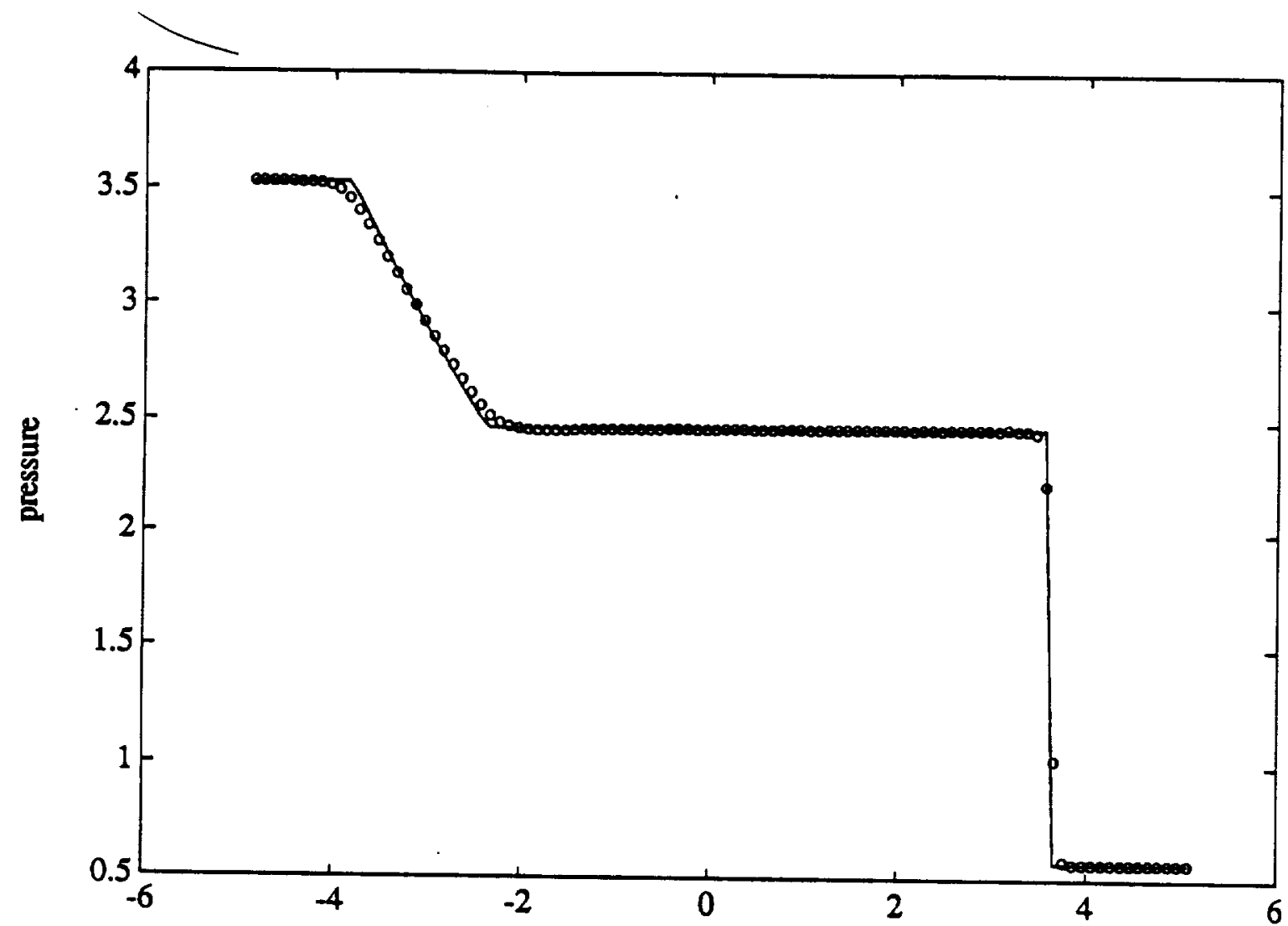

Figure 37.3: Lax problem, "4-th order" ENO with SM

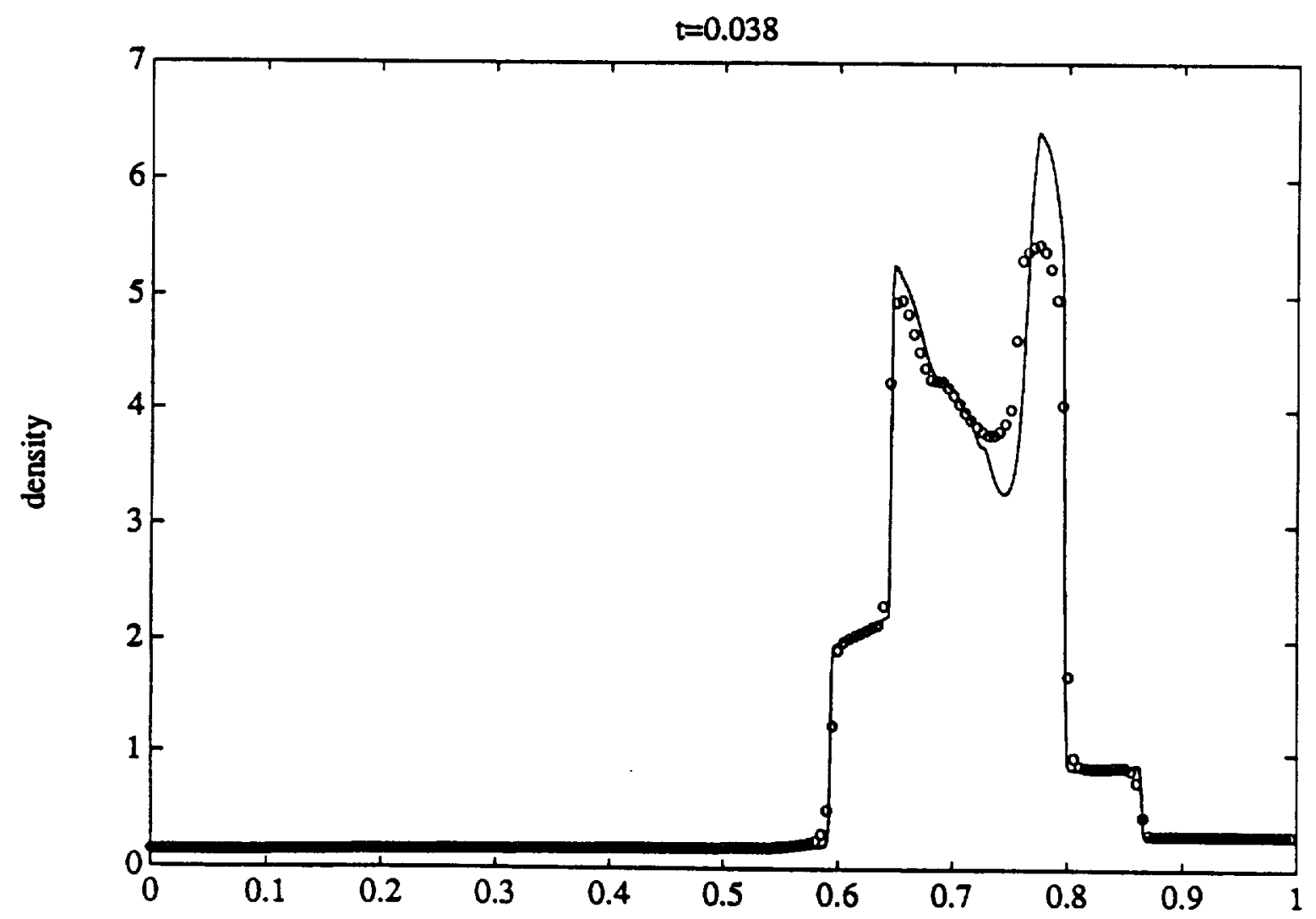

Figure 38.1: Blast wave, 2nd order ENO with SM 


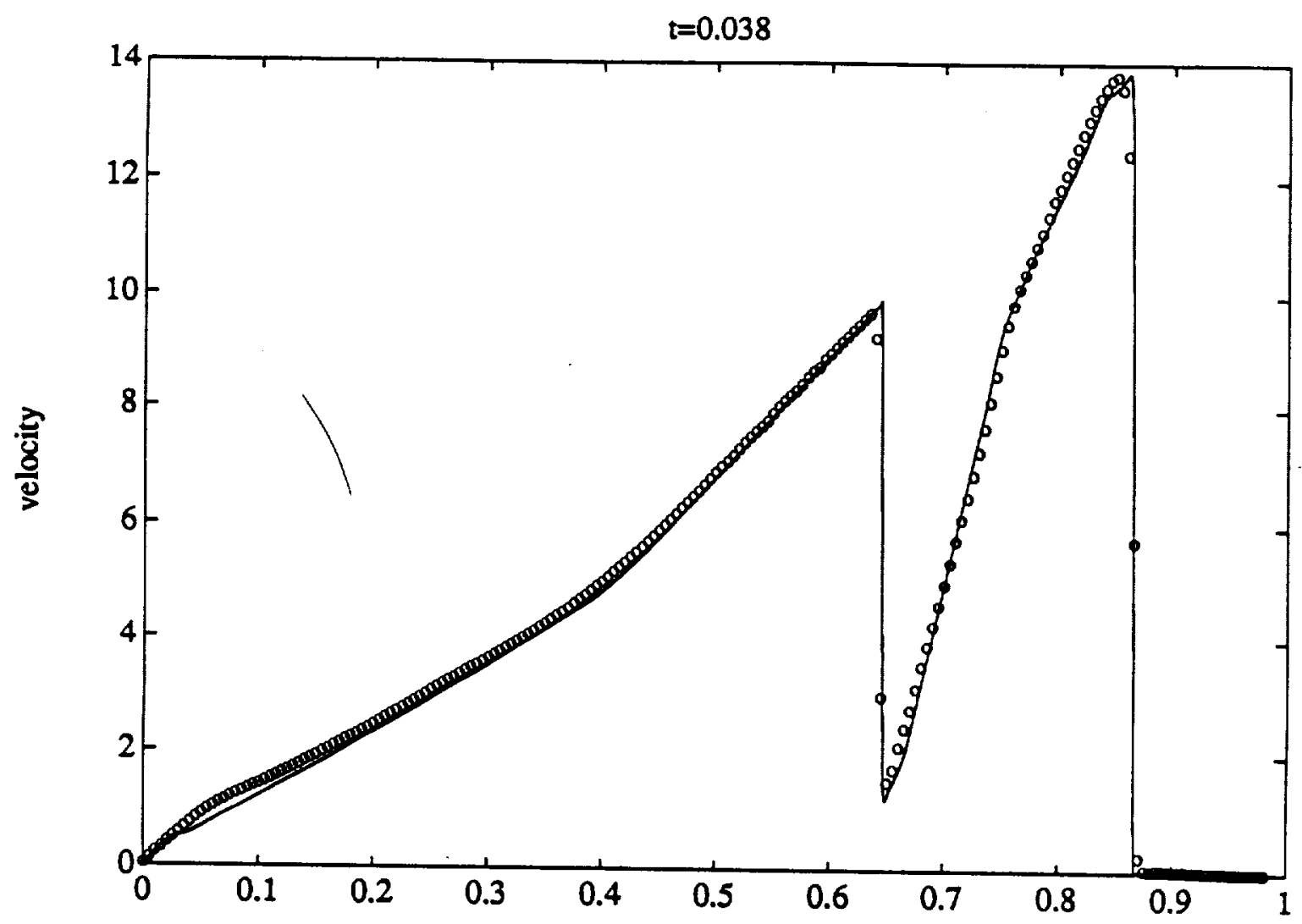

Figure 38.2: Blast wave, 2nd order ENO with SM

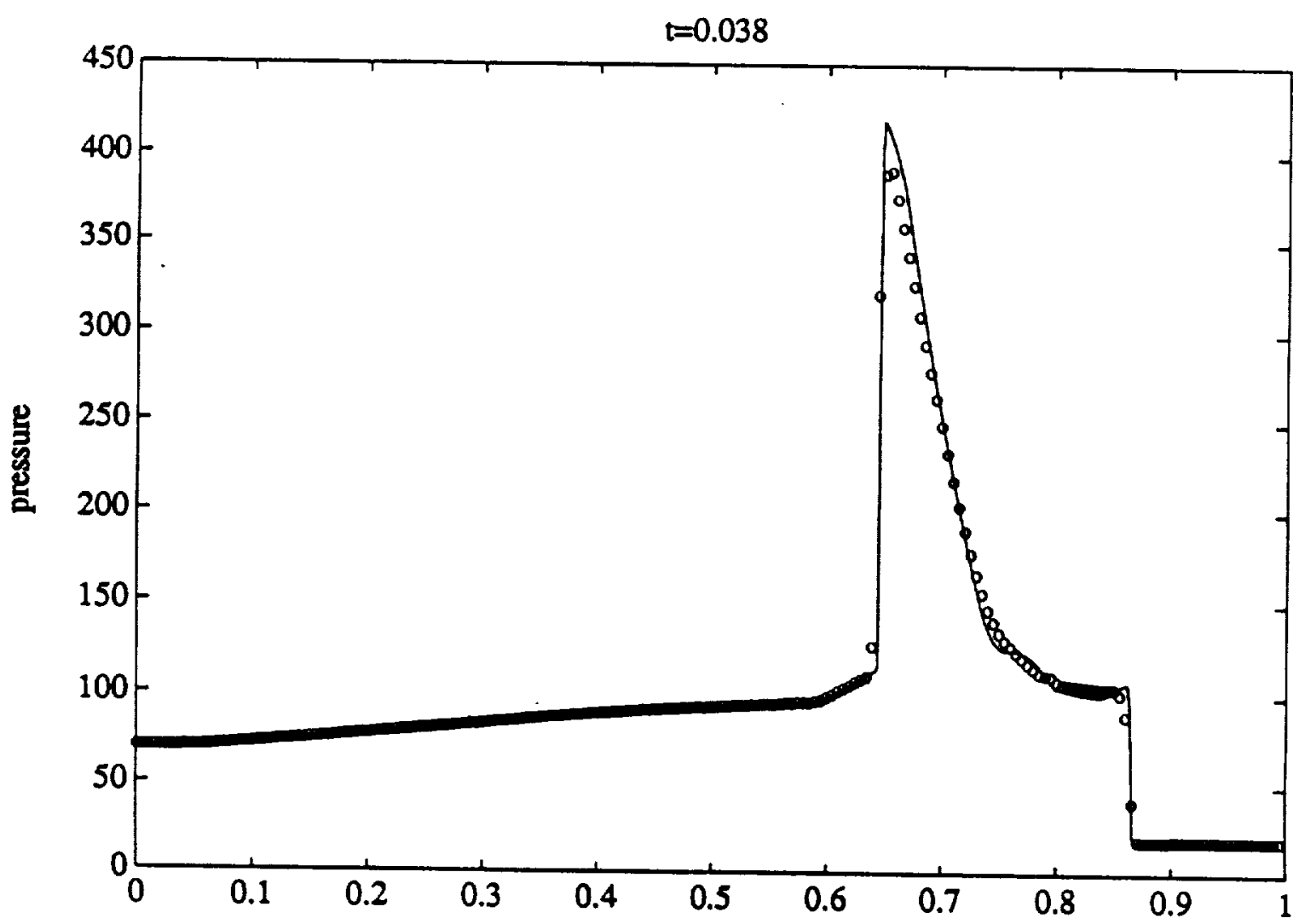

Figure 38.3: Blast wave, 2nd order ENO with SM 


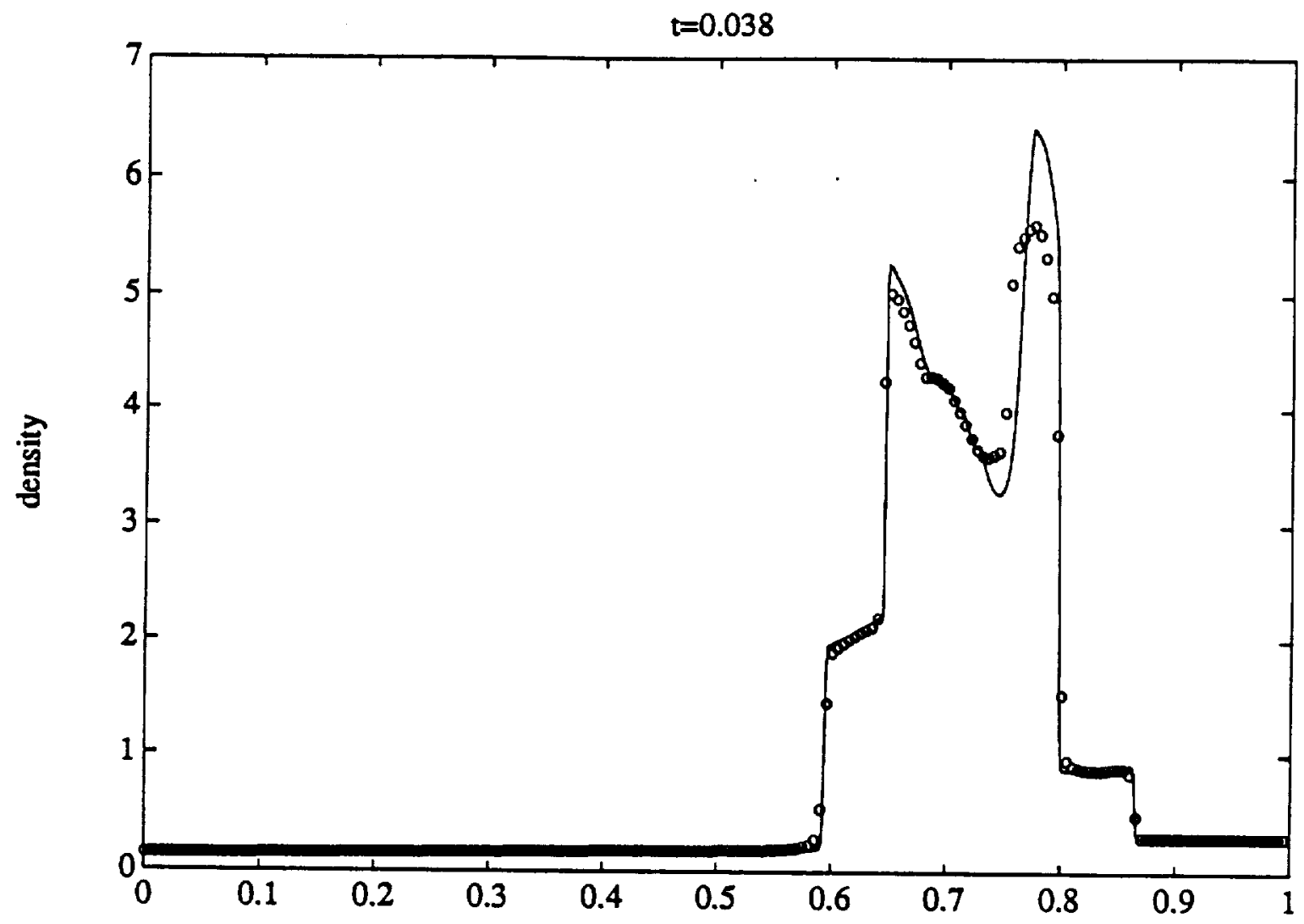

Figure 39.1: Blast wave, 4-th order ENO with SM

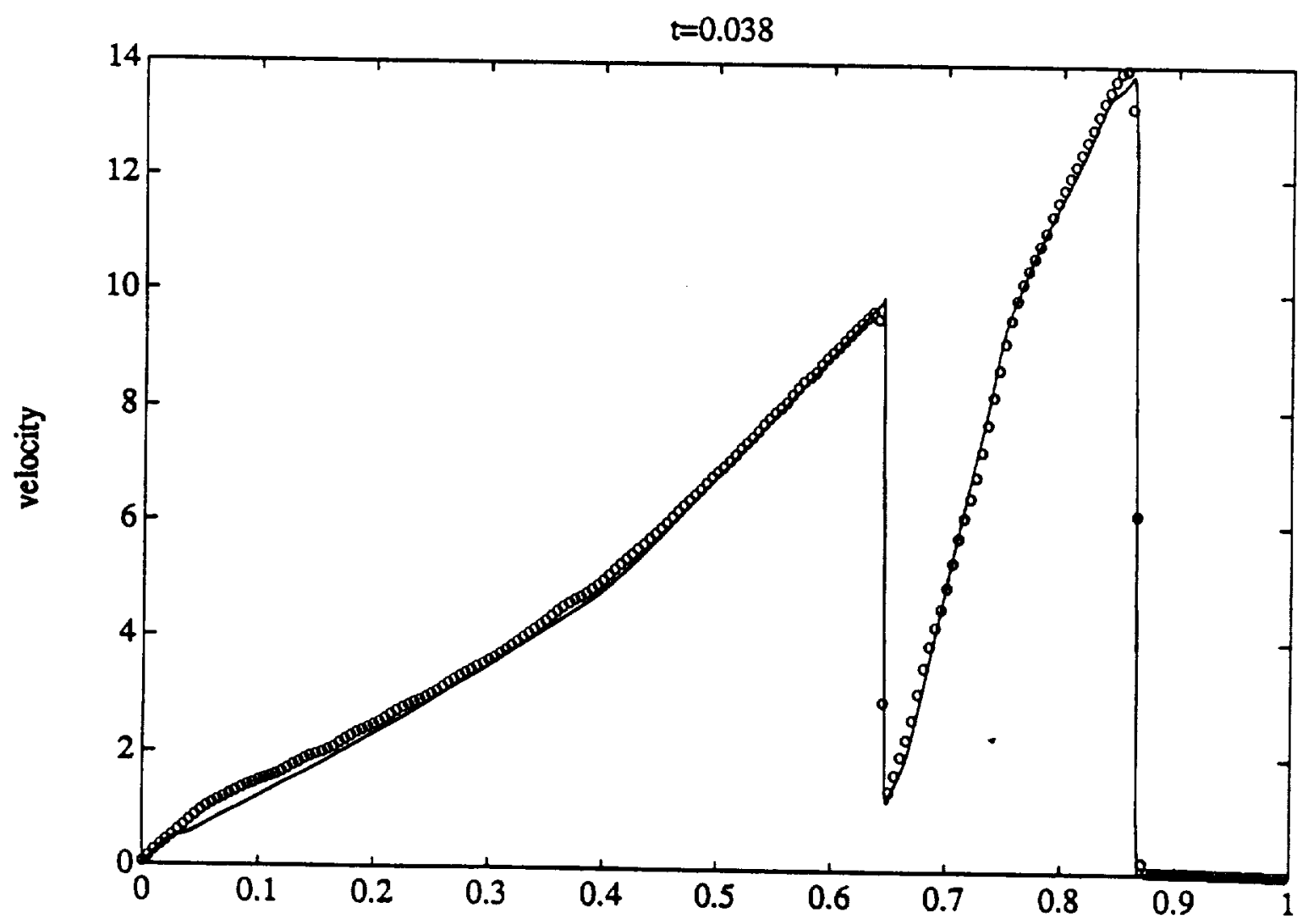

Figure 39.2: Blast wave, 4th order ENO with SM 


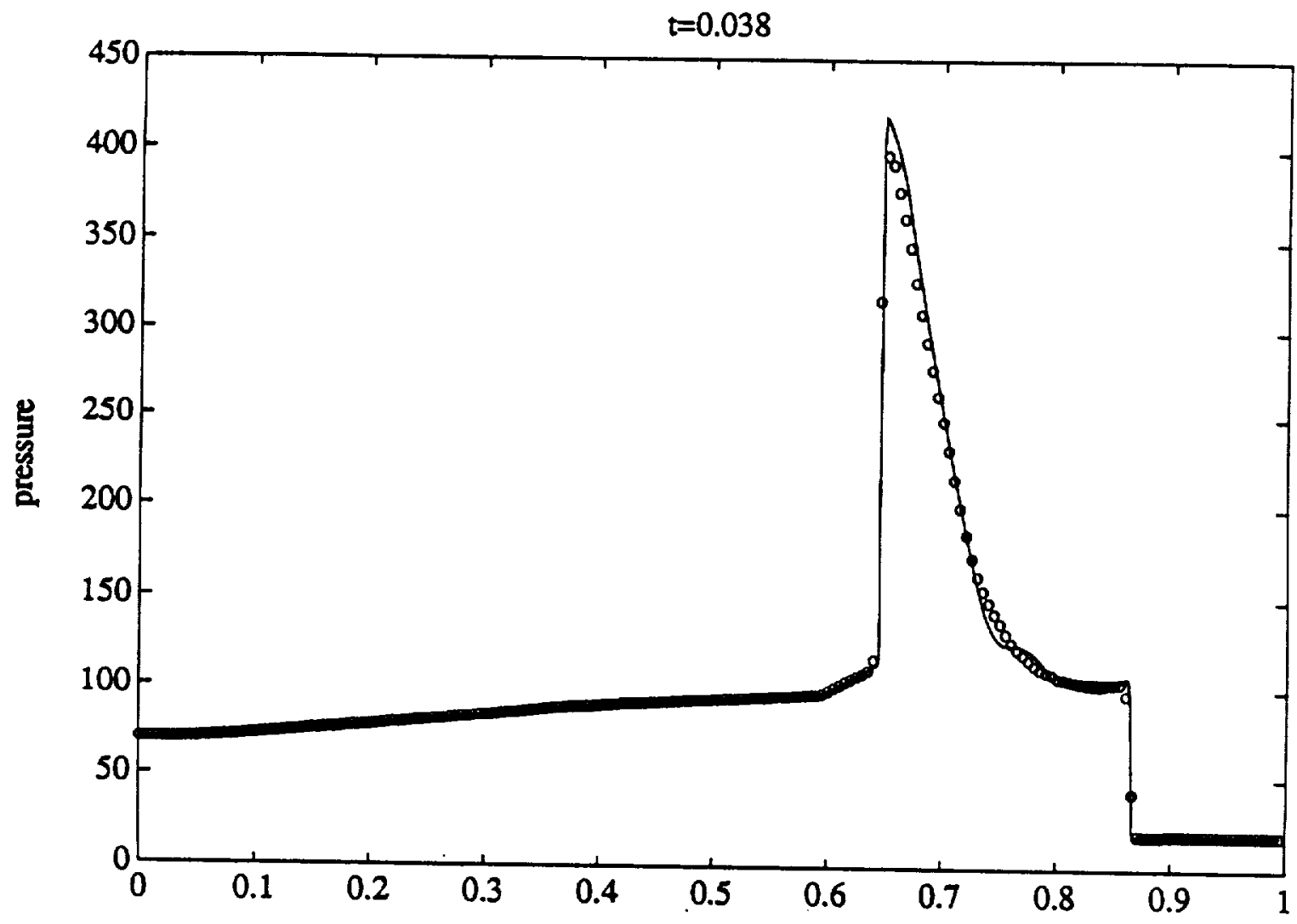

Figure 39.3: Blast wave, 4th order ENO with SM

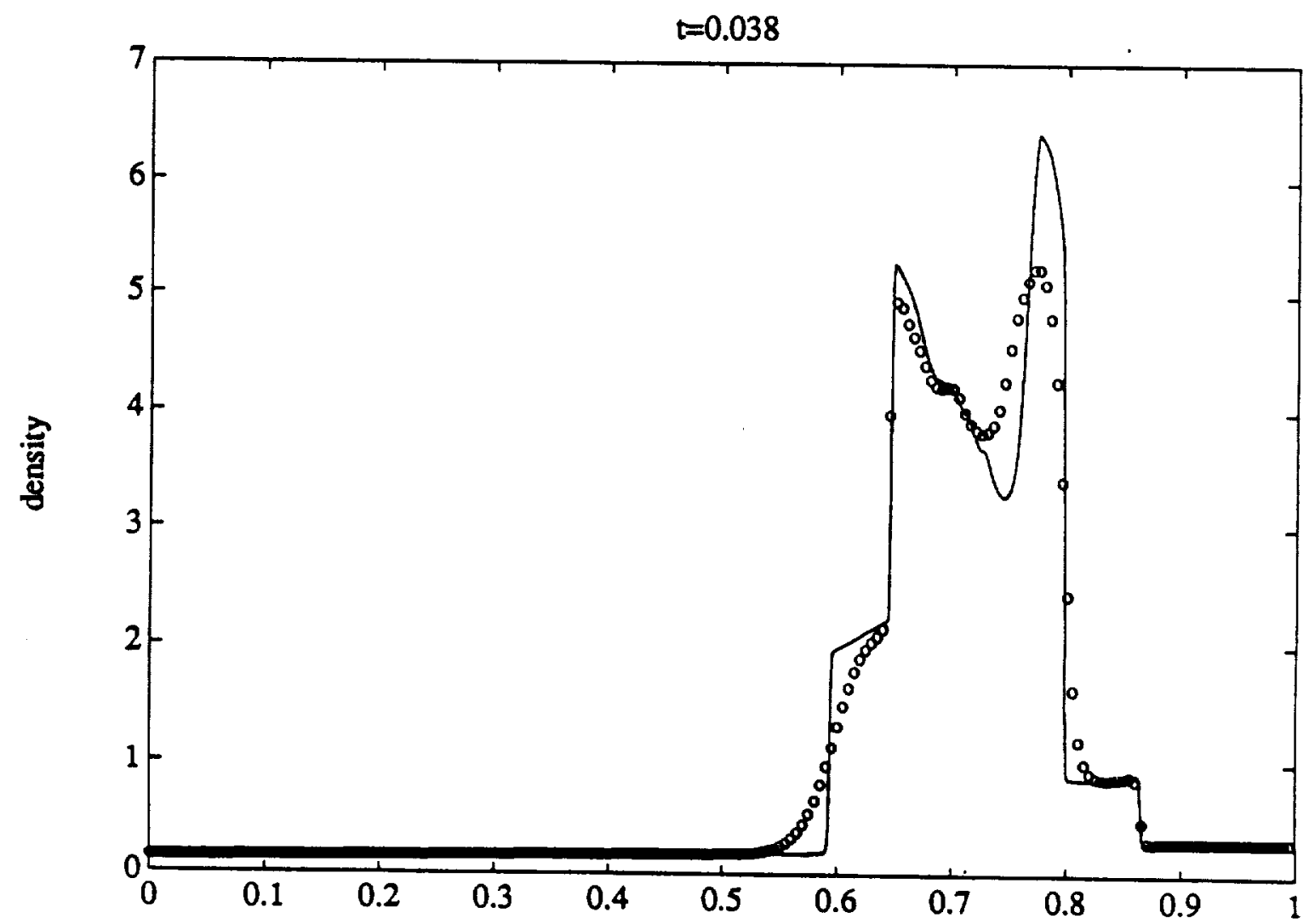

Figure 40.1: Blast wave, 4-th order ENO without SM 


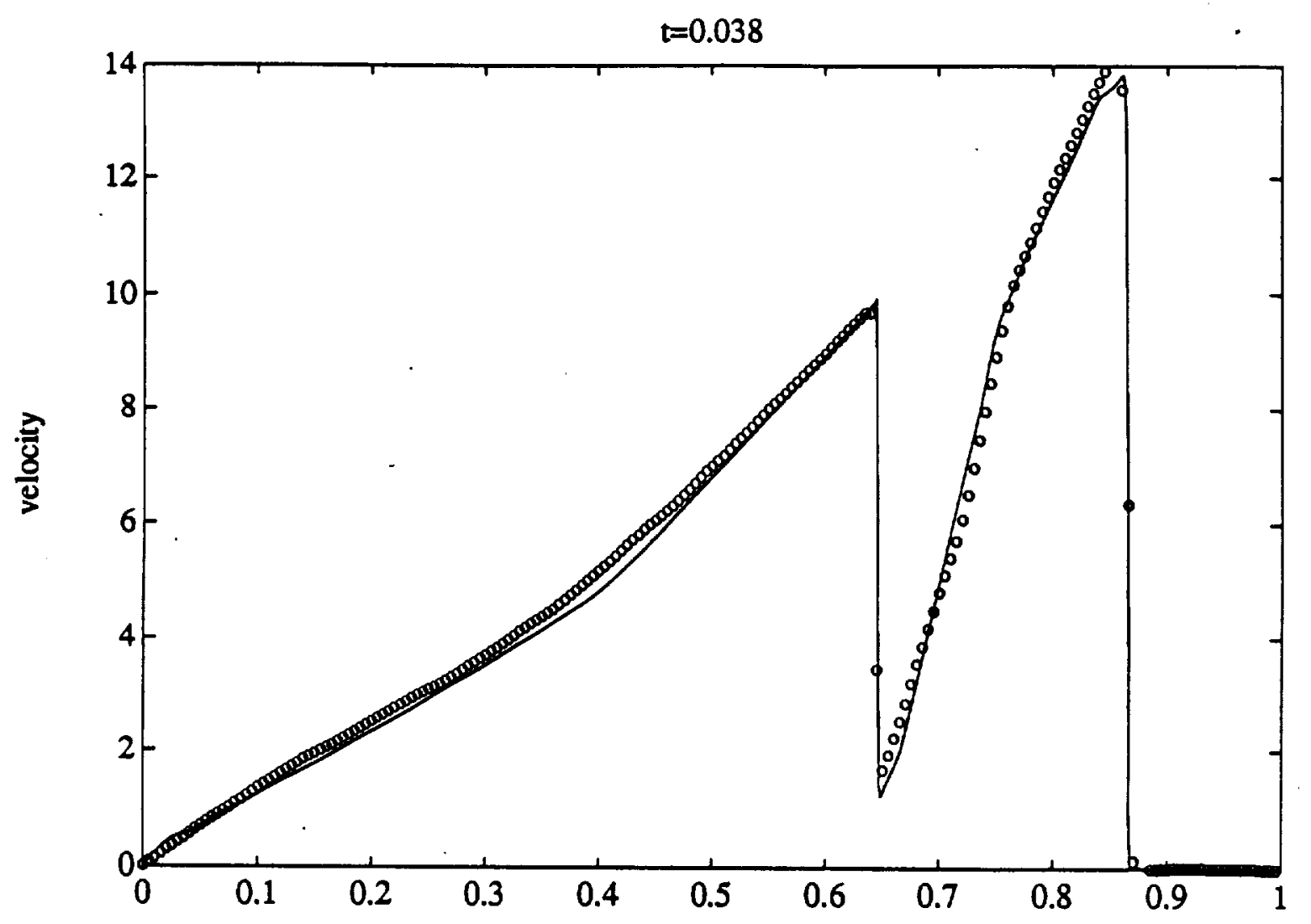

Figure 40.2: Blast wave, 4-th order ENO without SM

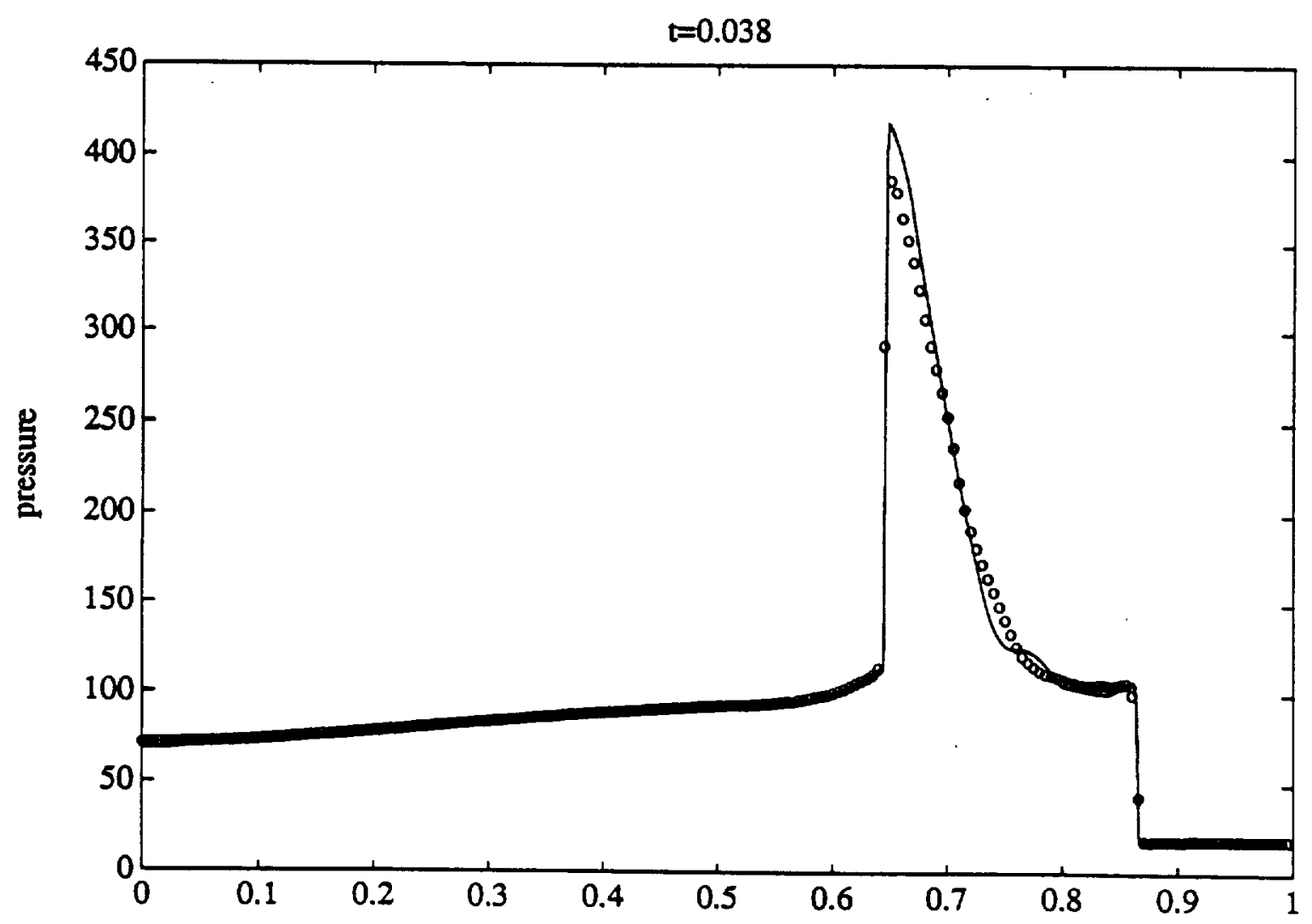

Figure 40.3: Blast wave, 4-th order ENO without SM 


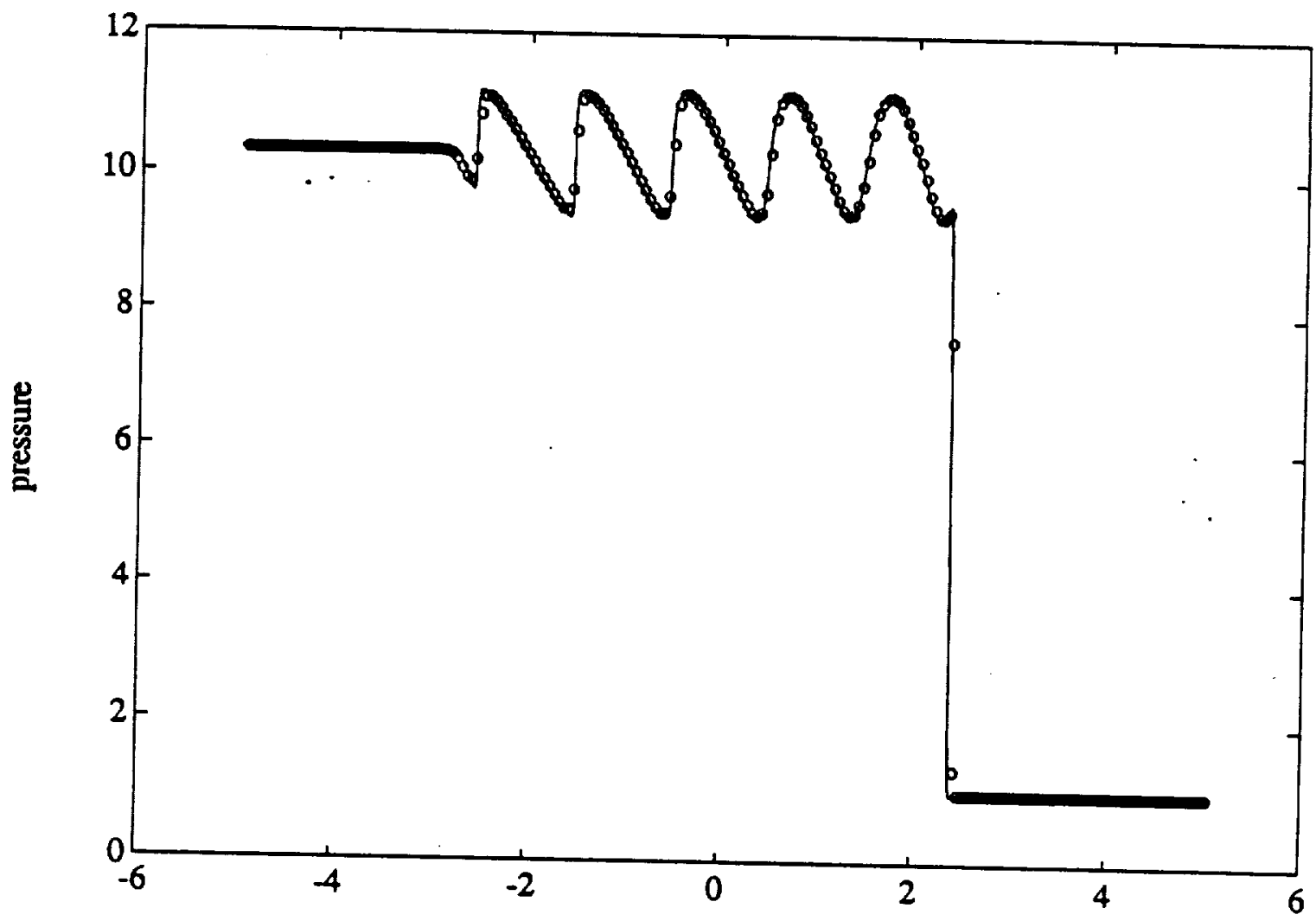

Figure 41.3: shock/turbulence, 4-th order ENO with SM

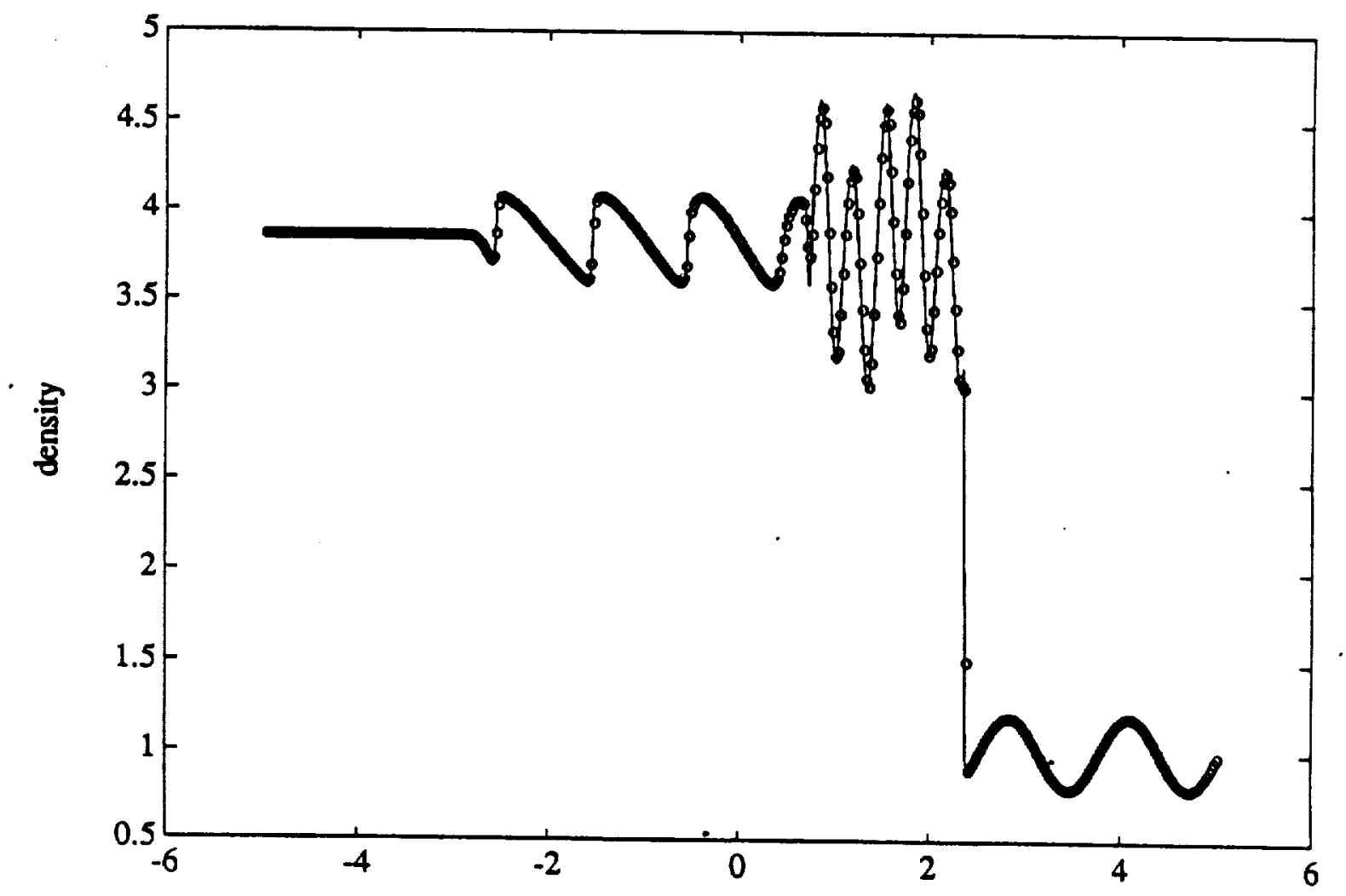

Figure 42.1: shock/turbulence, 4-th order ENO with SM 


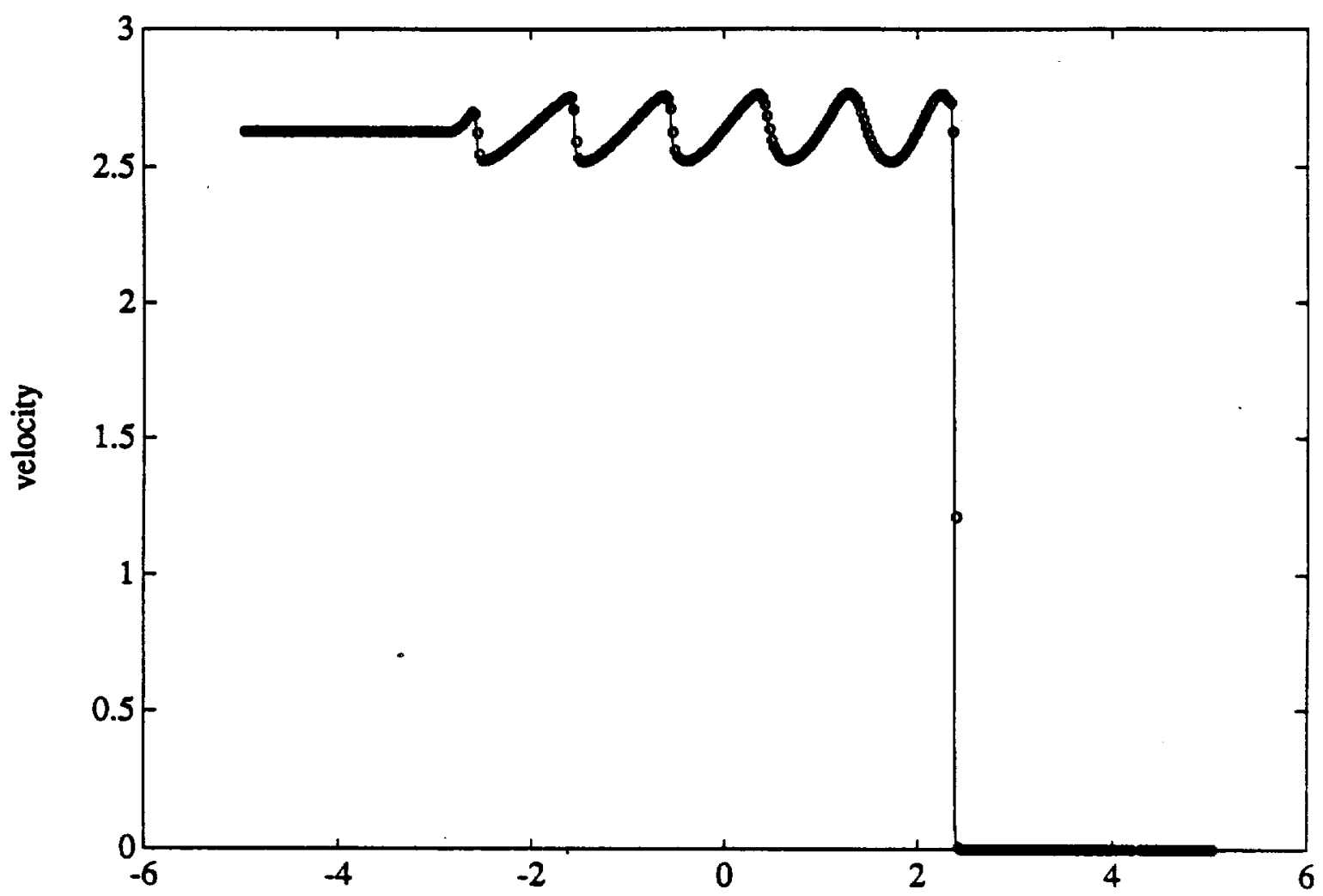

Figure 42.2: shock/turbulence, 4-th order ENO with SM

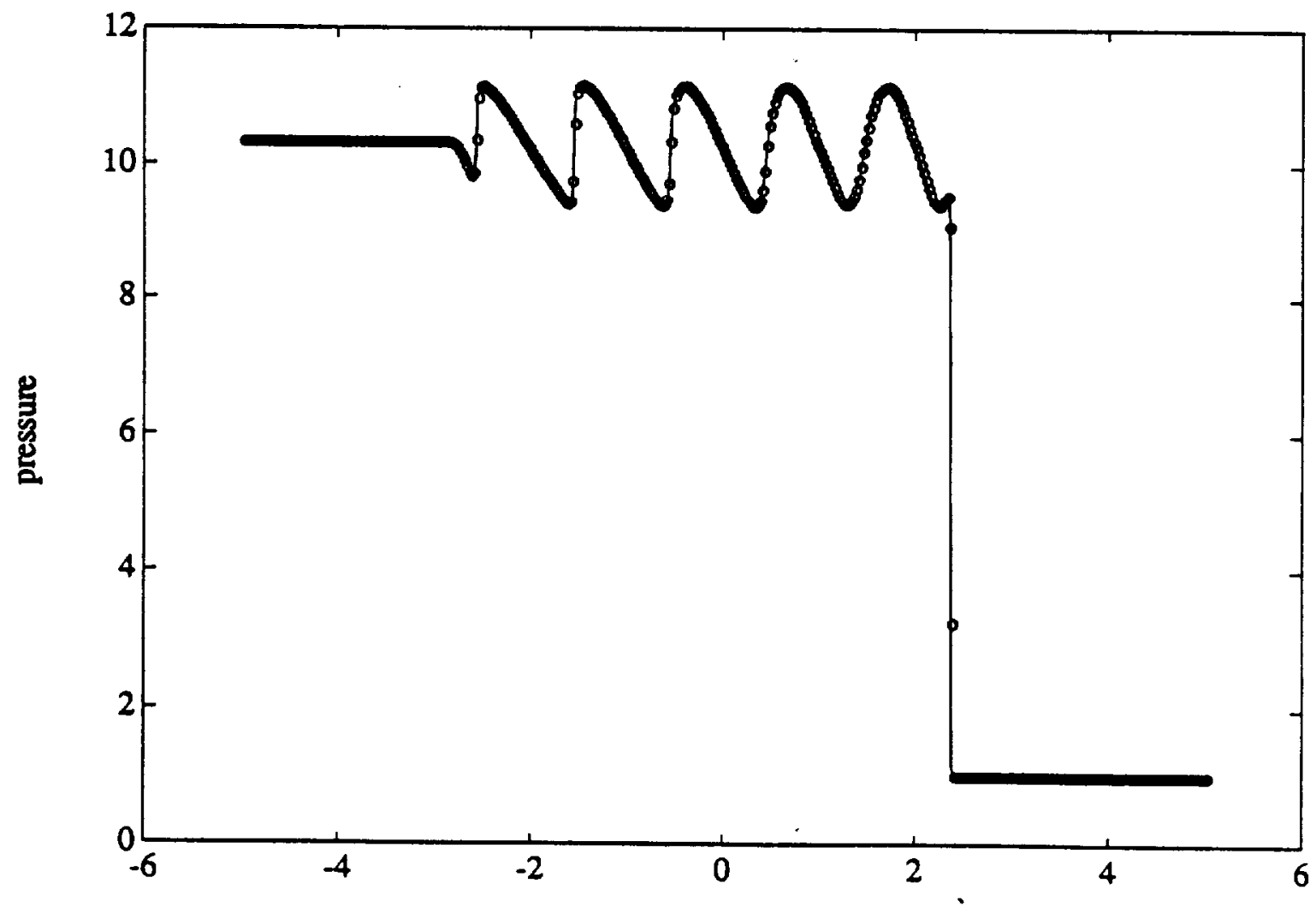

Figure 42.3: shock/turbulence, 4-th order ENO with SM 


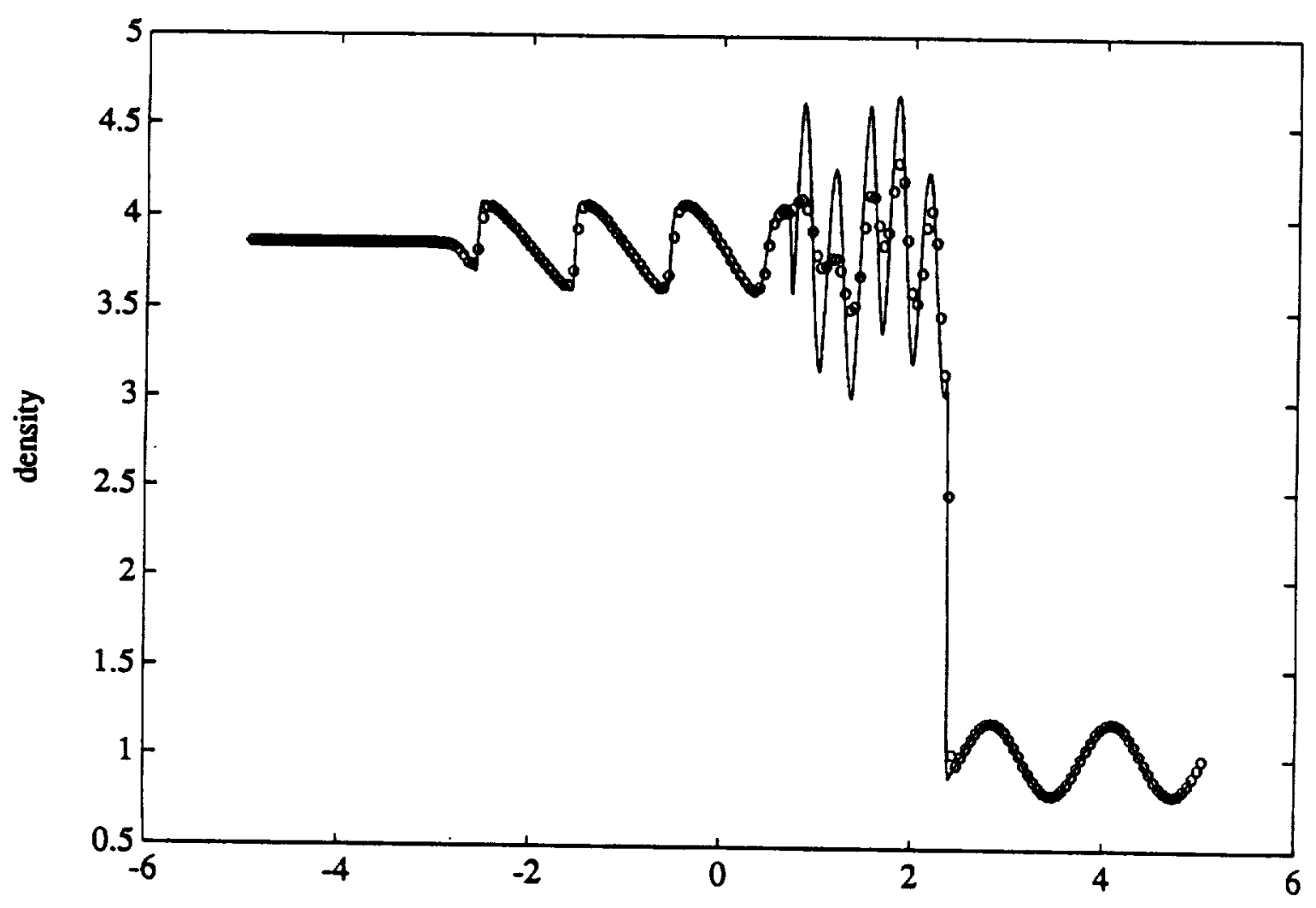

Figure 43.1: shock/turbulence, 4-th order ENO without SM

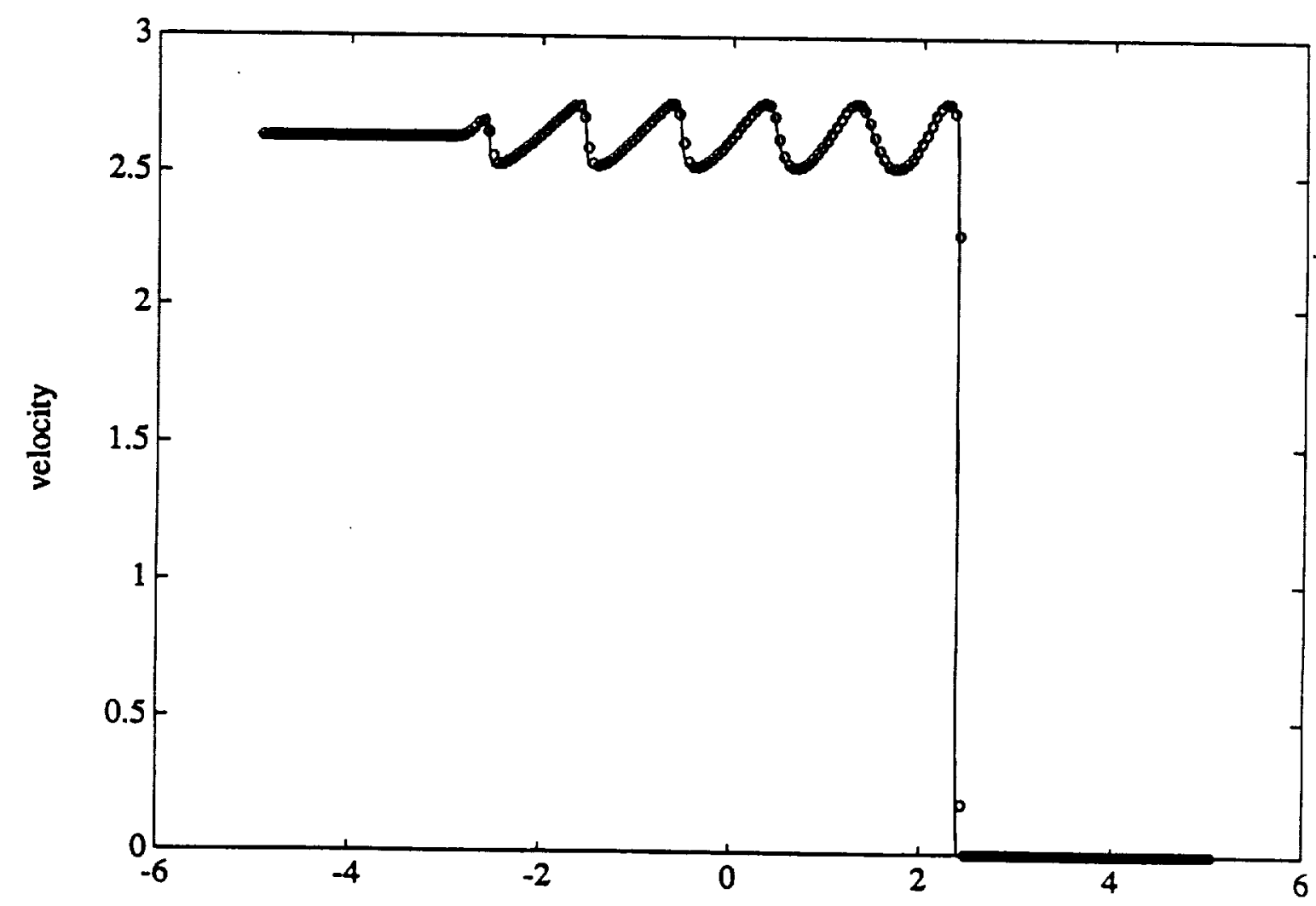

Figure 43.2: shock/turbulence, 4th order ENO without SM 


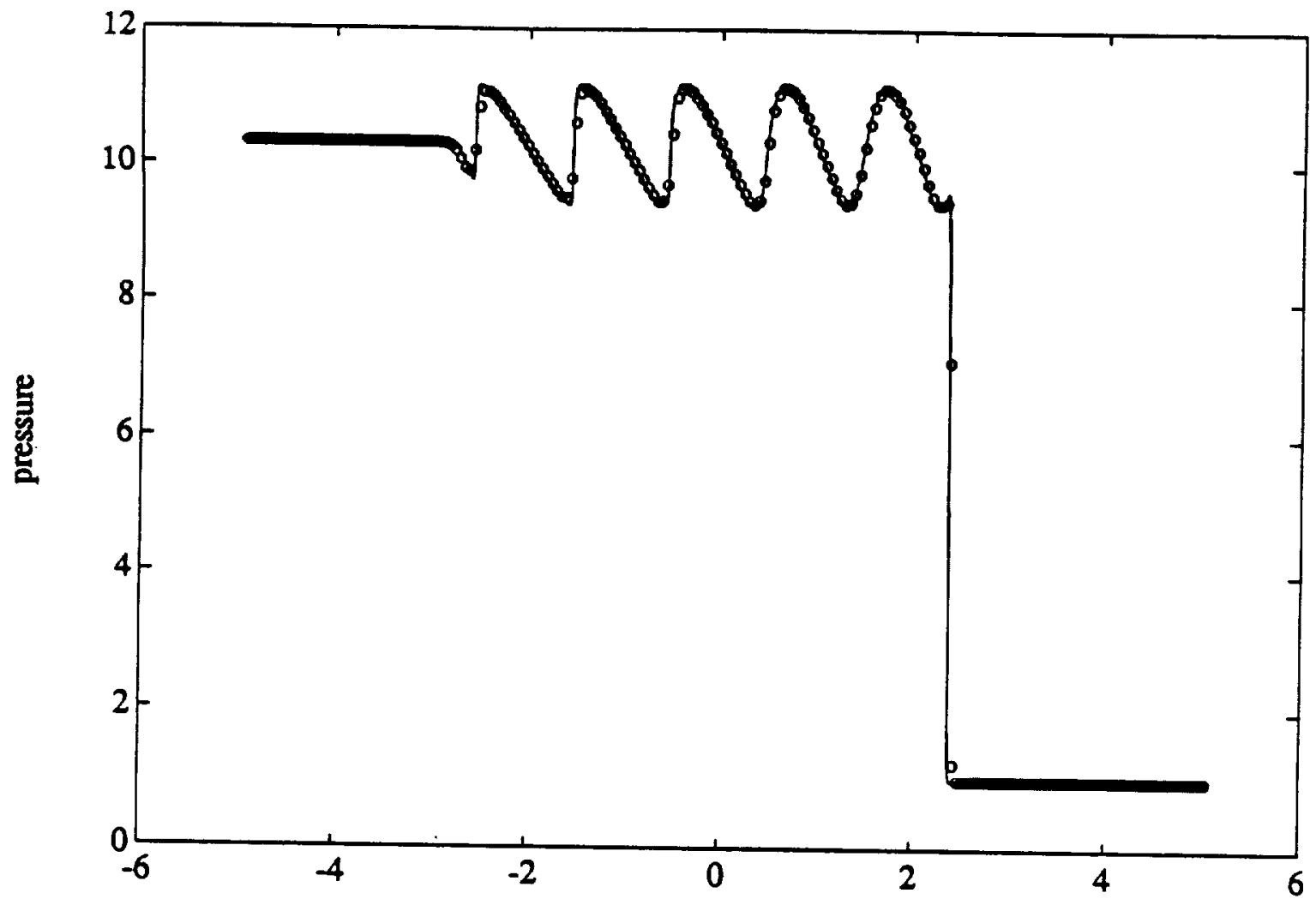

Figure 43.3: shock/turhulence. 4th ordar FNn withmen cMx

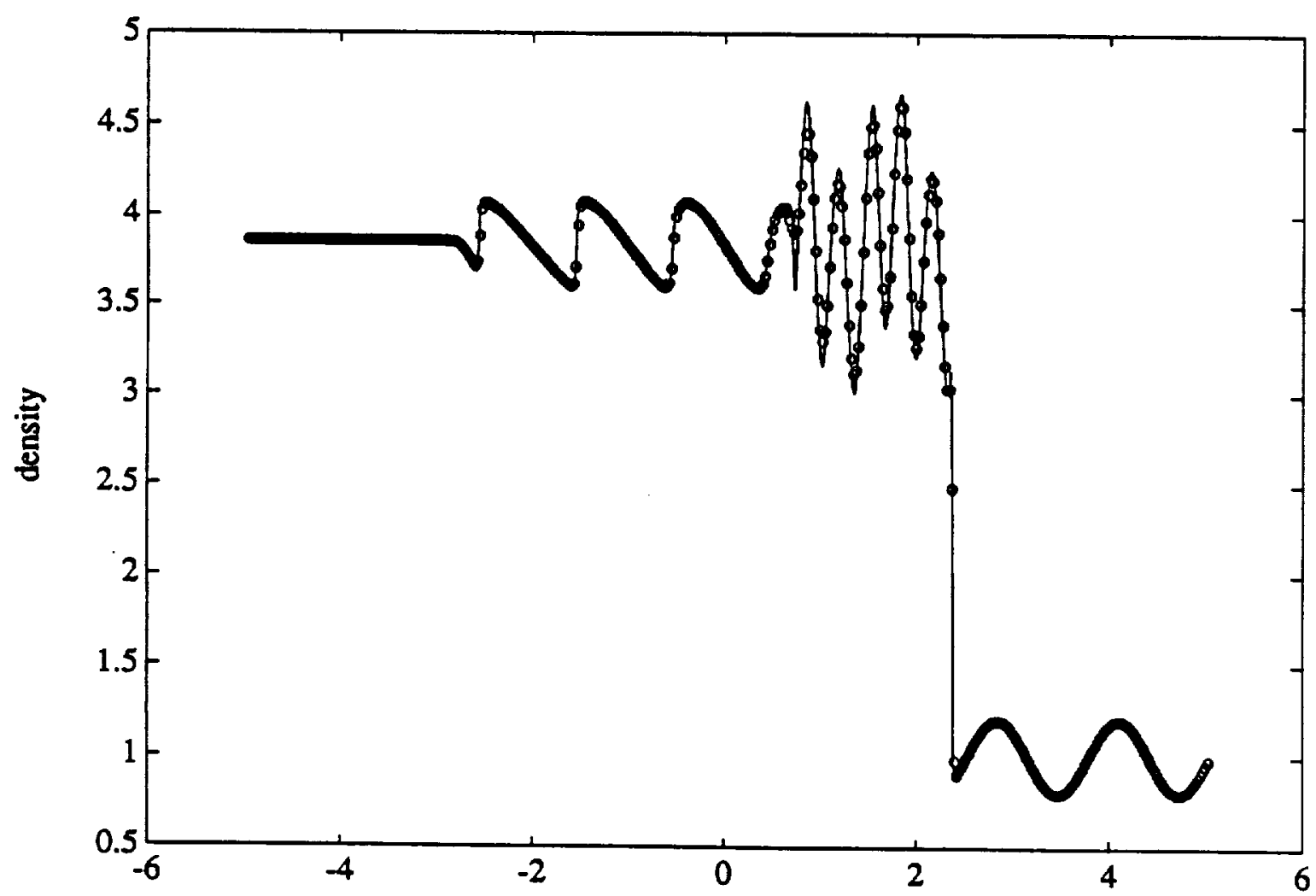

Figure 44.1: shock/turbulence, 4-th order ENO without SM 


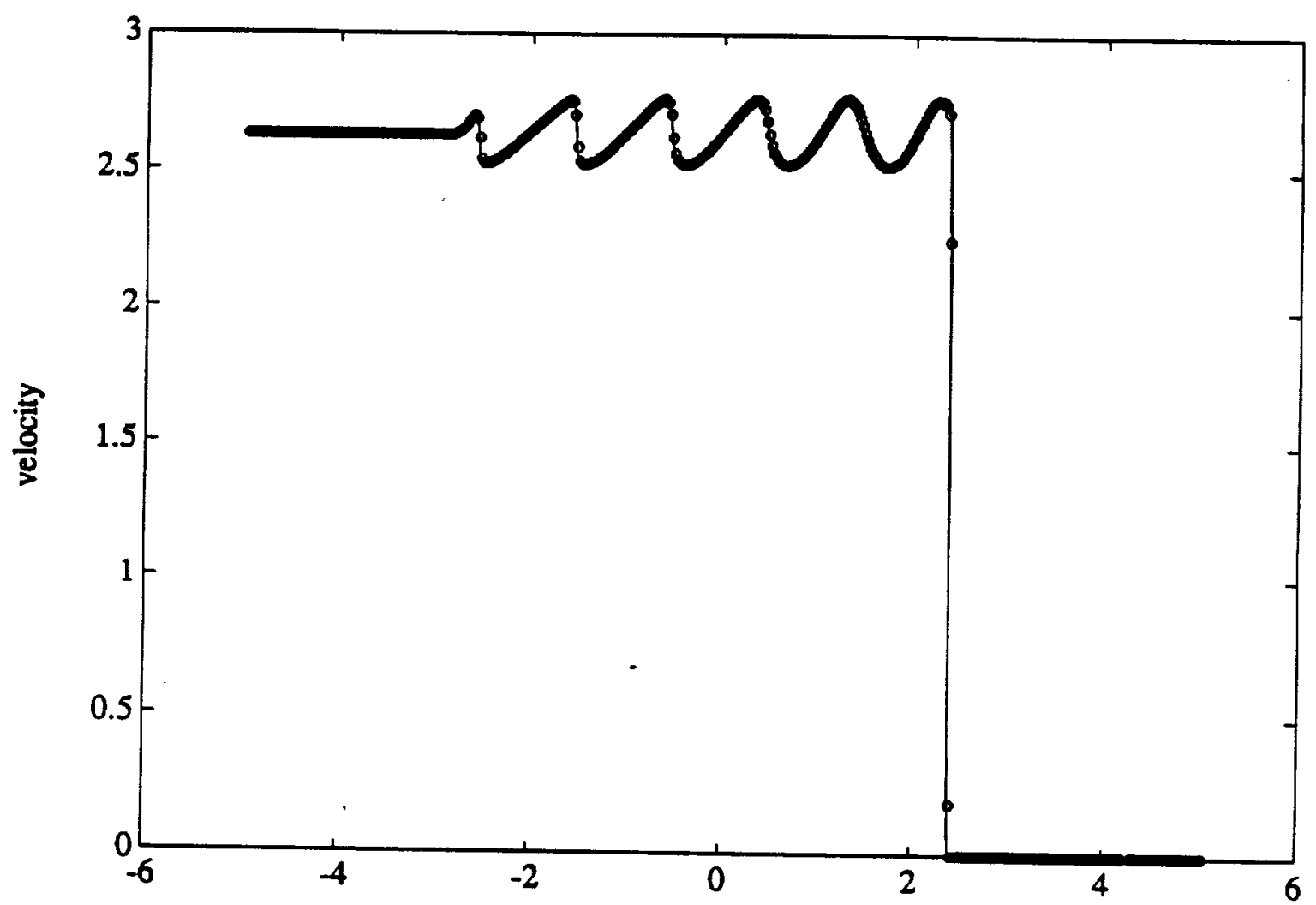

Figure 44.2: shock/turbulence, 4th order ENO without SM

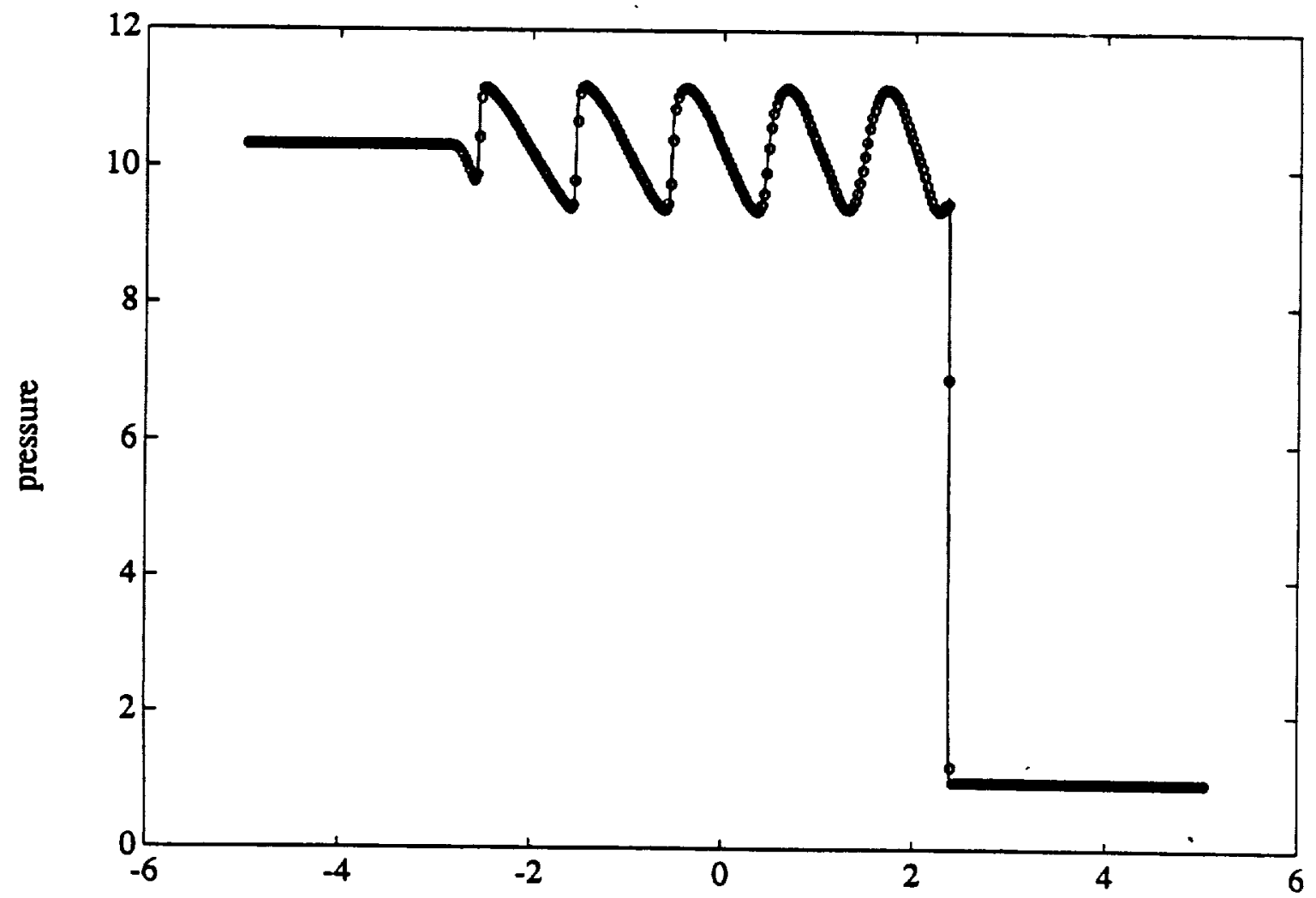

Figure 44.3: shock/turbulence, 4-th order ENO without SM 


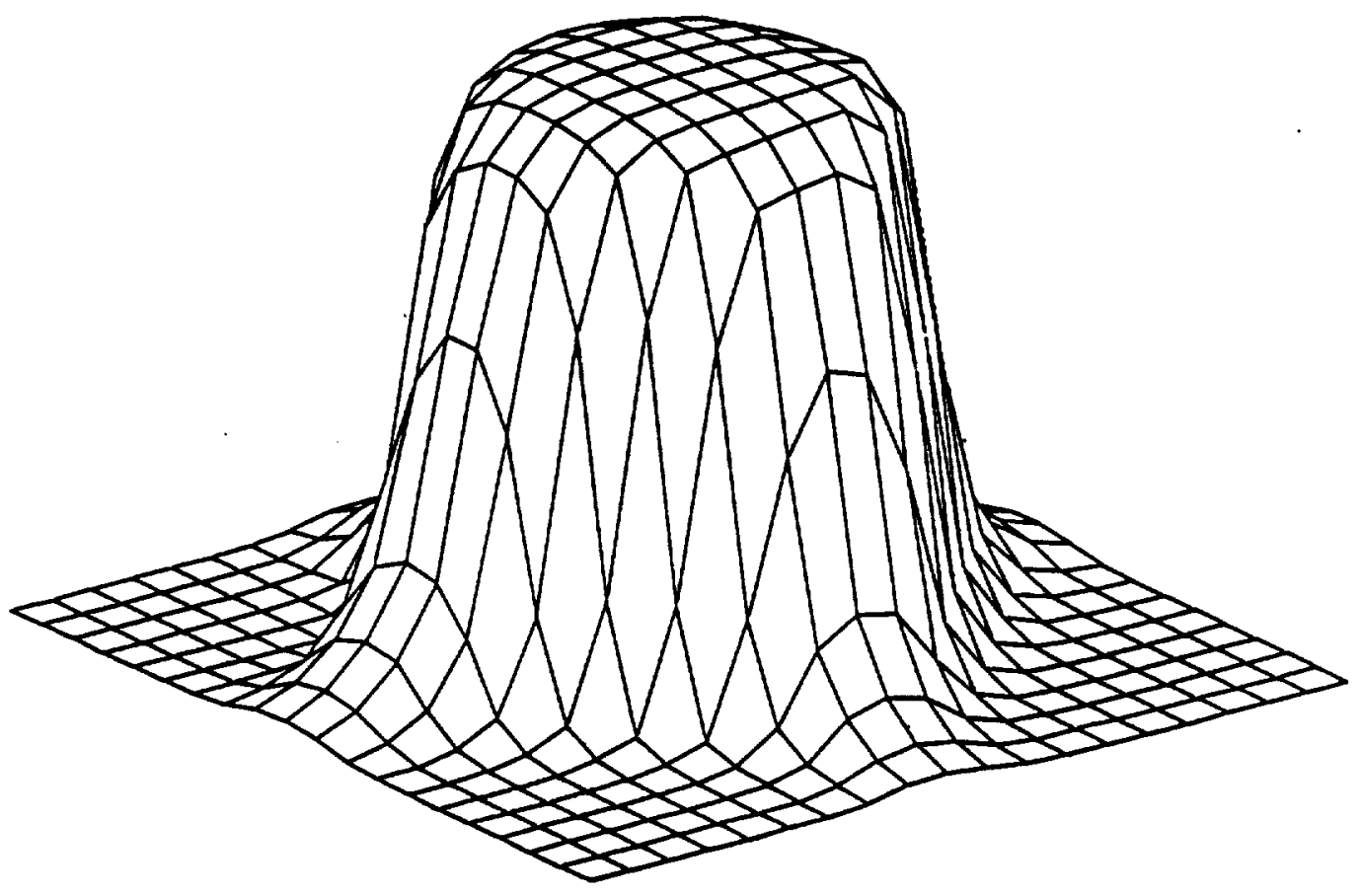

Figure 45: 2nd order ENO, with artificial compression

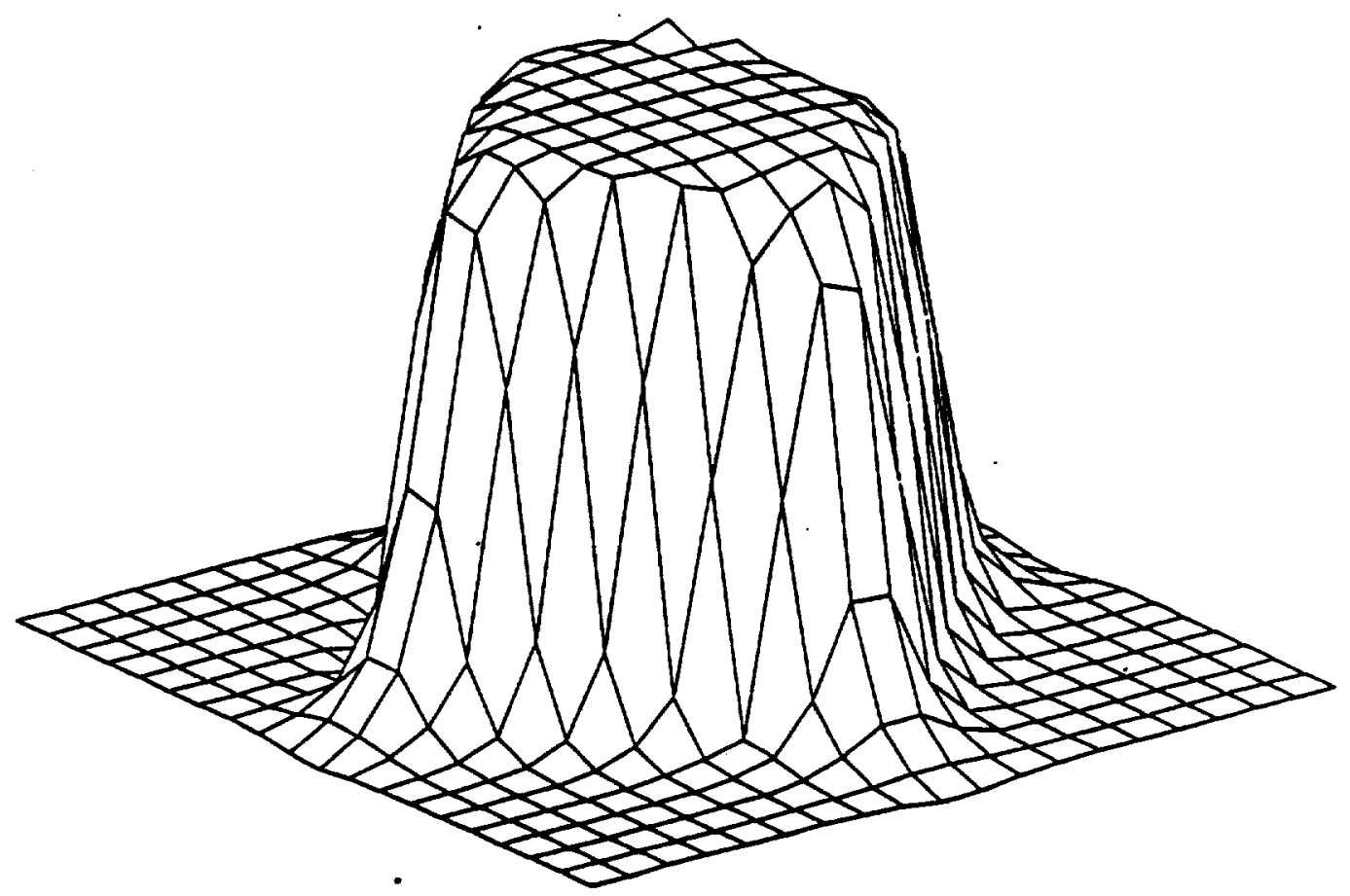

Figure 46: 3rd order ENO, with artificial compression 


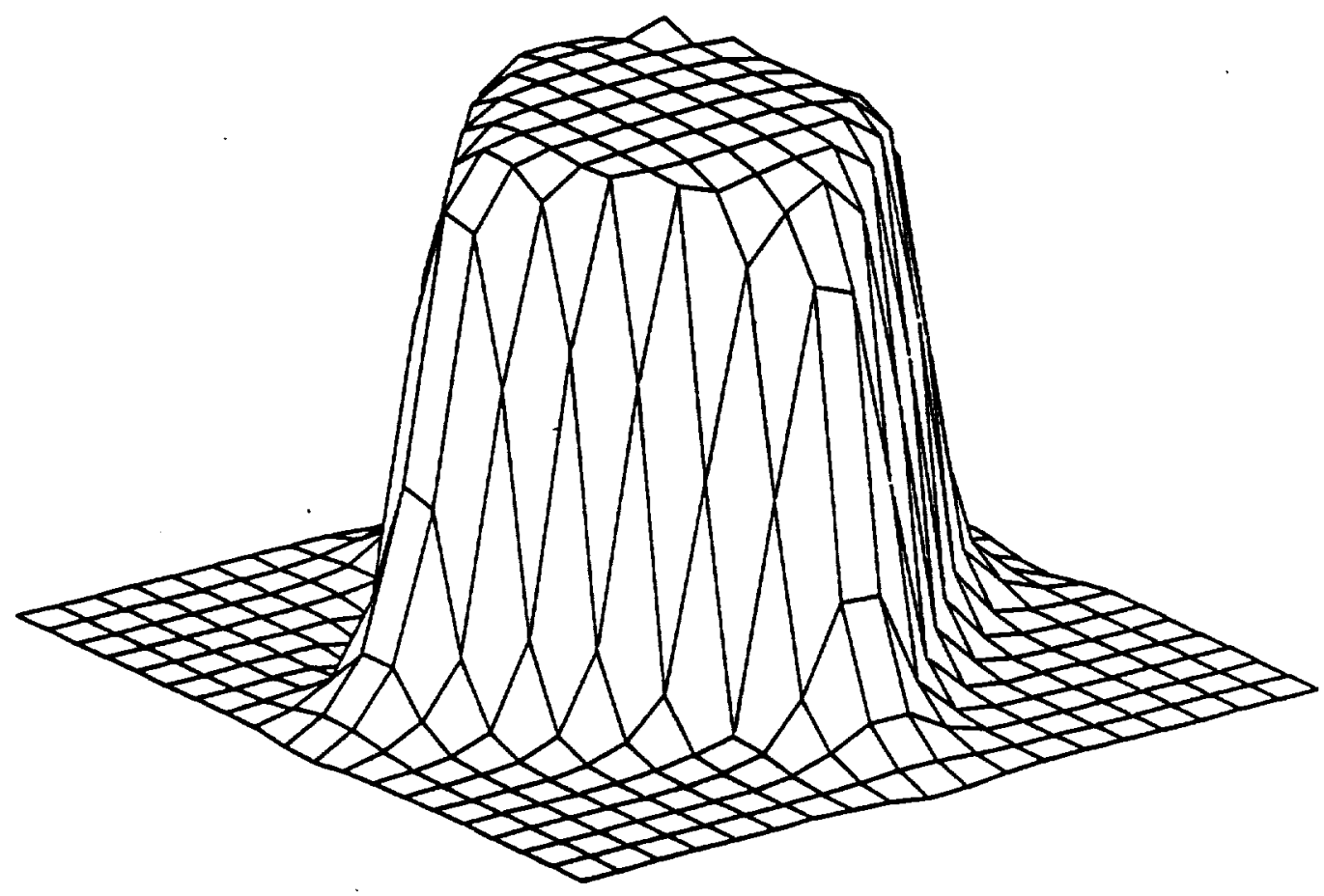

Figure 47: 4th order ENO, with artificial compression

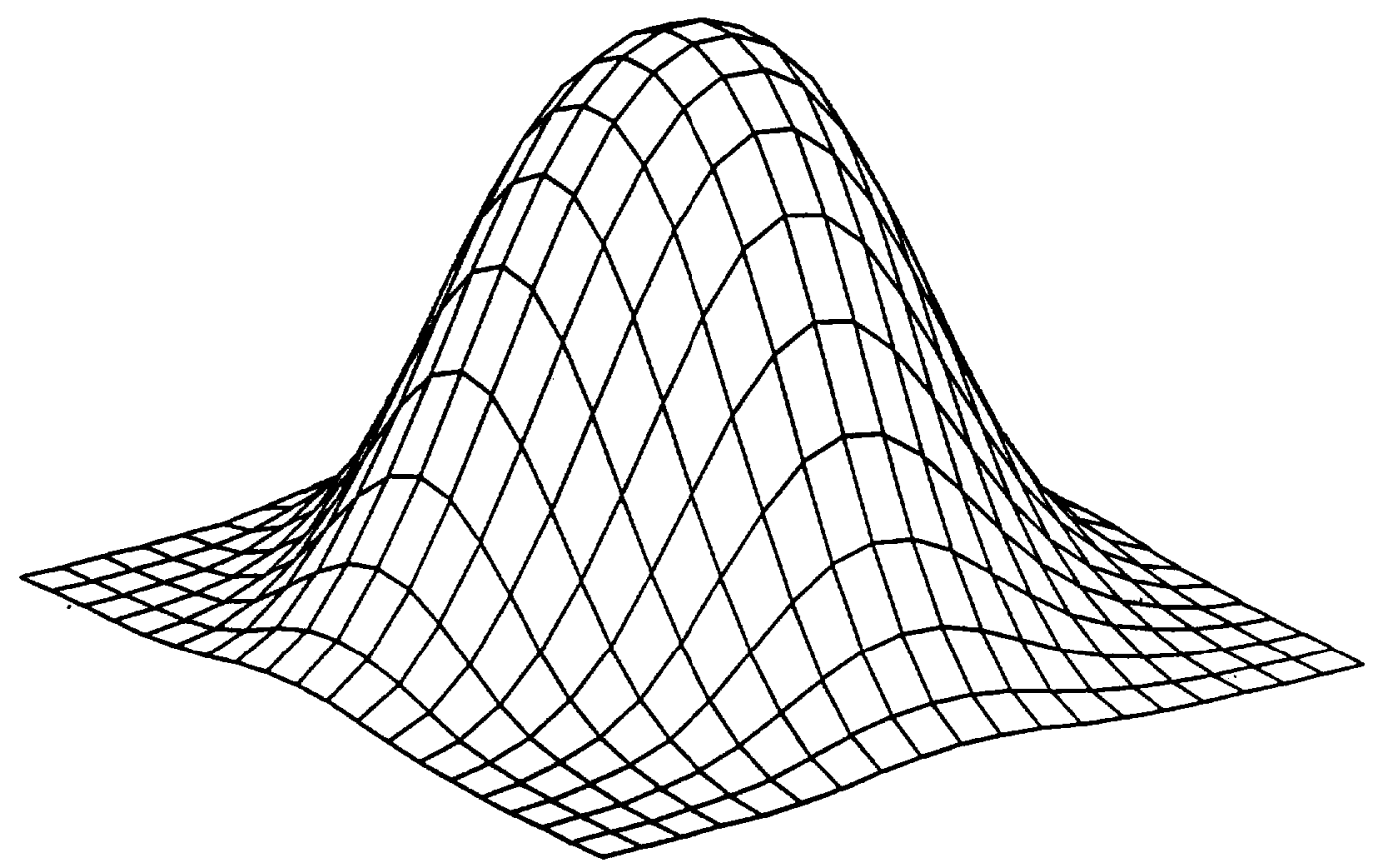

Figure 48: 2nd order ENO, without artificial compression 


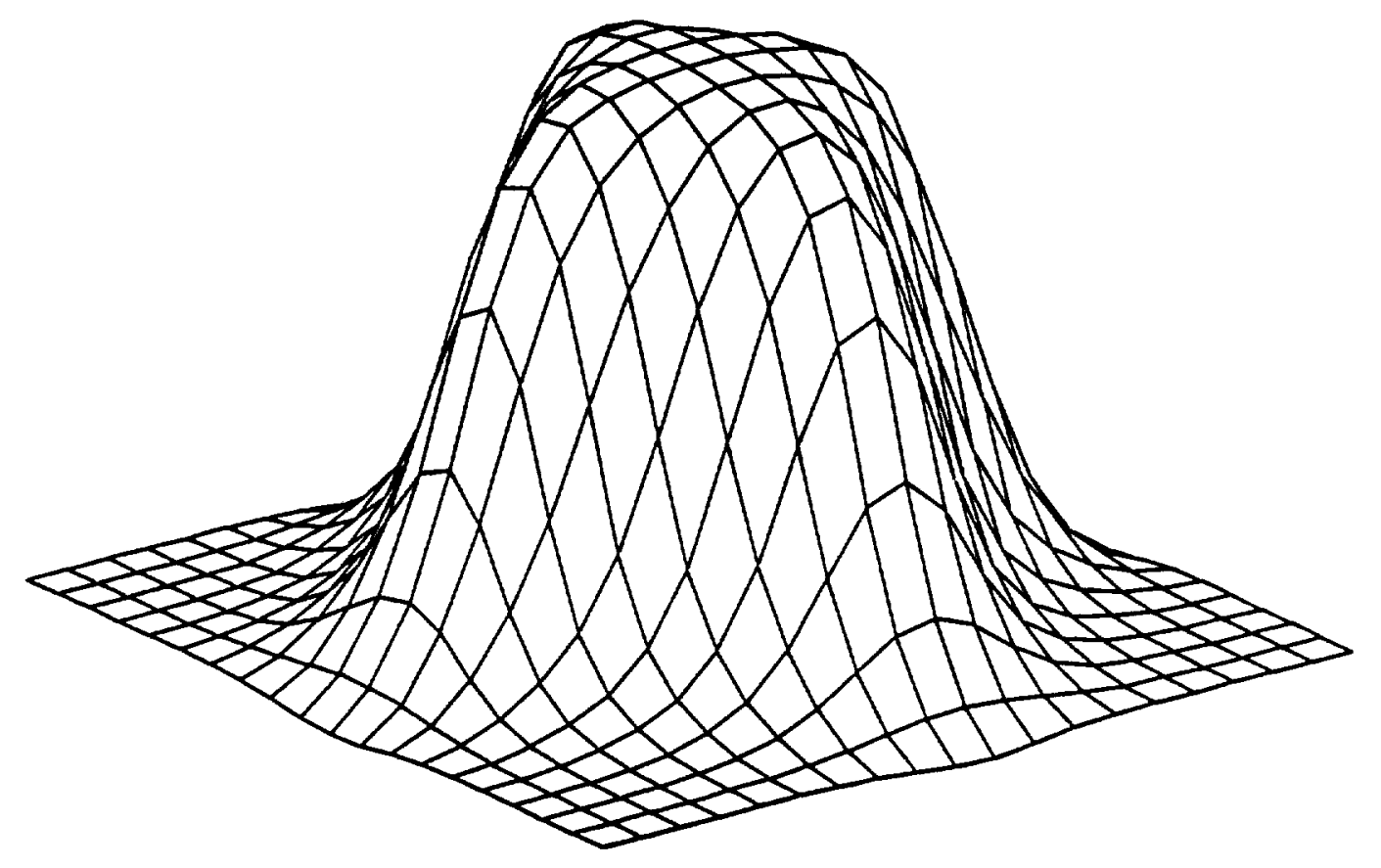

Figure 49: 3rd order ENO, without artificial compression

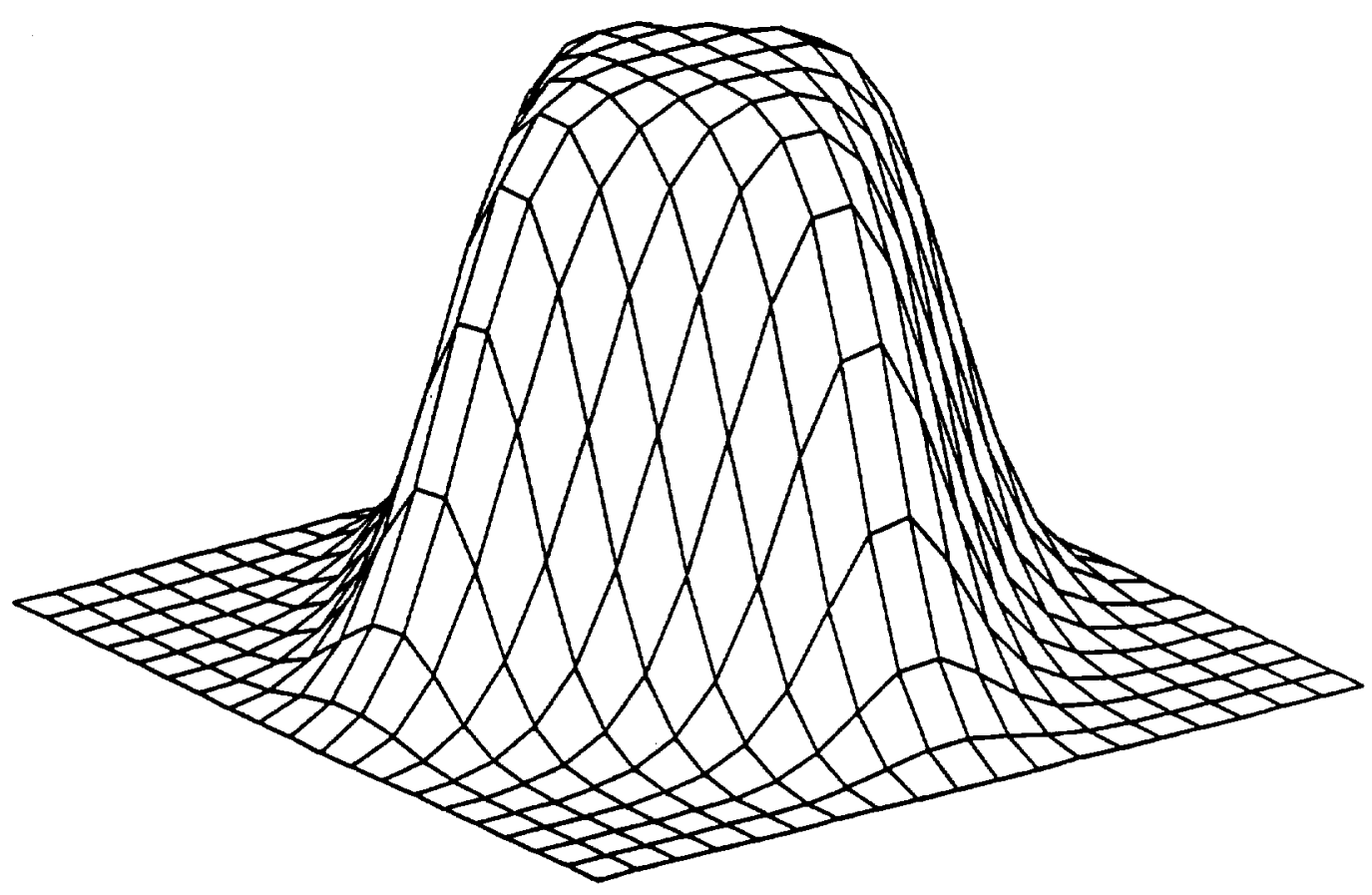

Figure 50: 4th order ENO, without artificial compression 
